\title{
HUMAN TRYPSINOGENS IN THE PANCREAS AND IN CANCER
}

\author{
Outi Itkonen \\ Department of Clinical Chemistry, University of Helsinki \\ and \\ Hospital District of Helsinki and Uusimaa - HUSLAB \\ Helsinki, Finland
}

\section{ACADEMIC DISSERTATION}

To be presented, with the permission of the Medical Faculty of the University of Helsinki, for public criticism in lecture room I, Meilahti Hospital, Haartmaninkatu 4,

Helsinki on September 12th at noon.

Helsinki 2008 
Supervised by

Professor Ulf-Håkan Stenman

Department of Clinical Chemistry, University of Helsinki, Finland

Revised by

Docent Jouko Lohi

Department of Pathology, University of Helsinki, Finland and

Docent Olli Saksela

Department of Dermatology, University of Helsinki, Finland

Examined by

Professor Kim Pettersson

Department of Biotechnology, University of Turku, Finland

ISBN 978-952-92-3963-4 (paperback)

ISBN 978-952-10-4732-9 (pdf)

http://ethesis.helsnki.fi

Yliopistopaino

Helsinki 2008 


\section{Contents}

Original publications $\quad 5$

$\begin{array}{ll}\text { Abbreviations } & 6\end{array}$

$\begin{array}{ll}\text { Abstract } & 7\end{array}$

Review of the literature $\quad 8$

Properties and biochemical characterization of human pancreatic trypsinogens 8

$\begin{array}{lr}\text { Extrapancreatic trypsinogen expression } & 12\end{array}$

Trypsinogen genes 13

Regulation of pancreatic trypsinogen gene expression and secretion 15

$\begin{array}{ll}\text { Regulation of gene expression } & 15\end{array}$

$\begin{array}{ll}\text { Regulation of secretion } & 16\end{array}$

$\begin{array}{ll}\text { Activation of trypsinogen to trypsin } & 17\end{array}$

$\begin{array}{ll}\text { Activation by enteropeptidase } & 17\end{array}$

$\begin{array}{ll}\text { Autoactivation } & 17\end{array}$

$\begin{array}{ll}\text { Activation by cathepsin B } & 17\end{array}$

$\begin{array}{ll}\text { Factors affecting trypsinogen activation } & 18\end{array}$

Trypsinogen and trypsin structure and mechanism of catalysis 20

$\begin{array}{ll}\text { Structure } & 20\end{array}$

Substrate binding $\quad 21$

Catalysis $\quad 22$

Functions of trypsins 23

Digestion of food 23

$\begin{array}{ll}\text { Activation of protease-activated receptors } & 23\end{array}$

Trypsinogens in cancer 25

Other functions of trypsins $\quad 26$

$\begin{array}{ll}\text { Trypsin inhibitors } & 27\end{array}$

$\alpha_{2}$-macroglobulin 28

$\begin{array}{ll}\alpha_{1} \text {-proteinase inhibitor } & 28\end{array}$

Inter- $\alpha$-inhibitors 28

PSTI or TATI $\quad 29$

Polyamines 31

Pancreatitis 31

Hereditary pancreatitis $\quad 32$

Clinical value of trypsinogen determinations $\quad 34$

Post-translational modification of proteins 36

Tyrosine $\boldsymbol{O}$-sulfation $\quad 36$

Effects of tyrosine-sulfation $\quad 37$

Post-translational modification of pancreatic trypsinogens 38

Aims of the present study

Materials and methods $\quad 40$

Samples, patients and cell lines (I- IV) $\quad 40$

$\begin{array}{ll}\text { Monoclonal antibodies (I) } & 40\end{array}$

Time-resolved immunofluorometric assays (I) $\quad 40$

Radioimmunoassays (I and II) 41

Characterization of trypsinogens by gel filtration chromatography (I and II) 42

Purification of trypsinogen (I, II and IV) 42 
Separation of trypsinogen isoenzymes by anion exchange

and RP HPLC (I, III and IV)

Activation of trypsinogen isoenzymes (I and II) 43

Alkylation and digestion of trypsinogens (IV) 43

Mass spectrometry (IV) $\quad 43$

Data analysis (IV) $\quad 44$

N-terminal sequence analysis (IV) $\quad 44$

Electrophoresis and immunoblotting (II and IV) 44

Statistical analysis (II) $\quad 44$

Results $\quad 45$

Immunoassays for trypsinogens/trypsin and concentrations in serum samples (I, III) 45

TAT and TATI concentrations in ovarian tumor cyst fluids (II) 47

Characterization of trypsinogen immunoreactivity by gel filtration (I, II) 47

Trypsinogens in anion exchange chromatography (I, III) 48

Purification of trypsinogen by reverse-phase HPLC (II, IV) 49

MS-analysis of trypsin and trypsinogen isoenzymes (IV) 49

Identification of the tryptic peptide with 80 Da mass addition (IV) 50

Identification of Tyr154 sulfation in trypsinogen-1 and -2 (IV) 50

Discussion $\quad 54$

TR-IFMAs for trypsinogen-1 and -2 54

Immunoreactive trypsinogen-1 and -2 in serum samples $\quad 54$

Immunoreactive trypsinogen-1 and -2 in cancer 56

$\begin{array}{ll}\text { Immunoreactive TATI in cancer } & 56\end{array}$

$\begin{array}{ll}\text { Characterization of pancreatic and extra-pancreatic trypsinogens } & 57\end{array}$

$\begin{array}{ll}\text { Investigation of Tyr154 modification } & 58\end{array}$

Aromatic interactions in proteins $\quad 59$

$\begin{array}{ll}\text { The effect of tyrosine sulfation on trypsin } & 59\end{array}$

$\begin{array}{ll}\text { Conclusions } & 61\end{array}$

$\begin{array}{ll}\text { Concluding remarks } & 62\end{array}$

$\begin{array}{ll}\text { Acknowledgements } & 64\end{array}$

References $\quad 66$ 


\section{Original publications}

This thesis is based on the following articles which are referred to in the text by their Roman numerals:

I Itkonen O, Koivunen E, Hurme M, Alfthan H, Schröder T and Stenman UH. Time-resolved immunofluorometric assays for trypsinogen- 1 and 2 in serum reveal preferential elevation of trypsinogen-2 in pancreatitis. J Lab Clin Med 115, 712-718 (1990).

II Koivunen E, Itkonen O, Halila H and Stenman UH. Cyst fluid of ovarian cancer patients contains high concentrations of trypsinogen-2. Cancer Res 50, 2375-2378 (1990).

III Itkonen O, Stenman UH, Osman S, Koivunen E, Halila H and Schröder T. Serum samples from pancreatectomized patients contain trypsinogen immunoreactivity. J Lab Clin Med 128, 98-102 (1996).

IV Itkonen O, Helin J, Saarinen J, Kalkkinen N, Ivanov KI, Stenman UH and Valmu L. Mass spectrometric detection of tyrosine sulfation in human pancreatic trypsinogens, but not in tumor-associated trypsinogen. FEBS J 275, 289-301 (2008).

Publication II was also included in the thesis entitled "Tumor-associated trypsinogen" by Ph.D, docent Erkki Koivunen, Department of Biological and Environmental Sciences, University of Helsinki, 1991. 


\section{Abbreviations}

AA amino acid

API $\quad \alpha_{1}$-proteinase inhibitor

CCK cholecystokinin

CF cystic fibrosis

CTSB cathepsin B

ECM extracellular matrix

ESI electrospray ionization

HP hereditary pancreatitis

IRT immunoreactive trypsinogen

LC liquid chromatography

$\mathrm{MAb}$ monoclonal antibody

MMP matrix metalloproteinase

MS mass spectrometry

MSMS tandem mass spectrometry

PTM post-translational modification

RIA radioimmunoassay

RP reverse-phase

TAP trypsinogen activation peptide

TAT tumor-associated trypsinogen

TATI tumor-associated trypsin inhibitor

TIMP tissue inhibitor of metalloproteinase

TPST tyrosylprotein sulfotransferase

TR-IFMA time-resolved immunofluorometric assay 


\section{Abstract}

Human pancreatic juice contains two major trypsinogen isoenzymes called trypsinogen-1 and -2 , or cationic and anionic trypsinogen, respectively. Trypsinogen isoenzymes are also expressed in various normal and malignant tissues. We aimed at developing monoclonal antibodies (MAbs) and time-resolved immunofluorometric assays recognizing human trypsinogen-1 and -2, respectively. Using these MAbs and assays we purified, characterized and quantitated trypsinogen isoenzymes in serum samples, ovarian cyst fluids and conditioned cell culture media.

In sera from healthy subjects and patients with extrapancreatic disease the concentration of trypsinogen-1 is higher than that of trypsinogen-2. However, in acute pancreatitis we found that the concentration of serum trypsinogen- 2 is 50 -fold higher than in controls, whereas the difference in trypsinogen-1 concentration is only 15 -fold. This suggested that trypsinogen- 2 could be used as a diagnostic marker for acute pancreatitis.

In human ovarian cyst fluids tumor-associated trypsinogen-2 (TAT-2) is the predominant isoenzyme. Most notably, in mucinous cyst fluids the levels of TAT-2 were higher in borderline and malignant than in benign cases. The increased levels in association with malignancy suggested that TAT could be involved in ovarian tumor dissemination and breakage of tissue barriers.
Serum samples from patients who had undergone pancreatoduodenectomy contained trypsinogen-2. Trypsinogen-1 was detected in only one of nine samples. These results suggested that the expression of trypsinogen is not restricted to the pancreas.

Determination of the isoenzyme pattern by ion exchange chromatography revealed isoelectric variants of trypsinogen isoenzymes in serum samples. Intact trypsinogen isoenzymes and tryptic and chymotryptic trypsinogen peptides were purified and characterized by mass spectrometry, Western blot analysis and $\mathrm{N}$-terminal sequencing. The results showed that pancreatic trypsinogen-1 and -2 are sulfated at tyrosine 154 (Tyr154), whereas TAT-2 from a colon carcinoma cell line is not. Tyr154 is located within the primary substrate binding pocket of trypsin, thus Tyr154 sulfation is likely to influence substrate binding. The previously known differences in charge, substrate specificity and inhibitor binding between pancreatic and tumorassociated trypsinogens are suggested to be caused by sulfation of Tyr154 in pancreatic trypsinogens. 


\section{Review of the literature}

The terms "enzyme" (from the Greek en - in and zume - yeast) and "trypsin" were first suggested by a german scientist, Wilhelm (Willy) Friedrich Kühne (1837-1900), when he found a substance in bovine pancreatic juice that degraded other biological substances. He proposed the term "enzyme" for non-organized ferments and "trypsin" for the enzyme that breaks down proteins. Kühne presented this paper in the $4^{\text {th }}$ February 1876 to the Heidelberger Naturhistorischen und Medizinische Verein, and was reprinted in 1976 (Kühne, 1976).

Trypsinogen was first characterized from cattle pancreatic extracts (Kunitz and Northrop, 1934, Northrop and Kunitz, 1932). In a study of Kunitz and Northrop (Kunitz and Northrop, 1935) bovine trypsinogen was shown to be activated either by enteropeptidase or active trypsin, indicating that the activation can be autocatalytic. Activation was shown to be $\mathrm{pH}$ dependent and maximal at $\mathrm{pH} 7.0$ to 8.0. The molecular masses of purified trypsin, trypsin complexed with a polypeptide inhibitor, and polypeptide trypsin inhibitor were reported to be $36500 \mathrm{Da}, 40000 \mathrm{Da}$ and $6000 \mathrm{Da}$, respectively. Trypsin was reversibly inhibited by the inhibitor. The activity, general properties and inhibition of various preparations of crystalline trypsin by the polypeptide inhibitor were also reported.

Before this study was started, tumor-associated trypsin inhibitor (TATI) had been isolated from urine of an ovarian cancer patient and shown to be identical to pancreatic secretory trypsin inhibitor (PSTI) (Huhtala et al., 1982). Elevated levels of TATI had been observed in urine from patients with ovarian, cervical and endometrial cancer. In search for a target protease for TATI, two trypsinogen isoenzymes were shown to be expressed in cyst fluid of mucinous ovarian tumors (Koivunen et al., 1989). The N-terminal amino acid sequences of these tumor-associated isoenzymes corresponded to those of pancreatic trypsinogen-1 and -2, respectively. However, the isoenzymes had different specificities for $p$-nitroanilide substrates, responded differently to various protease inhibitors and had different isoelectric points from those of trypsinogen-1 and -2 . Therefore, they were named tumorassociated trypsinogen-1 and -2 (TAT-1 and TAT-2) (Koivunen et al., 1989).

\section{Properties and biochemical characterization of human pancreatic trypsinogens}

Trypsinogen (Enzyme Commission (EC) number 3.4.21.4) was first reported to occur in human pancreatic juice by Haverback et al. (Haverback et al., 1960) and was among the first human enzymes to be purified and characterized (Buck et al., 1962). Human pancreatic juice contains two major trypsinogen isoenzymes called trypsinogen-1 and -2 , or cationic and anionic trypsinogen, respectively (Figarella et al., 1969, Keller and Allan, 1967, Rinderknecht and Geokas, 1972). Trypsinogen-2 is the most anionic protein in human pancreatic juice (Figarella et al., 1969). The proportion of trypsinogen-1 to trypsinogen-2 is about two to one in normal pancreatic juice (Figarella et al., 1969, Guy et al., 1978, Rinderknecht et al., 1979), and these two trypsinogens represent $19 \%$ of total proteins of pancreatic juice (Guy et al., 1978). Total trypsinogen concentration in human pancreatic juice is reported to be in the range of 4 to $40 \mu \mathrm{mol} / \mathrm{L}$ (Rinderknecht et al., 1979). A third, minor trypsinogen isoenzyme, called trypsinogen-3 or mesotrypsinogen, also occurs in human pancreatic juice (Rinderknecht et al., 1979, Rinderknecht et al., 1984, Scheele et al., 1981).

In some reports (Nyaruhucha et al., 1997, Scheele et al., 1981), but not in this thesis, the trypsinogen isoenzymes have been designated 


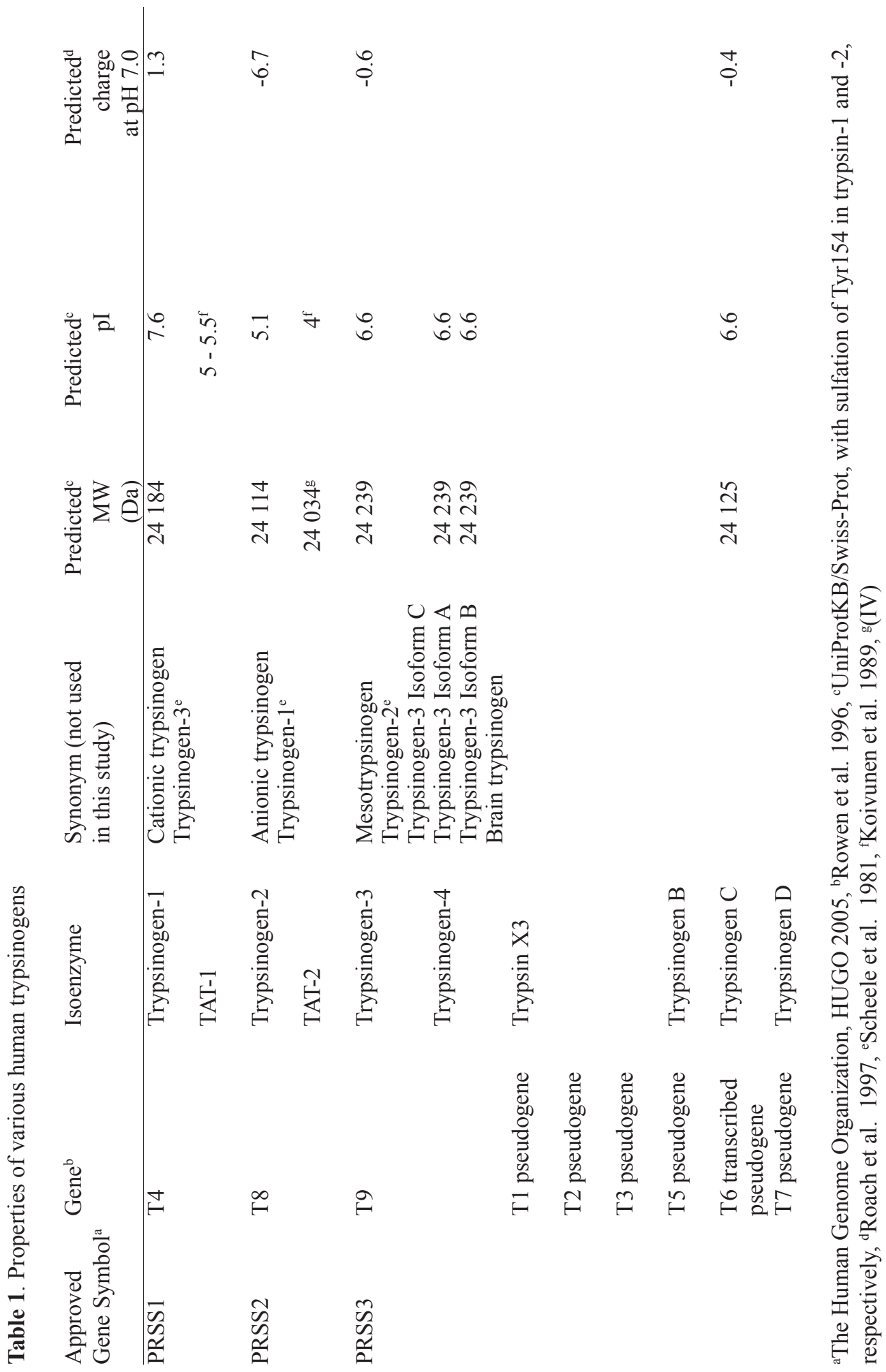


according to their isoelectric point, which causes confusion about the nomenclature of trypsinogens. Table 1 summarizes the properties and nomenclature of human trypsinogens and genes used throughout this study. UniProtKB/SwissProt numbering of amino acid residues (http://au.expasy.org/) is used unless otherwise stated.

Human trypsin-1 is inhibited completely by the Kunitz bovine inhibitor (BPTI), strongly by lima bean trypsin inhibitor (LBTI), moderately by the Bowman-Birk inhibitor and porcine Kazal inhibitor (PSTI) and only weakly by soybean trypsin inhibitor (SBTI). Chicken ovomucoid shows no inhibition whatsoever. Trypsin-2 is totally inhibited by BPTI, strongly by SBTI and LBTI, considerably more strongly inhibited than trypsin-1 by the Bowman-Birk inhibitor and porcine PSTI, and weakly by ovomucoid. Furthermore, human inter- $\alpha$-trypsin inhibitor (ITI) inhibits trypsin-2 more readily than trypsin-1. Human $\alpha_{1}$-proteinase inhibitor (API) completely inhibits both trypsin-1 and -2 (Figarella et al., 1975, Mallory and Travis, 1975). The optimal activity of trypsin isoenzymes is between 7.5 and 8.5 (Rinderknecht et al., 1984) and the proteolytic activities of trypsin- 1 and -2 have been found to be identical (Colomb et al., 1978). Generally, trypsin-2 is characterized to be less stable and undergo faster autolysis than trypsinogen-1 and it is more sensitive to inhibition by naturally occuring proteinase inhibitors (Colomb et al., 1978, Mallory and Travis, 1973, Rinderknecht and Geokas, 1972).

Trypsinogen-3 occurs at very low concentrations and represents probably $<0.5 \%$ of the proteins and $<5 \%$ of trypsinogens in normal human pancreatic juice (Nyaruhucha et al., 1997, Rinderknecht et al., 1984). Trypsinogen-3 resembles trypsinogen-1 and -2 in many properties, but it is not inhibited by either human pancreatic secretory trypsin inhibitor (PSTI) or other naturally occuring trypsin inhibitors (Nyaruhucha et al., 1997, Rinderknecht et al., 1984, Sahin-Tóth, 2005). In contrast, active trypsin-3 rapidly hydrolyzes and degrades the Kunitz-type trypsin inhibitor SBTI and Kazal-type inhibitor PSTI (Szmola et al., 2003). Furthermore, trypsin-3 was shown to selectively and rapidly cleave the Lys 10-Thr11 peptide bond of API. Subsequent mutagenesis studies revealed that trypsin-3 exhibits an unusually restricted $S$ ' subsite specificity but can efficiently digest Lys/ Arg - Ser/Thr peptide bonds in polypeptide substrates (Szepessy and Sahin-Tóth, 2006).

The stability of trypsin-3 resembles that of trypsin-2, its $\mathrm{pH}$ optimum is at 8.2 and it needs calcium for full enzymatic activity (Rinderknecht et al., 1984). In contrast to the conserved features of trypsinogen isoenzymes in various species, an arginine in stead of glycine is present at residue 198 in trypsinogen-3 (Roach et al., 1997). This residue was shown by $\mathrm{x}$-ray chrystallography (Katona et al., 2002) to be located in the substrate binding pocket of trypsinogen and was suggested to be the structural basis for the nearly total resistance of trypsin-3 to natural trypsin inhibitors (Nyaruhucha et al., 1997). This was confirmed by studies on trypsinogen-3 mutant Arg 198Gly (Szmola et al., 2003). Paradoxally, the Arg 198 substitution also renders trypsin-3 more resistant to autocatalytic degradation (Szmola et al., 2003).

Pancreatic trypsinogen- $1,-2$ and -3 cDNAs contain 741 bp of coding region, which translates to a single polypeptide chain with 247 amino acids (AA) (Table 2). The three preproenzymes share about $87 \%$ homology and all the typical sequence features of a trypsinogen: a fifteen AA signal sequence, an eight AA activation peptide, the catalytic triad comprising residues His63, Asp107 and Ser200, the four key pocket specificity residues Asp194, Gln197, Gly217 and Gly227, and the six cysteine residues needed for the conserved disulfide bridges (48-64, 171-185, and 196-220). In addition to these, trypsinogen-2 contains a disulfide bridge at 30-160 and trypsinogen-1 and -3 contain two additional ones at 30-160 and 139-206. Interestingly, all other known trypsinogens from higher vertebrates contain six disulfide 


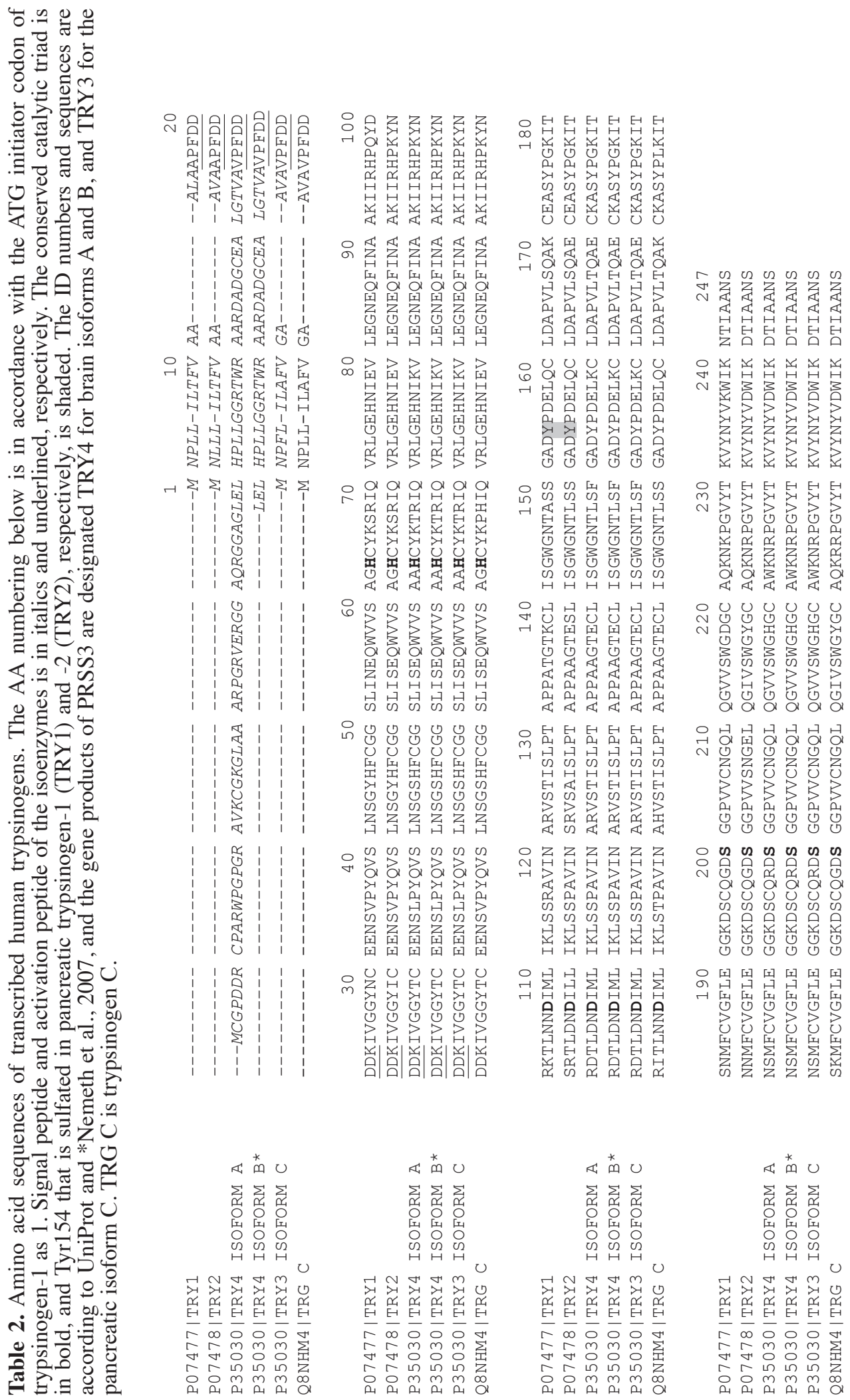


bonds (Kenesi et al., 2003). The activation peptide contains a cluster of anionic residues, namely four aspartates preceding a positively charged lysine residue, a conserved feature of mammalian trypsinogens (Chen and Feréc, 2000c, Roach et al., 1997).

Human trypsinogen-1 and trypsin-1 may occur in single- and double-chain forms. Singlechain trypsin is called $\beta$-trypsin. The doublechain form is produced by autocatalytic cleavage of the Arg122-Val123 peptide bond of $\beta$-trypsin, the two chains being held together by a disulfide bond. Studies using recombinant human trypsinogen-1 reveal these two forms to be functionally identical. However, cleavage of the Arg122-Val123 bond in trypsinogen-1 inhibits trypsinogen-1 autoactivation and this may be one of the protective mechanisms of premature trypsinogen activation in the pancreas (Kukor et al., 2002b).

Both trypsinogen-1 and -2 contain two calcium binding sites (Abita et al., 1969, Colomb and Figarella, 1979). The so called high affinity calcium ion binding loop (Glu75 - Glu85) common to trypsinogen and active trypsin maintains the enzyme in its active form and simultaneously protects it from autodegradation (Abita et al., 1969, Bode and Schwager, 1975a, Bode and Schwager, 1975b, Szmola and Sahin-Tóth, 2007). The second calcium binding site located in the region of four Asp residues of the activation peptide is present in the zymogen only. The balance between trypsin activation and degradation is regulated by $\mathrm{Ca}^{2+}$ concentration. At low $\mathrm{Ca}^{2+}$ concentrations chymotrypsin $\mathrm{C}$ (denoted enzyme Y by Rinderknecht (Rinderknecht et al., 1988)) cleaves with high selectivity the Leu81-Glu82 bond within the $\mathrm{Ca}^{2+}$ binding loop of trypsin-1 resulting in rapid degradation and loss of trypsin activity by subsequent tryptic cleavage of the autolysis site Arg122Val123. Increasing the $\mathrm{Ca}^{2+}$ concentration progressively inhibits the degradation of trypsin- 1 by chymotrypsin C. At $1 \mathrm{mmol} / \mathrm{L}$ $\mathrm{Ca}^{2+}$ chymotrypsin $\mathrm{C}$ mediated cleavage of the Leu81-Glu82 is essentially completely inhibited by stabilizing the $\mathrm{Ca}^{2+}$ binding loop.
Thus, at the high $\mathrm{Ca}^{2+}$ concentration in the duodenum chymotrypsinogen $\mathrm{C}$ facilitates trypsinogen autoactivation (see below), whereas at low $\mathrm{Ca}^{2+}$ concentration in the lower small intestines chymotrypsin $\mathrm{C}$ promotes trypsin degradation (Szmola and Sahin-Tóth, 2007).

Detailed analysis of the evolution of trypsinogen activation peptide demonstrated that the Asp-Asp-Asp-Asp-Lys sequence in mammalian trypsinogens has evolved to inhibit autoactivation and enhance cleavage by enteropeptidase (Chen et al., 2003a). Under physiological conditions in the pancreatic juice (pH 8 and $1 \mathrm{mmol} / \mathrm{L} \mathrm{Ca}^{2+}$ ) this calcium site is probably saturated in trypsinogen- 1 but not in trypsinogen-2. This facilitates trypsinogen-1 autoactivation. The $\mathrm{Ca}^{2+}$ binding site common to trypsin and trypsinogen has been reported to have pKa $\left(\mathrm{Ca}^{2+}\right)$ values of 2.8 and 3.4 and the $\mathrm{Ca}^{2+}$ binding site present in trypsinogen only has $\mathrm{pKa}\left(\mathrm{Ca}^{2+}\right)$ values of 3.3 and 2.7 for trypsinogen-1 and -2 , respectively (Colomb and Figarella, 1979).

\section{Extrapancreatic trypsinogen expression}

Extrapancreatic expression of human trypsin immunoreactivity or mRNA has been detected in the Paneth cells of the gastrointestinal mucosa (Bohe et al., 1986, Ghosh et al., 2002), in the brain (Wiegand et al., 1993), male genital tract (Paju et al., 2000), epithelial cells of the skin, esophagus, stomach, small intestine, lung, kidney, liver, and extrahepatic bile duct, and splenic macrophages, monocytes and lymphocytes, the nerve cells of hippocampus and cerebral cortex in the brain (Kawano et al., 1997, Koshikawa et al., 1998), colonic mucosa (Cottrell et al., 2004), vascular endothelial cells (Koshikawa et al., 1997), cerebrospinal fluid (Critchley et al., 2000), synovial cells and synovial fluid (Stenman et al., 2005), tracheal aspirate fluid and lung tissue (Cederqvist et al., 2003), in human bronchoalveolar lavage fluid (Prikk et al., 2001), and in human milk (Borulf et al., 1987). 
Koivunen et al. showed that two trypsinogen isoenzymes are expressed in cyst fluid of mucinous ovarian tumors (Koivunen et al., 1989). The N-terminal amino acid sequences of these tumor-associated isoenzymes corresponded to those of pancreatic trypsinogen-1 and -2 , respectively. However, the isoenzymes had different specificities for $p$-nitroanilide substrates, responded differently to various protease inhibitors and had isoelectric points different from those of pancreatic trypsinogen-1 and -2 . Therefore, they were named tumor-associated trypsinogen-1 and trypsinogen-2 (TAT-1 and TAT-2) (Koivunen et al., 1989). TAT-1 and TAT-2 were found to be less anionic than trypsinogen-1 and -2 when separated by ion exchange chromatography (Koivunen et al., 1991b). However, the nucleotide sequence of TAT-2 and pancreatic trypsin-2 is identical (Sorsa et al., 1997). Since then, trypsinogen immunoreactivity or mRNA has been detected in various cancers like stomach, pancreas, ovary, lung, bladder, esophagus, bile duct, and colon cancers, and carcinoma cell lines (Bernard-Perrone et al., 1998, Bjartell et al., 2005, Hirahara et al., 1995, Hotakainen et al., 2006, Kato et al., 1998, Kawano et al., 1997, Kawano et al., 1997, Koivunen et al., 1991b, Koshikawa et al., 1992, Koshikawa et al., 1994, Miyagi et al., 1995, Miyata et al., 1999, Nyberg et al., 2002, Ohta et al., 1998, Oyama et al., 2000, Paju et al., 2004, Stenman et al., 2003, Terada et al., 1995, Terada et al., 1997, Williams et al., 2001, Yamashita et al., 2003).

Tumor-associated trypsins as well as other proteinases have been recognized as significant factors in cancer progression and metastatic processes such as cellular invasion, degradation of extra-cellular matrix proteins, angiogenesis and tissue remodeling as reviewed in (Nyberg et al., 2006). Extracellular proteolysis in cancer can be initiated by the urokinase plasminogen activator (UPA), uPA receptor (UPAR) and plasminogen, which in turn activates latent matrix metalloproteinases (MMPs). MMPs are secreted or transmembrane proteins that are capable of digesting extracellular matrix (ECM) and basement membrane components under physiological conditions. MMPs are associated with metastatic phenotype of malignant cells and they are considered to be the major functional contributors to metastatic processes (Chambers and Matrisian, 1997). Trypsin, too, degrades many ECM components (Koivunen et al., 1991a, Koshikawa et al., 1992, Moilanen et al., 2003, Stenman et al., 2005) but it is also a potent activator of several MMPs (Imai et al., 1995, Koivunen et al., 1989, Moilanen et al., 2003, Nyberg et al., 2002, Paju et al., 2001b, Sorsa et al., 1997, Umenishi et al., 1990) and could thus initiate proteinase cascades and participate in modulation of tumor cell behavior.

Tumor-associated trypsin expression has been shown to correlate with malignancy in various cancers and of the four known trypsin isoforms, TAT-2 seems to be most common in tumors (Hirahara et al., 1998, Ichikawa et al., 2000, Kato et al., 1998, Miyata et al., 1999, Nyberg et al., 2002, Paju et al., 2004, Yamamoto et al., 2001, Yamamoto et al., 2003). On the other hand, in microarray analysis trypsinogen IVb and trypsinogen $\mathrm{C}$ (see below) gene expression have been shown to be up-regulated in nonsmall cell lung cancer metastasis (Diederichs et al., 2004).

\section{Trypsinogen genes}

Trypsinogens are encoded by the protease, serine (PRSS) genes. Eight trypsinogen genes (denoted T1 to T8) divided into two clusters, have been located within the $\beta$ T-cell receptor (TCR) locus on chromosome region $7 \mathrm{q} 35$ (Rowen et al., 1996). Of these, five (T4 to T8) are tandemly arrayed 10-kb locus-specific repeats at the 3 ' prime end of the $\beta$ TCR locus. These repeats exhibit 90 to $91 \%$ overall nucleotide similarity, and embedded within each is a trypsinogen gene. Each gene contains five exons that span approximately $3.6 \mathrm{~kb}$. In addition, there are two pseudo trypsinogen genes and one relic trypsinogen gene at the 5' prime end of the $\beta$ TCR locus (T1 to T3), all in inverted transcriptional orientation. Earlier, T4 and T8, also known as PRSS1 and $P R S S 2$, have been identified as the cDNAs for 
trypsinogen-1 and -2, respectively (Emi et al., 1986) (Table 1).

Polymerase chain reaction (PCR) analyses of pancreas, thymus and liver suggest that the third apparently functional trypsinogen gene (T6) in the $\beta$ TCR locus may be expressed in minute amounts in the thymus (Rowen et al., 1996). This is further supported by expressed sequence tags (ESTs) AA295419 and AA295738. Comparison of the threedimensional structures of T4, T6 and T8 gene products suggests that the catalytic triad of His63, Asp107 and Ser200 and the nature of substrate binding pocket are highly conserved. However, the protein product of T6 might interact differentially with other proteins, as suggested by variations in surface charge and shape distributions (Chen and Feréc, 2000c, Rowen et al., 1996). T6 may represent a transition state between a functioning duplicated gene and a nonfunctional pseudogene (Chen et al., 2001). The protein product of T6 (trypsinogen C) has so far not been identified.

The $\mathrm{T} 1$ gene was described to be a pseudogene (Rowen et al., 1996), but it could be a proteincoding gene according to Entrez database used by the National Center for Biotechnology Information (www.ncbi.nlm.nih.gov). The protein product of $\mathrm{T} 1$ gene (trypsin X3) has not yet been identified. T2 and T3, T5 and T7 have been identified as nonfunctional pseudogenes (Rowen et al., 1996).

A third trypsinogen cDNA and its product has independently been identified as trypsinogen-3 and -4 (Nyaruhucha et al., 1997, Tani et al., 1990, Wiegand et al., 1993). The chromosomal location of the gene encoding this isoenzyme was located to chromosome region 9p13 (Rowen et al., 1996). This gene (T9 or PRSS3) is formed by segmental duplications originating from chromosomes $7 \mathrm{q} 35$ and 11q24 and it has two distinct promoters derived from each of the originating chromosomes. Thus, the transcripts of PRSS3 display two variants of exon 1 and share exons 2 to 5 which encode the active protease (Rowen et al., 2005).
The gene coding for functional trypsinogen-3 spans about $3.6 \mathrm{~kb}$, it is duplicatively transferred from chromosome 7 and expressed in the pancreas (isoform C). The PRSS3 variant coding for brain trypsinogen-4 spans $48.6 \mathrm{~kb}$ and it is a hybrid of an exon copied from chromosome 11 and four exons copied from chromosome 7. Allelic variants of this splice form (isoform A) are called $a$ and $b$. Another splice form named isoform B includes an additional exon derived from chromosome 7 after the chromosome 11 derived exon. Translation initiation site of the isoform $\mathrm{A} a / b$ splice forms is thought to be in the first exon derived from chromosome 11 starting from AUG translation initiation codon and coding for a 72-residue leader peptide (Nemeth et al., 2007). Translation of the B isoform is predicted to start in its second exon coding for a 28-residue leader peptide. This leader peptide has leucine as an initiator amino acid, and it is encoded starting from a CUG initiation codon (Nemeth et al., 2007).

The leading exon determines the pathway of the protein. In the pancreas, a secretory pancreatic trypsinogen-3 is produced. However, a cytosolic trypsinogen- 4 missing the typical leader peptide of secreted proteins is produced in the brain (Wiegand et al., 1993), epithelial cells of the colon, prostate, lung (Cottrell et al., 2004), several cancer cell lines, uterus, heart, hypothalamus and cerebellar cortex (Rowen et al., 2005). On the other hand, trypsinogen-4 a/b contains four Arg-X-XArg furin cleavage-recognition sites (Molloy et al., 1992) and trypsinogen-4 (a-form) has been detected in vesicles in transfected cell lines and in epithelial cells, supporting the possibility of an alternative secretion pathway (Cottrell et al., 2004, Wiegand et al., 1993).

No traces of trypsinogen-4 isoform A were found in the human brain by sequencing trypsinogen-4 samples isolated from human brain following a short post mortem delay (Nemeth et al., 2007). Instead, only isoform B of trypsinogen-4 was identified. When trypsinogen-4 is expressed in the U87 human glioblastoma cell line, the relative expression 
level of isoform A using an AUG initiation codon was elevated as compared to the expression level of isoform B with the CUG initiation codon. These results suggest that CUG as the initiation codon may control the expression level of the protein. Thus, directing the incorporation of $\mathrm{N}$-terminal leucine rather than methionine into isoform B of trypsinogen-4 is suggested to keep trypsinogen-4 expression in the brain at a relatively low level (Nemeth et al., 2007). The AA sequences of human trypsinogen-1, -2 , -3 isoform $\mathrm{C},-4$ isoform $\mathrm{A},-4$ isoform $\mathrm{B}$ and trypsinogen $\mathrm{C}$ are presented in Table 2 .

\section{Regulation of pancreatic trypsinogen gene expression and secretion}

\section{Regulation of gene expression}

The PTF1 complex. The exocrine pancreas is formed by acinar cells that synthesize and secrete digestive enzymes. Pancreas-specific expression of the about twenty acinar secretory enzymes at very high levels is controlled largely by the pancreatic transcription factor 1 (PTF1) complex (Cockell et al., 1989). PTF1 is an unusual heterotrimeric transcription factor. It contains a class A basic helix-loophelix (bHLH) protein (p75), which is required for import of the transcription factor into the nucleus (Sommer et al., 1991) and two DNA-binding subunits previously called p48 and p64 (Roux et al., 1989). The P48/PTF1a subunit is an exocrine pancreas-specific bHLH protein (Krapp et al., 1996) that is unable to bind to DNA alone and has to oligomerize with p64 in order to do so (Krapp et al., 1996, Sommer et al., 1991). P64 has been shown to be mammalian suppressor of hairless (RBP-J) or its paraloque, RBP-L. In the adult pancreas, RBP-L provides the strong transcriptional activity of the PTF1 complex that drives the high-level expression of the digestive enzyme genes (Beres et al., 2006).

PTF1 binds to DNA in the 5' promoter region of acinar digestive enzyme genes (Cockell et al., 1989, Rose et al., 2001). The binding sites of the PTF1 complex are bipartite with an E-box (preferably CACCTG) and a TCbox (TTTCCCA) spaced one or two helical turns apart, center to center (Cockell et al., 1989, Rose et al., 2001). Whereas an E-box is sufficient to bind the P48-bHLH heterodimer and a TC-box is sufficient to bind the P48RBP heterodimer, the trimeric complex requires both binding sites. Moreover, the binding of the trimeric complex is highly cooperative and can be much greater than the sum of the individual bindings, i.e. E-box for the bHLH and the TC-box for the RBP. This means that the formation of the trimeric PTF1 complex creates a synergistic dependence on the presence of both DNA sites spaced appropriately (Beres et al., 2006).

Cholecystokinin. It is known that acinar cell growth, energy production, gene expression and protein synthesis are also regulated by secretagogues. Cholecystokinin (CCK) increases the synthesis of pancreatic proteases including trypsinogen-1 by a prolonged effect on mRNA levels in the rat (Rosewicz et al., 1989). The binding of CCK to its cell surface receptor activates the mitogenactivated protein kinase (MAPK) cascades by various pathways like the extracellular signalregulated kinases 1 and 2 (ERK1/2) cascade, the Jun N-terminal kinase (JNK) cascade and the $\mathrm{p} 38$ MAPK cascade, resulting in activation of gene transcription, protein translation, metabolism and functions of the cytoskeleton (Duan et al., 1995, Williams, 2001). CCK also activates the phosphoinositide 3-kinase - mammalian target of rapamycin - 70-kDa ribosomal protein S6 kinase (PI3K - mTOR - P70 ${ }^{\mathrm{S} 6 \mathrm{~K}}$ ) signalling pathway, that primarily regulates protein synthesis at mRNA level, but is also required for mitogenesis (Crozier et al., 2006, Williams, 2001). Furthermore, using rat pancreatic acinar cells, CCK has been shown to activate the transcription factor nuclear factor- $\kappa \mathrm{B}(\mathrm{NF}-\kappa \mathrm{B})$, which is required for the production of chemokines and cytokines by pancreatic acinar cells (Han et al., 2001). Thus, CCK also affects acinar cell gene transcription, protein synthesis and growth in several ways. 
Dietary components. Dietary components can regulate digestive enzyme transcription, as with each meal the pancreas must synthesize new digestive enzymes to replace those secreted. In humans, enteral but not parenteral (intravenous) feeding has been demonstrated to increase trypsinogen synthesis (O'Keefe et al., 2006) and serum trypsinogen-1 immunoreactivity transiently (Florholmen et al., 1984a). Furthermore, dietary amino acids, especially branched chain AAs, have been shown to regulate pancreatic protein synthesis at the translation/initiation level, independently of hormonal and neuronal input, by phosphorylation of eukaryotic initiation factor eIF4E, its binding protein 4E-BP1, the ribosomal protein $\mathrm{S} 6$ kinase, and the formation of the eIF4F complex (Sans et al., 2006).

Other factors. Chemically modified tetracyclines (CMTs) and doxycycline (DOXY), which are chemical inhibitors of MMPs, have been shown to down-regulate the expression of TAT- 2 mRNA and TAT- 2 secretion by human COLO-205 cells (Lukkonen et al., 2000). Utilizing cDNA approach to identify genes differentially regulated during pancreatic regeneration after partial pancreatectomy in mice, the mitogenic $\operatorname{Reg} 3 \beta$ protein was shown to be induced in the acinar pancreas. Under these conditions, there was a 1.53-fold change in trypsin-2 gene expression (De Leon et al., 2006).

\section{Regulation of secretion}

The newly synthesized zymogens are segregated into condensing vacuoles, which undergo maturation to zymogen granules and are stored in the apical pole of the acinar cell. The secretion of pancreatic digestive enzymes is controlled physiologically by the vagal nerve, whose postganglionic neurons release acetylcholine, and by gastrointestinal hormones such as CCK, serotonin (Owyang and Logsdon, 2004), secretin, vasoactive intestinal polypeptide (VIP) and neuromedin C (Williams, 2001). Meal-stimulated CCK release from the intestinal mucosa represents the major physiological pathway for trypsinogen secretion (Owyang, 1996). The action of acetylcholine and CCK is mediated by G-protein coupled receptors on acinar cells (Williams, 2001). Human acinar cells contain mostly CCK2 receptors and proteinase-activated receptor-2 (PAR-2). Also CCK1 receptors have been detected in human pancreatic acinar cells (Galindo et al., 2005). CCK2 receptors bind both CCK and gastrin with high affinity (Owyang and Logsdon, 2004) whereas PAR-2 is activated by trypsin (Nguyen et al., 1999). It has also been suggested, that CCK stimulation of human pancreatic cells is regulated by an indirect mechanism of stimulation of afferent neurons (Ji et al., 2001). The neurohormonal regulation of pancreatic exocrine secretion is reviewed in (Nathan and Liddle, 2002).

Intracellular $\mathrm{Ca}^{2+}$ is considered to be the primary signaling factor in acinar cells as it triggers the fusion of zymogen granules with the apical plasma membrane and exocytosis. Levels of intracellular $\mathrm{Ca}^{2+}$ are modulated by activated $\mathrm{G}$ proteins and other signalling molecules like phospholipase $\mathrm{C} \beta$, inositol triphosphate $\left(\mathrm{IP}_{3}\right)$, diacylglycerol, cyclic ADP ribose, nicotinic acid adenine dinucleotide phosphate, and by intracellular and plasma membrane $\mathrm{Ca}^{2+}$ ATPase pumps, and plasma membrane $\mathrm{Ca}^{2+}$ channels (Petersen, 2004, Turvey et al., 2005, Williams, 2006). The endoplasmic reticulum has been established as the primary site for $\mathrm{Ca}^{2+}$ release, but the acidic lysosomal-like compartment and mitochondria are also involved (Williams, 2006). The packaging, movement, and fusion of zymogen granules to the apical membrane of acinar cells is affected by several zymogen granule membrane proteins like the SNARE proteins, small $\mathrm{G}$ proteins of the Rab family, cyclic AMP, diacylglycerol, and actin filaments (Wasle and Edwardson, 2002, Williams, 2006). 


\section{Activation of trypsinogen to trypsin}

\section{Activation by enteropeptidase}

The intrinsic catalytic activity of trypsinogen is $\sim 10^{8}$-fold lower than that of trypsin (Pasternak et al., 1998). Trypsinogens are activated into trypsins on cleavage of the eight-AA activation peptide by enteropeptidase (EC 3.4.21.9, also known as enterokinase) when they enter the duodenum. Enteropeptidase is a membranebound serine protease located in the enterocytes and goblet cells of the brush-border and glycocalyx of the duodenum and the proximal $15 \mathrm{~cm}$ of jejunum (Hermon-Taylor et al., 1977, Imamura and Kitamoto, 2003). It is an $\mathrm{N}$-glycosylated, disulfide-linked heterodimer that is derived from a single-chain precursor. The heavy chain anchors enteropeptidase in the intestinal brush border membrane and the light chain is the catalytic subunit, which has the same mechanism of action as trypsin and chymotrypsin (Kitamoto et al., 1994, Light and Janska, 1989). Enteropeptidase itself has been proposed to be activated by a trypsin- and chymotrypsin-like protease called duodenase that cleaves a Lys-Ile bond in the amino terminus of the enteropeptidase light chain (Zamolodchikova et al., 2000).

The specificity of enteropeptidase for cleavage after Lys has been proposed to be consistent with the presence of Asp981 at the base and two Gly residues at the sides of the specificity pocket that binds the $\mathrm{P} 1$ substrate residue. The Arg-Arg-Arg-Lys sequence at residues 886 to 889 may interact directly with the Asp residues in positions P2 to P5 of trypsinogen substrates (Kitamoto et al., 1994). However, it has been shown by site-directed mutagenesis of human trypsinogen-1 that the four Asp residues in the activation peptide are not required for enteropeptidase recognition and they confer only a modest catalytic improvement of enteropeptidase-mediated trypsinogen activation in humans (Nemoda and SahinTóth, 2005). Human trypsinogen-1 and -2 are activated by enterokinase at the same rate (Colomb and Figarella, 1979).

\section{Autoactivation}

Trypsinogen- 1 and -2 can also be autoactivated by either human trypsin at the same rate, but the affinity of both trypsin- 1 and -2 is higher for trypsinogen-1 than for trypsinogen-2. In presence of $1 \mathrm{mmol} / \mathrm{L}$ calcium at $\mathrm{pH}$ 5.6 the autoactivation of trypsinogen-1 becomes predominant compared to enterokinase activation. This suggests that under physiological conditions in the duodenum, enteropeptidase is the starter of trypsinogen activation but the predominant subsequent mechanism becomes trypsinogen autoactivation (Colomb and Figarella, 1979, Nemoda and Sahin-Tóth, 2005). Contrarily to trypsinogen-1 and -2, pancreatic trypsinogen-3 can neither autoactivate nor activate or degrade other pancreatic zymogens (Sahin-Tóth, 2005, Szilagyi et al., 2001, Szmola et al., 2003).

\section{Activation by cathepsin B}

Trypsinogen can be activated by lysosomal cysteine protease cathepsin B (CTSB) in vitro (Figarella et al., 1988) and in vivo in a mouse model (Halangk et al., 2000). There are several reports to support this activation mechanism in humans, too. Cathepsin B is abundantly present also in the human pancreatic secretory compartment and it is secreted together with trypsinogen into pancreatic juice (Kukor et al., 2002a). CTSB activates human trypsinogen-1 with trypsin yield of about $30 \%$ of that produced by enterokinase in vitro (Lindkvist et al., 2006). CTSB has been shown to activate recombinant trypsinogen-3 more readily than trypsinogen-1 or -2 at $\mathrm{pH} 4.0$ (Szmola et al., 2003). This suggests that the premature intracellular activation of trypsinogen in acute pancreatitis might be initiated by the action of CTSB on trypsinogen-3 leading to degradation of PSTI, which contributes to the development of human pancreatitis. Furthermore, the Lys26Val mutation in the CTSB propeptide region is associated with tropical calcific pancreatitis (TCP) (Mahurkar et al., 2006). This mutation could affect CTSB 


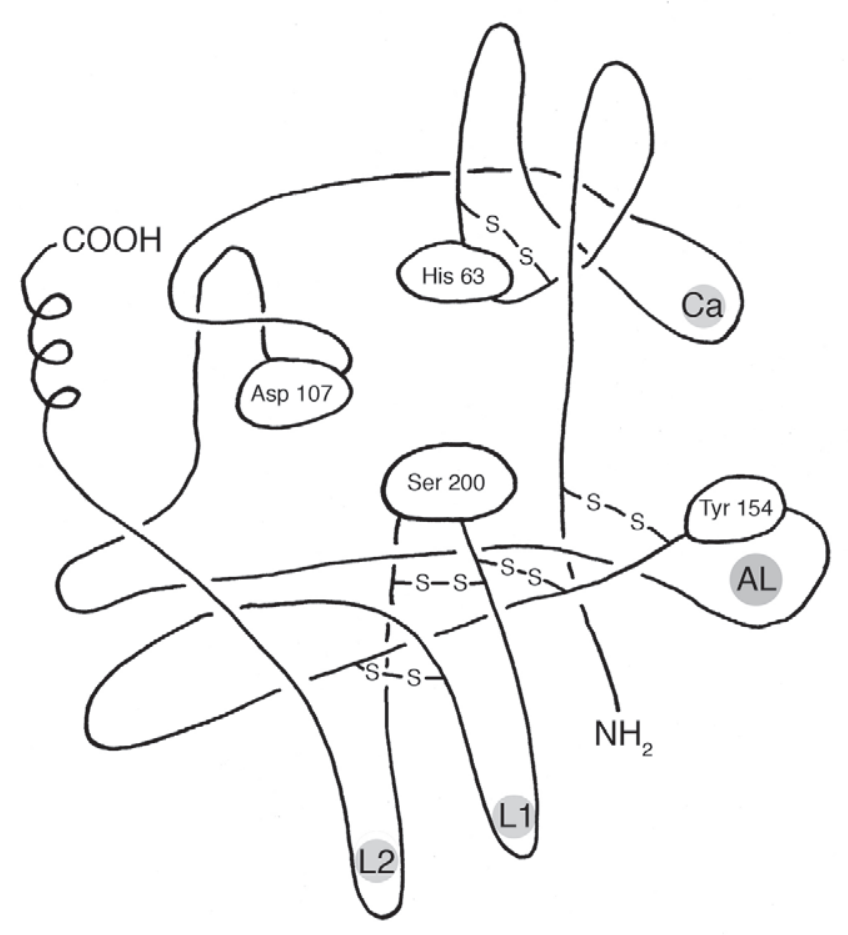

Figure 1. A shematic diagram of human trypsinogen-1. His63, Asp107 and Ser200 form the catalytic triad. L1 and L2, concerved loops which control the specificity of trypsin; AL, autolysis loop; $\mathrm{Ca}$, calcium binding loop; -S-S-, disulfide bond.

trafficing and sorting to a lysosome or a zymogen granule.

Premature and intracellular activation of trypsinogen in experimental pancreatitis has been shown to depend on the presence of CTSB in CTSB deficient mice (Halangk et al., 2000), isolated rat pancreatic acini (Halangk et al., 2002, Saluja et al., 1997) and pancreatic homogenates from mice and rats (Van Acker et al., 2002). Asn29Ile mutation in trypsinogen-1 is associated with HP (Gorry et al., 1997). As compared to native human trypsinogen-1, the activation rate of recombinant Asn29Ile trypsinogen-1 by CTSB is increased threefold even in the presence of PSTI. This suggests that activation of trypsinogen by CTSB may play a role in the development of human pancreatitis (Szilagyi et al., 2001). However, there are several findings incompatible with the so called cathepsin B hypothesis
(Klonowski-Stumpe et al., 1998, Lerch et al., 1993, Teich et al., 2002, Tooze et al., 1991), so the physiological and the pathophysiological role of CTBS as trypsinogen activator remains speculative.

\section{Factors affecting trypsinogen activation}

pH and calcium concentration. Autoactivation of recombinant human trypsinogens has been shown to be $\mathrm{pH}$ - and calcium-dependent in vitro (Kukor et al., 2003). Acidic $\mathrm{pH}$ stimulates autoactivation of recombinant human trypsinogen-1, but inhibits that of trypsinogen-2. At $\mathrm{pH} 8$ in the presence of calcium at low concentration $(<1 \mathrm{mmol} / \mathrm{L})$ trypsinogen-2 exhibits minimal autoactivation due to rapid zymogen degradation, whereas trypsinogen-1 autoactivation is stimulated in a calcium concentration-dependent manner. Increasing 
the calcium concentration progressively inhibits trypsinogen-1 autoactivation. A similar effect on trypsinogen-1 can be seen at high ionic strength $(100 \mathrm{mmol} / \mathrm{L} \mathrm{NaCl})$. In contrast, calcium at $5 \mathrm{mmol} / \mathrm{L}$ stimulates autoactivation and inhibits autodegradation of trypsinogen-2. The effect of high $\mathrm{NaCl}$ concentrations on trypsinogen-2 was less significant.

During CCK hyperstimulation, the apical pole of isolated mouse acinar cells undergo a $\mathrm{Ca}^{2+}$-dependent change characterized by local trypsin activation and replacement of the normal zymogen granules by vacuoles (Raraty et al., 2000). Sustained increase in cytosolic $\mathrm{Ca}^{2+}$ concentration has been demonstrated by an ion exchange mechanism to increase the free $\mathrm{Ca}^{2+}$ concentration and decrease the $\mathrm{pH}$ in the zymogen granules of mouse acinar cells resulting in premature activation and stabilization of trypsin (Yang et al., 2007).

The N-terminal sequence of four Asp residues has a negative effect on the hydrolysis of the Lys-Ile bond by trypsin as studied by using bovine and porcine trypsin (Abita et al., 1969). However, the slow hydrolysis of the Lys-Ile bond by trypsin is accelerated by calcium binding to the Asp residues of the activation peptide by decreasing the $\mathrm{K}_{\mathrm{m}}$ of the reaction, i.e. improving the binding of trypsinogen to trypsin. This effect is mediated by neutralizing the high concentration of negative charges of the activation peptide (Abita et al., 1969, Nemoda and Sahin-Tóth, 2005).

Chymotrypsin $C$. The trypsinogens liberate the eight AA activation peptide Ala-Pro-PheAsp-Asp-Asp-Asp-Lys upon activation. In addition, a pentapeptide Asp-Asp-Asp-AspLys is also formed from trypsinogen-1 (Guy et al., 1978, Nemoda and Sahin-Tóth, 2006). Chymotrypsin C (or caldecrin) specifically cleaves the Phe18-Asp19 peptide bond in the trypsinogen activation peptide removing the N-terminal tripeptide(NemodaandSahin-Tóth, 2006). Autoactivation of this N-terminally truncated trypsinogen-1 is stimulated 3 -fold. This effect is dependent on the presence of Asp218, which forms part of the S3 subsite on trypsin. The N-terminal truncation of trypsinogen- 1 is presumed to result in a conformational change within the remainder of the activation peptide, which repositions Asp21 and thereby mitigates the Asp21Asp218 electrostatic repulsion (Nemoda and Sahin-Tóth, 2006). As chymotrypsinogen C is activated by trypsin, this reaction establishes a novel positive feedback mechanism in the digestive enzyme cascade of humans. The hereditary pancreatitis (HP) -associated mutation Ala16Val in trypsinogen-1 increases the rate of chymotrypsin $\mathrm{C}$ processing of the activation peptide four-fold and causes accelerated trypsinogen-1 activation in vitro. Chymotrypsin Calso cleaves off the N-terminal tripeptide from human trypsinogen-2, but it has no significant effect on the autoactivation trypsinogen-2, which contains Tyr in place of Asp218 (Nemoda and Sahin-Tóth, 2006). The corresponding residue in trypsinogen-3 is His 218 .

Sulfation. As shown by us (IV) and others (Sahin-Tóth et al., 2006, Scheele et al., 1981), pancreatic trypsinogens are sulfated at Tyr154, which together with His46 lines the S'2 binding site (Gaboriaud et al., 1996, Schellenberger et al., 1994). This negatively charged modification has been proposed to modify interactions between trypsin and various substrates and inhibitors (Gaboriaud et al., 1996, Szilagyi et al., 2001). Even though the catalytic activity of sulfated pancreatic and non-sulfated recombinant trypsin-1 are essentially identical, the autoactivation of sulfated pancreatic trypsinogen-1 is 1.4 -fold faster in the presence of $1 \mathrm{mmol} / \mathrm{L} \mathrm{Ca}^{2+}$ and 2.4-fold faster in the presence of in 10 $\mathrm{mmol} / \mathrm{L} \mathrm{Ca}^{2+}$ than that of the non-sulfated recombinant form. In contrast, autoactivation of trypsinogen-2 is unaffected by Tyr 154 sulfation (Sahin-Tóth et al., 2006).

Structural features. There are several structural features in the trypsinogen molecule that regulate its activation. The stability of the zymogen and the slow hydrolysis of the Lys23-Ile24 bond seem to be important mechanisms of protection against accidental 
activation (Abita et al., 1969). Biochemical characterization of pancreatitis-associated activation peptide mutations in human trypsinogen-1 confirmed the importance of Asp residues in the activation peptide for control of autoactivation (Chen et al., 2003a, Teich et al., 2000). Suppression of autoactivation by electrostatic repulsion between the Asp residues in the activation peptide and the surface of trypsinogen-1 (Asp218 in the S3-S4 subsite) is further supported by sitedirected mutagenesis in recombinant human trypsinogens (Chen et al., 2003a, Nemoda and Sahin-Tóth, 2005). Moreover, trypsin has up to eleven-fold preference for Arg over Lys at the P1 position of peptide substrates (Craik et al., 1985, Hedstrom et al., 1996), so use of Lys instead of Arg at the scissile bond also protects against autoactivation. The neutralizing effect of the high concentration of negative charge at the activation peptide by calcium binding provides yet another regulatory element (Nemoda and Sahin-Tóth, 2005).

\section{Trypsinogen and trypsin structure and mechanism of catalysis}

\section{Structure}

The crystal structures of human pancreatic trypsin-1 (Gaboriaud et al., 1996), brain trypsin-4 (Katona et al., 2002), trypsin and trypsinogen from bovine (Bode et al., 1976, Fehlhammer et al., 1977, Finer-Moore et al., 1992, Huber et al., 1974, Kossiakoff et al., 1977, Stroud et al., 1974) and from other species (Huang et al., 1994, Perona et al., 1993) have revealed highly conserved structural motifs within the trypsin family, the structural basis of substrate specificity as well as the mechanism of catalysis. Structurally, the trypsinogen molecule consist of two sixstranded beta barrels and the active site cleft is located between the two barrels (Figure 1).

The trypsinogen molecule consists of different functional domains; catalytic, substrate recognition and zymogen activation domains. However, the functional processes are not separate. More detailed domains that can be characterized within the mentioned ones are the activation peptide (Ala16 to Lys23), the calcium binding loop (Glu75 to Glu85), autolysis loop (Gly145 to Asp156) and oxyanion hole (backbone NHs of Gly198 to Ser200). In addition, up to 200 water molecules both inside the trypsin(ogen) molecule and on its surface are important in serving hydrogen bonds to stabilize the three-dimensional structure of trypsinogen and trypsin and to participate in the catalytic reaction. Internal water clusters are well conserved in various trypsin(ogen)s, and they are frequently shaped as water channels forming extensive hydrogen-bonding networks linked to the protein backbone (Bartunik et al., 1989, FinerMoore et al., 1992, Krem and Di Cera, 1998, McDowell and Kossiakoff, 1995).

The eight AA activation peptide of trypsinogens and Asp199 stabilize the inactive trypsinogen conformation (Pasternak et al., 1998). Approximately $85 \%$ of the structures of trypsinogen and trypsin are identical (Fehlhammer et al., 1977, Kossiakoff et al., 1977) and trypsinogen (and chymotrypsinogen) has weak intrinsic activity towards small active site titrants (Kerr et al., 1975). The oxyanion hole, which is important for stabilization of the tetrahedral intermediate in the catalysis, and the primary binding site of trypsinogen are deformed, which renders the zymogen inactive. Upon cleavage of the activation peptide, the $\alpha$-amino group of the new N-terminal Ile24 folds into a pocket and forms a buried salt bridge between the carboxylate group of Asp199 (Robinson et al., 1973), a mechanism coined "molecular sexuality" (Bode and Huber, 1976). However, hydrophobic interactions of the Ile24 sidechain provide the more stabilization energy for the trypsinogen to trypsin conversion that the salt bridge (Hedstrom et al., 1996). The resulting $170^{\circ}$ rotation of the Asp199 side chain triggers a conformational change in the S1 binding site and oxyanion hole, which produces active enzyme (Fehlhammer et al., 1977). The Ile-Val dipeptide, analogous to the N-terminus of active trypsin, can also cause activation of trypsinogen without 
cleavage of the activation peptide. This data indicates that zymogen activation is based on a conformational change (Bode and Huber, 1976).

\section{Substrate binding}

The substrate recognition sites include the polypeptide binding site, the binding pockets for the side-chains of the peptide substrate and surface loops outside the substrate binding pocket (Hedstrom et al., 1992). The nomenclature of the binding sites is based to that proposed by Schechter and Berger, where P1-P1' denotes peptide residues on the acyl and leaving group side of the scissile bond, respectively (Schechter and Berger, 1967). The adjacent peptide residues are numbered outward, and the S1, S1' etc. denote the corresponding enzyme binding sites.

The primary substrate-binding pocket. The disulfide bond Cys196 - Cys220, and the segments between Asp194 - Asp199, Ser215 - Cys220 and Pro226 - Tyr229 form the primary substrate-binding pocket called S1 binding site in active trypsin (Fehlhammer et al., 1977, Varallyay et al., 1997). The substrate specificity towards peptide bonds following Arg or Lys is mainly defined by three conserved residues; Asp194 at the bottom of the substrate binding pocket and Gly217 and Gly227 residues, which together create the negatively charged S1 site (Huber et al., 1974, Perona et al., 1995). The S1-P1 interaction dominates over substrate binding in the S2 to S4 sites (Sichler et al., 2002). Trypsin prefers Arg substrates over Lys substrates because Arg and Lys interact with the substrate binding pocket in different modes. The cyclic network of hydrogen bonds between the guanidinium group of P1 Arg and S1 Asp194 is the dominant feature of Arg substrate specificity. The chemical characteristics of the side-chain of Ser195 affects the specificity of trypsin towards P1 Lys through a critical hydrogen bond triad involving a water molecule, Ser195 $\mathrm{O} \delta$ and the substrate P1 Lys N $\zeta$ (Evnin et al., 1990). Substrate Lys is indirectly hydrogen bonded to the S1 site Asp194 via a water molecule.

The oxyanion hole. Gly198 in the oxyanion hole also plays a basic role in substrate binding by stabilizing the ground state and the transition state (Bobofchak et al., 2005). Gly198 is highly conserved in serine proteases, but in human trypsin-3 and brain trypsin- 4 the residue at position 198 is Arg (Nyaruhucha et al., 1997). The conformation of the Arg 198 side-chain prevents correct positioning of the amido hydrogen to form a hydrogen bond with the substrate. This feature together with His instead of Asp in position 218 is believed to provide the structural basis for the enhanced inhibitor resistance and binding affinity of substrates for human trypsin-3 and -4 (Katona et al., 2002).

The polypeptide binding site. The polypeptide binding site refers to the main chain of residues Ser215 -Asp218 which form an antiparallel beta sheet with the backbone of the P1 - P3 residues of peptide substrates. The beta sheet structure causes the side chains of the peptide substrate to point in opposite directions (Hedstrom, 2002, Sweet et al., 1974).

Loop structures. Outside the substrate binding pocket near the S1 binding site are two conserved loops called L1 and L2, respectively, which control the specificity of trypsin. These surface loops connect the walls of the S1 binding pocket and stabilize the transition state for hydrolysis by improving the orientation of bound substrates relative to the catalytic site (Hedstrom et al., 1994a). In addition, Tyr175 in a third surface loop has been identified as an additional specificity determinant (Hedstrom et al., 1994b, Perona et al., 1995). This kind of extended substrate binding accelerates catalysis.

It is thought that substrate discrimination occurs during the acylation step rather than during substrate binding. The structural basis for substrate discrimination in the acylation step is the ability of 1) P1-Arg or Lys to make favourable electrostatic interactions with Asp194 to enhance the accurate positioning of 
Gly217 and of 2) loop L2 to uniquely specify the conformation of the conserved Gly217, which forms two main-chain hydrogen bonds with the P3 residue of the substrate promoting accurate scissile bond positioning in a discriminatory way (Hedstrom et al., 1992, Hedstrom et al., 1994a, Ma et al., 2005, Perona et al., 1995). In addition, there are five loops designated A to E. Loop C contacts the extended substrate on the $\mathrm{N}$-terminal side of the scissile bond, whereas loops A, B, D and E interact on the leaving group side (Perona and Craik, 1997). Thus, S1 binding site contributes to substrate specificity for ester hydrolysis, whereas specific amide hydrolysis requires both the proper S1 binding site and more distal interactions such as with the loops next to the substrate binding pocket (Hedstrom et al., 1992).

The leaving group side interactions. The leaving group side interactions $\mathrm{S}^{\prime}-\mathrm{P}$ ' are determined by surface loops (see above) (Bode and Huber, 1992, Perona and Craik, 1997). The role of S'1 to S'3 in substrate binding and catalysis in rat trypsin has been studied by active site mapping using nucleophile mixtures (Schellenberger et al., 1994). The most important contact in the S' subsites is a hydrogen bond between main-chain carbonyl oxygen S'2 - main-chain $\mathrm{NH}$ of the P'2 residue, where trypsin prefers positively charged residues. The P'1 and P'3 side-chains point in one direction and the P'2 side-chain in the opposite direction. Large amino acids residues in P'1 and P'3 can probably form contacts with the same region on the enzyme surface and most likely compete for contacts on the enzyme surface. In contrast, positive cooperativity is observed for specific P'2 and P'3 residues, as the P'2 and P'3 side-chains point tin opposite directions.

The P'2 side-chains bind to a region on the trypsin surface that is lined by His46 and Tyr154. Interestingly, in human pancreatic trypsin-1 and trypsin-2 Tyr154 is sulfated (Gaboriaud et al., 1996, Sahin-Tóth et al., 2006, Szilagyi et al., 2001) (IV). This feature, together with Asp at residue 218 (S4 site) is suggested to influence the selective binding of Kazal-type inhibitors to human trypsin-1 (Gaboriaud et al., 1996).

\section{Catalysis}

The physiological reaction catalyzed by trypsin is hydrolysis of peptide bonds on the carboxyl-terminal side of either arginine or lysine. Chemically the reaction is acyl transfer, in which trypsin stabilizes the tetraedral transition state typical to this reaction (Kraut, 1977). The mechanism is a base-catalyzed nucleophilic attack of the hydroxyl-O of Ser200 to the carbonyl-C of the substrate (Craik et al., 1987, Weiner et al., 1986). The so called catalytic triad or charge relay system - His63, Ser200 and Asp107 - is essential for the catalysis. It is part of an extensive hydrogen bonding network within the enzyme itself and with the substrate during catalysis. The strength of the hydrogen bonds changes during catalysis (Fodor et al., 2006).

Formation of a Michaelis complex. AMichaelis complex is formed upon substrate side-chain binding to the binding pocket: a hydrogen bond between the $\mathrm{O} \gamma$ of Ser200 and N 22 of His63 in the active site becomes sterically optimal for hydrogen transfer as the result of reorganization in the side-chain of Ser200 and movement of the imidazole ring of His63 (Ruhlmann et al., 1973). Then the Ser200 O $\gamma$ can form a covalent bond with substrate and donate a proton to His63. In addition, the sidechain of the substrate Lys residue becomes hydrogen-bonded to Asp194 via a water molecule. The longer side-chain of Arg in the substrate replaces the water molecule in the binding pocket and forms a direct hydrogen bond to Asp194 of trypsin (Bode et al., 1984, Craik et al., 1985, Weber et al., 1995).

The acylation step. After formation of the non-covalent Michaelis complex catalysis is then thought to proceed in two steps, which are simplified as follows. First, acylation of trypsin occurs by the nucleophilic attack of hydroxyl-O of Ser200 to the substrate P1 carbonyl-C resulting in a covalent bond. At 
the same time, the hydroxyl-proton of Ser200 is transferred to the His63 N\&2 base. Hydrogen bonds are formed between the oxyanion hole and $\mathrm{P} 1$ carbonyl-C resulting in polarization of the carbonyl bond. All these bonds force the substrate to a tetrahedral oxyanion intermediate. Then, the imidazole ring of His63 can donate a proton to the leaving group amine on the substrate and the scissile bond is lengthened and broken. Trypsin acylation is most likely the rate-limiting step of the catalysis (Kossiakoff and Spencer, 1980, Kossiakoff and Spencer, 1981, Weiner et al., 1986). However, the deacylation step is only $>3$-fold faster than acylation (Bobofchak et al., 2005).

The deacylation step. After acylation a water molecule located in the catalytic site forms a hydrogen bond with His63. The water molecule acts as nucleophile by attacking the acyl-trypsin P1 carbonyl-C and donates a proton to His63. The formed new tetrahedral transition state breaks down as a proton is transferred from His63 to Ser200 and native trypsin is released (Weiner et al., 1986). The function of negatively charged Asp107, which is located on the opposite side of His63 in the active site, is believed by hydrogen bonding to force His63 in the proper tautomeric state, so that its proton is at $\mathrm{N} \delta 1$ and not at $\mathrm{N} \varepsilon 2$ (Ash et al., 1997, Frey et al., 1994, Kossiakoff and Spencer, 1981, Sprang et al., 1987). Amide bonds are very stable due to electron donation from the amide nitrogen to the carbonyl. It is estimated, that the rate of peptide bond hydrolysis is increased about $10^{10}$-fold by serine protease catalysis as compared to the corresponding uncatalyzed reactions (Hedstrom, 2002).

\section{Functions of trypsins}

\section{Digestion of food}

Digestion of food is the main physiological function of pancreatic trypsin. Indeed, trypsin and chymotrypsin are considered the major workhorses of digestion. Trypsin-1 and -2 isoenzymes degrade dietary proteins in the duodenum either directly or indirectly by activation of other digestive enzymes such as chymotrypsinogen, procarboxypeptidase, phospholipase and proelastase (Neurath and Walsh, 1976, Travis and Roberts, 1969). Trypsin-3 does not activate zymogens, and thus degradation of dietary trypsin inhibitors appears to be the only role of pancreatic trypsin-3 (Sahin-Tóth, 2005, Szilagyi et al., 2001, Szmola et al., 2003) .

Intestinal alkaline sphingomyelinase digests dietary sphingomyelin generating multiple lipid messengers such as ceramide and sphingosine. Pancreatic trypsin has been shown to release intestinal alkaline sphingomyelinase from rat intestinal mucosa in vivo, thereby increasing the enzyme activity about 50 to $70 \%$ (Wu et al., 2004). The trypsininduced dissociation was rapid and specific. By this means pancreatic trypsin would not only digest dietary proteins but indirectly also sphingomyelin.

\section{Activation of protease-activated receptors}

Protease-activated receptors (PAR-1, PAR-2, PAR-3 and PAR-4) are G protein-coupled receptors with seven transmembrane-spanning domains (Dery et al., 1998). PAR-2 is activated by trypsin-like enzymes like trypsin itself, acrosin and mast cell tryptase, whereas PAR-1, PAR-3 and PAR-4 are activated mainly by thrombin (Cottrell et al., 2003, Coughlin, 2005, Fox et al., 1997, Molino et al., 1997, Nystedt et al., 1994). To a lesser extent, human tissue kallikreins, cathepsin $\mathrm{G}$, plasmin, granzyme $\mathrm{A}$, and coagulation factors VIIa and Xa are able to activate PARs (Oikonomopoulou et al., 2006, Vergnolle et al., 2003). The PARs are irreversibly activated by proteolytic cleavage at the amino-terminal exodomain of the receptor. The new, unmasked amino terminus functions as a tethered ligand, docking intramolecularly with the body of the receptor to effect transmembrane signalling. Once ligated, PAR can activate intracellular $G$ proteins and thus mediate extracellular signals to intracellular signalling pathways. Like other G protein-coupled receptors, PAR 
signalling is rapidly attenuated by receptor desensitization, endocytosis, and/or receptor down-regulation (Grady et al., 1997, Ludeman et al., 2004). Trypsin has also been shown to affect PAR-2 ubiquitination, which is required for lysosomal trafficking of PAR-2 (Cottrell et al., 2003).

Protease-activated receptor-2. The gene encoding human PAR-2 has been cloned (Bohm et al., 1996) and PAR-2 has been found to be highly expressed in the pancreatic duct cells, kidney, intestine, liver, prostate, ovary, testes, heart, lung, skin, bladder, brain, and trachea, where it is found in epithelial and endothelial cells, and myocytes, fibroblasts, immune cells, neurons and glial cells (Bohm et al., 1996, D'Andrea et al., 1998, D'Andrea et al., 2000, D'Andrea et al., 2001, Macfarlane et al., 2001, Nguyen et al., 1999, Nystedt et al., 1994, Nystedt et al., 1995). Several functions of the PARs are involved in regulation of hemostasis, inflammation, pain, and tissue repair (Macfarlane et al., 2001). Functional PARs including PAR-2 have also been described in the central and peripheral nervous system suggesting regulative role for PARs and their activating proteases in various processes of the nervous system such as motor, secretory, vascular, nociceptive, inflammatory or regenerative processes (Vergnolle et al., 2003). PAR-2-mediated effects include increase in intracellular $\mathrm{Ca}^{2+}$, effects of ion transport, cell proliferation, growth and adhesion, apoptosis, secretion, immunomodulation and mitogensis. PARsignaling involves molecules like $\mathrm{G} \alpha_{\mathrm{i}}, \mathrm{G} \alpha_{\mathrm{q}}$, phospholipase $\mathrm{C} \beta$ (PLC $\beta$ ), diacylglycerol (DAG), inositoltriphosphate $\left(\mathrm{IP}_{3}\right), \mathrm{NF} \kappa \mathrm{B}$, c-Fos, c-Jun, p38, and extracellular-signal regulated kinases (ERK1/2) (Steinhoff et al., 2005).

Pancreatic trypsins and PAR-2. Pancreatic trypsin-1 and -2 are potent activators of PAR-2, which is present at high densities on the luminal surfaces of pancreatic acinar cells, duct epithelial cells, and the intestine (Kong et al., 1997, Nguyen et al., 1999). PAR-2 activation stimulates cytokine production and regulates pancreatic exocrine function via a negative feedback loop (Hirota et al., 2006a, Maeda et al., 2005). Physiological concentrations of trypsin in the intestinal lumen $(100 \mathrm{nmol} / \mathrm{L})$ activates PAR-2 at the apical membrane of enterocytes and stimulates the generation of $\mathrm{IP}_{3}$, arachidonic acid release and prostaglandin secretion (Kong et al., 1997).

In cultured dog pancreatic duct epithelial cells, trypsin can activate ion channels by cleaving and triggering PAR-2, which results in increased intracellular calcium concentration and subsequent stimulation of $\mathrm{Ca}^{2+}$-activated $\mathrm{Cl}^{-}$and $\mathrm{K}^{+}$channels (Nguyen et al., 1999). In the gastrointestinal track, PAR-2 mediated contractile responses have been reported, most likely via a mechanism involving $\mathrm{Ca}^{2+}$ dependent $\mathrm{K}^{+}$channels (Cocks et al., 1999b). Furthermore, PAR-2 has been linked to the release of amylase from the acinar cells of the pancreas, and exocrine secretion from salivary, parotid and sublingual glands (Bohm et al., 1996, Kawabata et al., 2000, Kawabata et al., 2002, Nguyen et al., 1999). Thus, besides acting as digestive proteinase and activator of other digestive enzymes, pancreatic trypsin is also a signalling molecule regulating cells of the gastrointestinal track by activation of PAR-2.

Extra-pancreatic trypsins and PARs. Trypsin-4 has been suggested to activate PAR-2 and -4 and the inhibitor resistance of trypsin-4 has been postulated to promote prolonged PAR-mediated signaling in extra-pancreatic cells (Cottrell et al., 2004). Tumor-derived human epithelial cell lines from prostate (PC-3), colon (SW480 and Caco2), and airway (A549) have been found to express PAR-2, trypsinogen-4 and enteropeptidase. Expression of trypsinogen- 4 and its activation by enteropeptidase induces a prompt increase in intracellular calcium in KNRK cells (a normal rat kidney [NRK] cell line transformed by Kirsten murine sarcoma virus) expressing human PAR-2, but not in nontransfected cells, suggesting that trypsin-4 is an activator of PAR-2 (Cottrell et al., 2003, Cottrell et al., 2004). However, studies with recombinant 
trypsin isoforms revealed that the activity of trypsin-4 was completely unable to activate epithelial PAR-1 and-2. Instead, it weakly activated brain PAR-1 in human astrocytoma 1321N1 cells (Grishina et al., 2005).

Results from another group (Wang et al., 2006) revealed that trypsin-4 selectively induces transient $\mathrm{Ca}^{2+}$ mobilization in both rat astrocytes and retinal ganglion RGC-5 cells via activation of PAR-1. The activating cleavage site is Arg-Ser in PAR-1 and PAR-2, Lys-Thr in PAR-3, and Arg-Gly in PAR-4. Arg-Ser and Lys-Thr peptide bonds are readily cleaved by trypsin-3 and -4, so PAR-1, PAR-2, and PAR-3 are potential trypsin-4 substrates (Szepessy and Sahin-Tóth, 2006).

\section{Trypsinogens in cancer}

Proteolytic processing of ECM. As discussed above, trypsin degrades many ECM components (Koivunen et al., 1991a, Koshikawa et al., 1992, Moilanen et al., 2003, Stenman et al., 2005) but it is also a potent activator of several MMPs (Imai et al., 1995, Koivunen et al., 1989, Moilanen et al., 2003, Nyberg et al., 2002, Paju et al., 2001b, Sorsa et al., 1997, Umenishi et al., 1990). In addition to breakdown of ECM components and activation of proteinase cascades tumor-associated trypsins can modulate cancer cells by other mechanisms, too. Proteolytic processing of ECM exposes cryptic binding sites within ECM molecules, generates biologically active ECM fragments and affects the bioavailability and activity of sequestered growth factors and receptors (Liotta and Kohn, 2001).

Tumor-associated trypsin and PAR-2. Recent studies suggest a signalling function for tumorassociated trypsin as well as other proteinases. The binding of integrins to ECM proteins activates focal adhesion kinases (FAKs). These in turn interact with several intracellular signalling molecules. Stimulation of cellular growth, adhesion to fibronectin and vitronectin, and, when transplanted to nude mice, tumor production of human gastric carcinoma cells overexpressing trypsinogen-1 suggests that trypsin- 1 contributes to disseminated growth of some cancer cells (Miyata et al., 1998). Integrin $\alpha_{5} \beta_{1}$-dependent cellular adhesion to fibronectin and proliferation of MKN-1 human gastric carcinoma cells was shown to be regulated by PAR-2 and $G$ protein signalling induced by tumor-associated trypsin (Miyata et al., 2000).

Trypsin has been shown to be a potent growth factor for human colon cancer cells in vitro and the action is mediated by activation of PAR-2 and subsequent increase in intracellular $\mathrm{Ca}^{2+}$ concentration (Darmoul et al., 2001, Ducroc et al., 2002). The mechanism is dependent on MMP-mediated release of transforming growth factor- $\alpha$ (TGF- $\alpha)$, transactivation and phosphorylation of epidermal growth factor receptor (EGF-R) and subsequent activation of extracellular signal-regulated protein kinase $1 / 2$ (ERK1/2) and cell proliferation (Darmoul et al., 2004). On the other hand, trypsinogen-4 has been hypothesized to possess a tumorsuppressive role in cancer progression as it has been shown to be silenced at the mRNA level by promoter methylation in several gastric adenocarcinomas and esophageal squamous cell carcinomas (Yamashita et al., 2003).

Trypsin-2 isolated from a colon carcinoma cell line has been shown to be more potent activator of PAR-2 than two different mast cell tryptases and almost equally effective as bovine pancreatic trypsin in an in vitro study (Alm et al., 2000). Interestingly, the PARs are up-regulated in cancer and inflammation (Borgono and Diamandis, 2004). Taken together, tumor-associated trypsins are potential in vivo activators of PAR-2.

Effects on the surrounding cells. Cells in the tumor microenvironment, i.e. mast cells, macrophages, endothelial cells, and vascular smooth muscle cells have been shown to express PAR-1 and PAR-2. These cells may act as proteolytic sensors to extracellular thrombin and trypsin, respectively, and thus enable a permissive environment for tumor growth and metastasis via an autocrine and/or paracrine cascade. PAR-1 and PAR-2 are also 
detected on stromal fibroblasts surrounding metastatic tumor cells but not on fibroblasts surrounding benign, non-metastatic or normal epithelial cells (D’Andrea et al., 2001).

Angiogenesis. Tumor cell-activated endothelial cells produce trypsin, which has been suggested to contribute to tumor angiogenesis and tumor metastasis by activation of matrix metalloproteinases (MMPs) or direct matrix degradation (Koshikawa et al., 1997). On the other hand, many angiogenesis inhibitors are stored as cryptic fragments within larger precursor matrix molecules, and the regulation of proteolytic processing of extracellular matrix plays an important role in vascularization of tumors (Nyberg et al., 2005).

\section{Other functions of trypsins}

Paneth cells. The selective antibiotic activity of Paneth cell human $\alpha$-defensin 5 and 6 (HD5 and HD6) is enhanced by tryptic processing. Unlike in the pancreas, only trypsin-2 and -3 are expressed at a 6:1 ratio in the Paneth cells of the small intestine, suggesting that Paneth cells express a distinct trypsin isoform pattern. Paneth cell-derived trypsin is suggested to be the processing proteinase of HD5 in vivo and trypsin activity seems to be carefully regulated by API and PSTI that also are present in the Paneth cells (Ghosh et al., 2002). Paneth cell trypsin could also be the activator of PAR-2 expressed on luminal surfaces of enterocytes of the human intestinal crypts.

Genital tract. Trypsin is widely distributed in the male genital tract and may play a physiological role in semen. Trypsin purified from human seminal fluid activates the proform of prostate specific antigen (PSA) (Paju et al., 2000), which cleaves semenogelins I and II in the sperm-entrapping gel forming after ejaculation (Lilja, 1985).

Central nervous system. Human trypsin-4 has recently been shown to selectively process two Arg-Thr peptide bonds in human myelin basic protein, which is the most abundant membrane protein in the central nervous system and an autoantigen in multiple sclerosis (Medveczky et al., 2006). Trypsin-4 has also been implicated in the increased production of glial fibrillary acidic protein (GFAP) and accumulation of $\beta$-amyloid in the brain of transgenic mice expressing trypsinogen- 4 in neurons (Minn et al., 1998). However, the possible role of trypsin- 4 in neurodegenerative diseases remains to be elucidated.

Ion channels. Acid-sensing ion channels (ASICs) are non-voltage-gated $\mathrm{Na}^{+}$channels of the epithelial $\mathrm{Na}^{+}$channel/degenerin family. They are almost ubiquitous in the mammalian nervous system and they are transiently activated by a rapid drop in extracellular $\mathrm{pH}$ (Krishtal,2003). Several putative physiological roles of ASICs have been proposed, like pain receptor, modulation of synaptic transmission, memory and fear conditioning and mediation of cell injury in acidosis. Trypsin has been shown to cleave ASIC 1a in the N-terminal part of an extracellular loop in vitro, thereby shifting the $\mathrm{pH}$-dependence of channel activation and inactivation to more acidic $\mathrm{pH}$ (Vukicevic et al., 2006). Trypsin has been demonstrated in vitro to cleave $C$ termini of $\beta$ - and $\gamma$-subunits of epithelial $\mathrm{Na}^{+}$channels $(\mathrm{ENaC})$. This is believed to increase $\mathrm{ENaC}$ activity and be one of the physiological mechanisms of sodium channel regulation (Jovov et al., 2002).

Leucocyte adhesion. Trypsin has been reported to up-regulate the intercellular adhesion molecule-1 (ICAM-1), a key vascular endothelial adhesion molecule necessary for transport of leukocytes from the intravascular space into inflamed tissues (Hartwig et al., 2004). Up-regulation by trypsin occurs both in the rat pancreas and lung and it is associated with increases in leukocyte infiltration into the tissues and decreased perfusion of pancreatic microvasculature.

Airways. In preterm infants, the development of bronchopulmonary dysplasia (BPD) is associated with high pulmonary concentrations of trypsinogen-2 during the first two postnatal weeks (Cederqvist et al., 2003). In addition, infants with higher trypsinogen-2 to TATI 
ratio subsequently developed BPD. The underlying mechanism remains unclear, but it has been suggested that trypsin degrades ECM directly, activates latent MMPs, or mediates inflammatory reactions via activation of PAR-2. Bronchial epithelial cells express both trypsin(ogen) and PAR-2 (Cocks et al., 1999a). Indeed, trypsin released from the epithelium can initiate brochorelaxation in the airways by activation of epithelial PAR-2 and is thus hypothesized to participate in prostanoid-dependent cytoprotection in the airways (Cocks et al., 1999a).

Other functions. Trypsin has been shown to participate in cardiovascular events via PAR-2 in animal models, but the physiological and pathophysiological role remains unclear (Macfarlane et al., 2001). In the skin, activation of PAR-2 by trypsin has been linked to pigmentation via action of prostaglandins and their receptors (Scott et al., 2004). In rat brain, trypsin has been shown to cleave the virus envelope fusion glycoprotein precursor hemagglutinin $\left(\mathrm{HA}_{0}\right)$ of human influenza $\mathrm{A}$ virus and the fusion glycoprotein precursor $\left(\mathrm{F}_{0}\right)$ of Sendai virus (Le et al., 2006). After virus infection in rat lungs the levels of TNF- $\alpha$, trypsin-1 and MMP-9 mRNA, respectively, were significantly up-regulated (Yamada et al., 2006). These results suggest that trypsin in the brain might potentiate virus multiplication and progression of influenzaassociated encephalopathy or encephalitis. Finally, a function as a "pipe-cleaner" has been proposed for trypsin produced by various types of epithelial cells, like those of the bile duct and the nephron of the kidney (Koshikawa et al., 1998).

\section{Trypsin inhibitors}

Several classes of inhibitors mimic the tetrahedral intermediate of the serine protease reaction and form stable tetrahedral adducts with the protease (Kraut, 1977) in a so called "canonical" substrate-like manner, where numerous polar and hydrophobic interactions between the protease and the inhibitor prevent rapid dissociation of the complex. Prolonged association of the enzyme and the inhibitor leads to an equilibrium between the cleaved and uncleaved forms of the inhibitor (Fodor et al., 2005, Fodor et al., 2006). However, not all inhibitors interact "canonically" (Rydel et al., 1990, Rydel et al., 1991), a covalent bond between the enzyme and the inhibitor is not necessary for inhibition, and the proteaseinhibitor complex is not a fully tetrahedral adduct (Baillargeon et al., 1980, Richarz et al., 1980). The canonical inhibitors, like PSTI and API, are unrelated in structure but have in common a "primary binding segment", a flatshaped loop that fits into the active-site cleft of cognate proteinase. All protein inhibitors of proteinases prevent access of (large) substrates to the catalytic site of the enzyme by steric hindrance. Endogenous protease inhibitors appear to be proteins, small non-protein inhibitors are produced by micro-organisms (Bode and Huber, 1992).

The first natural protease inhibitors were identified by Northrop and Kunitz as part of their protease studies in the 1930s with cattle pancreas (Kunitz and Northrop, 1935). An inhibitor was characterized as a polypeptide with a molecular weight of about $6000 \mathrm{Da}$, and that forms a reversible complex with trypsin in a molar ratio of $1: 1$. Today this inhibitor

Table 3. Trypsin inhibitors in human plasma

\begin{tabular}{lccl} 
Inhibitor & $\begin{array}{c}\text { Concentration } \\
(\mathrm{g} / \mathrm{L})\end{array}$ & $\begin{array}{c}\mathrm{MW} \\
(\mathrm{kDa})\end{array}$ & Reference \\
\hline$\alpha_{2}$-macroglobulin & $2-4$ & 720 & (Sottrup-Jensen, 1989) \\
$\alpha_{1}$-proteinase inhibitor & 1.3 & 51 & (Carrell, 1986) \\
Inter- $\alpha$-inhibitors & $0.6-1.2$ & $30-250$ & (Josic et al., 2006) \\
PSTI/TATI & $5-20 \times 10^{-6}$ & 6.2 & (Stenman et al., 1982)
\end{tabular}


is called basic pancreatic trypsin inhibitor (BPTI) or aprotinin, and it belongs to the Kunitz type inhibitor family. Human pancreas does not contain a Kunitz type inhibitor, but both human trypsin-1 and -2 are inhibited by BPTI in a 1:1 molar ratio (Figarella et al., 1975). Protease inhibitors are after albumin and immunoglobulins the third largest group of functional proteins comprising about $10 \%$ of total plasma proteins in vertebrates (Travis and Salvesen, 1983) (Table 3).

\section{$\alpha_{2}$-macroglobulin}

$\alpha_{2}$-macroglobulin $\left(\alpha_{2} M\right)$ and $\alpha_{1}$-proteinase inhibitor (API, also called $\alpha_{1}$-antitrypsin) are the major protease inhibitors in human plasma (Laskowski and Kato, 1980) (Table 3). Human $\alpha_{2} \mathrm{M}$ is a large (MW $720000 \mathrm{Da}$ ) glycoprotein, composed of four identical subunits (SottrupJensen, 1989). It is a non-specific protease inhibitor, which controls the activity of proteinases not only by active site-directed inhibition but also by steric shielding and rapid clearance. Specific limited proteolysis of $\alpha_{2} \mathrm{M}$ at a site called the bait region results in a conformational change in $\alpha_{2} \mathrm{M}$ leaving the protease irreversibely bound to $\alpha_{2} \mathrm{M}$ (Barrett et al., 1979, Borth, 1992, Bretaudiere et al., 1988). One $\alpha_{2} \mathrm{M}$ molecule can trap one or two proteinase molecules (Sottrup-Jensen, 1989). Protease- $\alpha_{2} \mathrm{M}$ complexes are rapidly eliminated from the circulation by LDL-receptor-related protein mediated endocytosis (Sottrup-Jensen, 1989) primarily by hepatocytes (Feldman et al., 1985).

\section{$\alpha_{1}$-proteinase inhibitor}

Apart from $\alpha_{2} \mathrm{M}$, the most abundant human plasma proteinase inhibitors are serpins (serine proteinase inhibitors). The serpins share a conserved structure and employ a unique irreversible suicide substrate-like inhibitory mechanism. Thirty four human serpins, including $\alpha_{1}$-proteinase inhibitor (API) have already been identified (Gettins, 2002). API is the serpin present at the highest concentration in human plasma and it is mainly produced by the liver (Carrell, 1986). API is able to inhibit several serine proteases, but the regulation of neutrophil elastase is considered to be its main physiological function (Beatty et al., 1980, Travis and Salvesen, 1983).

Human API (MW $51000 \mathrm{Da}$ ) forms complex with trypsin in a 1:1 molar ratio. The proteinase first forms a noncovalent Michaelis complex with API. Subsequent peptide bond hydrolysis of the reactive center loop results in formation of acyl-enzyme intermediate and insertion of the reactive center loop into a $\beta$-sheet. Upon complete loop insertion the proteinase is translocated and compressed against the base of API, its active site is grossly distorted and hence inactivated (Huntington et al., 2000, Silverman et al., 2001).

Proteinases complexed to API can be degraded by other proteinases (Kaslik et al., 1995, Stavridi et al., 1996). This may be a faster way of proteinase elimination from the circulation than the SEC (serpinenzyme complex) receptor-based uptake and intracellular degradation of proteinase-API complexes (Perlmutter et al., 1990, Pizzo, 1989, Pratt et al., 1988). API inhibits trypsin-2 ten times faster than trypsin-1, and it has been suggested to control trypsin-1 activity in vivo when $\alpha_{2} \mathrm{M}$ is already saturated (VercaigneMarko et al., 1989). In this case API would have a significant role in the inhibition of trypsin-2 under physiological conditions and of trypsin-1 under pathological conditions.

\section{Inter- $\alpha$-inhibitors}

Inter- $\alpha$-inhibitor proteins comprise a family of serine proteinase inhibitors found at relatively high concentrations in human plasma, i.e. 0.6 to $1.2 \mathrm{~g} / \mathrm{L}$ (Josic et al., 2006). Inter- $\alpha$-trypsin inhibitor (ITI) was first characterized and isolated from human plasma in the 1960s (Heimburger et al., 1964, Steinbuch and Loeb, 1961). It was initially characterized as a zinc-containing glycoprotein that inhibits trypsin and chymotrypsin by forming 1:1 complexes (Aubry and Bieth, 1976). ITI was shown to be structurally related to the Kunitz family of inhibitors and homologous to bovine 
pancreatic trypsin inhibitor BPTI (Wachter and Hochstrasser, 1981), but its antiproteinase function is relatively weak.

The inter- $\alpha$-inhibitor proteins consist of heavy chains, H1, H2, H3 and/or H4 (65, 70, 90 and $120 \mathrm{kDa}$, respectively) and/or a $30 \mathrm{kDa}$ light chain called bikunin. The genes encoding the subunits have been characterized (Nishimura et al., 1995, Salier, 1990). Uncomplexed bikunin, which has two Kunitz-type inhibitory domains, inhibits several serine proteinases including trypsin, plasmin, elastase and cathepsin B (Josic et al., 2006, Salier et al., 1996). The $250 \mathrm{kDa}$ inter- $\alpha$-inhibitor, previously called ITI, contains three subunits, two heavy chains $\mathrm{H} 1$ and $\mathrm{H} 2$ and bikunin. The $125 \mathrm{kDa}$ pre- $\alpha$-inhibitor $(\mathrm{P} \alpha \mathrm{I})$, previously known as pre- $\alpha$-trypsin inhibitor, contains two subunits, heavy chain $\mathrm{H} 3$ and bikunin. (Josic et al., 2006). The chains are covalently bound via a protein - glycosaminoglycan protein bridge, where chondroitin 4-sulfate is the glycosaminoglycan. Bikunin contains an $\mathrm{N}$-linked oligosaccharide and a chondroitin sulfate chain (Josic et al., 2006, Salier et al., 1996).

Studies in mice suggest that ITI acts as a shuttle by transferring proteinases to other plasma proteinase inhibitors like $\alpha_{2} \mathrm{M}$ and API for clearance, and that ITI modulates the distribution of proteinase among inhibitors (Pratt and Pizzo, 1986, Pratt et al., 1987). On the other hand, a so-called von Willebrand type-A, multicopper oxidase and bradykininlike domains have been identified in the heavy chains, suggesting several other functions, like a role in inflammation and maintenance of extracellular matrix stability and integrity through hyalyronic acid-binding (Bost et al., 1998, Salier et al., 1996).

\section{PSTI or TATI}

SPINK1 gene. PSTI is Kazal-type trypsin inhibitor originally purified from bovine pancreas from a side-fraction in a commercial insulin process (Kazal et al., 1948). The sequence of human PSTI was identified in
1977 (Bartelt et al., 1977) and today the single human PSTI gene (serine protease inhibitor Kazal type 1 or SPINK1 gene) has been characterized. It is $7.5 \mathrm{~kb}$ long, separated into four exons and is located on chromosome 5 (Horii et al., 1987).

The genomic PSTI gene has neither the mammalian pancreas-specific common cisacting regulatory sequence (Walker et al., 1983) nor the typical promoter sequences TATA, CAAT nor GC boxes, but the sequences ATAT and CAATCAAT are positioned in the promoter region of the gene (Horii et al., 1987). It has been suggested that the sequence CAATCAATAAC that is present in two novel 5 ' cis-acting elements in the promoter region of the gene functions as a pancreas-specific element (Yasuda et al., 1998). A 40-bp IL-6responsive element, that is conserved among various acute phase genes, has been identified in the PSTI gene in hepatoma cells (Yasuda et al., 1993).

Biochemical properties of PSTI/TATI. The SPINK1 gene product consists of 79 AAs including a 23 AA signal peptide. Mature PSTI is a 56 AA polypeptide with a molecular weight of 6242 Da containing three intra-chain disulphide bridges. PSTI, or tumor-associated trypsin inhibitor (TATI), isolated from urine of a patient with ovarian cancer (see below) was found to be microheterogenous in charge the pI of the main component being 5.8 (Huhtala et al., 1982). Four forms of PSTI have been purified in human pancreatic juice (Kikuchi et al., 1985). PSTI/TATI is cleared from circulation by excretion into urine with a halflife of six minutes (Marks and Ohlsson, 1983). In fact, serum PSTI/TATI can also be used as a marker for renal function (Tramonti et al., 2003).

PSTI is synthetized and secreted together with trypsinogen by pancreatic acinar cells. The molar ratio of trypsinogen to PSTI in human pancreatic juice is about 5:1 (Hirota et al., 2006a, Rinderknecht, 1986, Rinderknecht, 1993) representing an amount equivalent to 0.1 to $0.8 \%$ of the total protein in pancreatic 
juice (Pubols et al., 1974). The reactive site of human PSTI is residue Lys41, that serves as a specific target substrate for trypsin (Bartelt et al., 1977).

Serum levels of PSTI/TATI. TATI was first isolated from urine of an ovarian cancer patient (Stenman et al., 1982) and was later shown to be identical to PSTI (Huhtala et al., 1982). The concentration of TATI in normal serum is 5 to $20 \mu \mathrm{g} / \mathrm{L}$ and that in urine 5 to $50 \mu \mathrm{g} / \mathrm{L}$ as measured by radioimmunoassay. Elevated levels have been observed in urine from patients with ovarian, cervical and endometrial cancer, as well as in the amniotic fluid from 14 to 16 weeks of pregnancy (Stenman et al., 1982). As measured by another radioimmunoassay, serum PSTI level in healthy individuals ranged from 5.4 to 16.0 $\mu \mathrm{g} / \mathrm{L}$ (Kitahara et al., 1980). Normal serum levels of TATI were found in the serum and urine of pancreatectomized patients (Halila et al., 1985) suggesting that pancreatic acinar cells are not the main source of PSTI/TATI in humans.

Inhibition. PSTI is a strong, reversible trypsin inhibitor, which inhibits both trypsin- 1 and -2 in an equimolar ratio. It is gradually degraded and released from trypsin (Figarella et al., 1975, Laskowski and $\mathrm{Wu}, 1953$ ) by cleavage of the peptide bonds Lys41-Ile42, Arg67Gln68, Arg28-Glu29, Arg65-Lys66 and Lys75-Ser76 (Kikuchi et al., 1989, Schneider and Laskowski, 1974, Schneider et al., 1973). Both human and dog PSTI-trypsin complexes dissociated rapidly when added into serum in vitro (Eddeland and Ohlsson, 1978). The released trypsin was mainly bound by serum $\alpha_{2} \mathrm{M}$ and to a lesser extent to API.

Intravenous injection of PSTI-trypsin complexes into dogs resulted in a similar rapid dissociation of the complexes. The major part of the injected radioactive trypsin was bound by $\alpha_{2} \mathrm{M}$ and API. The released PSTI disappeared rapidly from the circulation into urine and into the whole extracellular fluid volume (Eddeland and Ohlsson, 1978). The peptide bonds Lys41-Ile42, Arg67- Gln68,
Arg28-Glu29, and Lys75-Ser76 have also been shown to be cleaved by trypsin-3 (Szmola et al., 2003).

The function of PSTI in the mucus-producing cells in the gastrointestinal tract is suggested to protect the mucus from digestion by luminal proteinases within the stomach and colon and to stimulate epithelial repair (Freeman et al., 1990, Marchbank et al., 1998). PSTI/TATI is also an efficient inhibitor of acrosin (Huhtala, 1984) suggesting a role in reproduction.

In PSTI deficient (Spink3-/-) mice, autophagic degeneration of acinar cells started from day 16.5 after coitus, resulting in rapid onset of cell death in the pancreas and duodenum, and finally death of the test animals 14.5 days after birth (Ohmuraya et al., 2005). The same researchers reported later (Ohmuraya et al., 2006) that trypsin activity could be detected in pancreatic acinar cells of Spink3-/- mice at 0.5 and 1.5 days after birth. On the contrary, trypsin activity was not detected in pancreatic acinar cells of Spink3+/+ and Spink3+/- mice. Thus, the loss of PSTI resulted in failure to control trypsin activation in acinar cells in mice leading to excessive autophagy in the acinar cells.

Extrapancreatic expression. The physiological role of PSTI was initially thought to solely prevent premature activation of pancreatic proteases, especially trypsinogen (Pubols et al., 1974, Rinderknecht, 1986). However, TATI as well as trypsinogen (see above) are also expressed in several other normal tissues like the gastrointestinal tract (Bohe et al., 1986, Bohe et al., 1988, Bohe et al., 1992, Bohe et al., 1997, Freeman et al., 1990, Shibata et al., 1986), gall bladder and biliary tract, breast, kidney and urinary tract, spleen, epithelial cells of the skin, liver, lung, the brain and vascular endothelial cells (Fukayama et al., 1986, Lukkonen et al., 1999, Marchbank et al., 1996) suggesting an important role for both TATI and trypsinogen in tissues other than the pancreas. 
PSTI/TATI in cancer. The increase of serum PSTI/TATI found in connection with malignant diseases is probably caused by production in the cancer cells, but the acute-phase reaction can also contribute. PSTI/TATI has been shown to be expressed in several cancers, including pancreatic, colorectal, gastric, lung, ovarian, renal cell, and bladder cancers (Diggle et al., 2003, Haglund et al., 1986, Higashiyama et al., 1990a, Higashiyama et al., 1990b, Huhtala et al., 1982, Huhtala et al., 1983, Jarvisalo et al., 1993, Lukkonen et al., 1999, Ohmachi et al., 1993, Paju et al., 2004, Paju et al., 2007, Pasanen et al., 1995, Piantino and Arosaio, 1991, Tomita et al., 1987).

TATI has been shown to be prognostic factor in ovarian cancer (Venesmaa et al., 1994), bladder cancer (Kelloniemi et al., 2003), hepatocellular carcinoma (Lee et al., 2007), and renal cell carcinoma (Paju et al., 2001a). The function of TATI in cancer is thought to be the same as in the pancreas, i.e. the inhibition of trypsin produced by the tumor cells (Stenman et al., 1991). The finding that trypsinogen is expressed in both malignant and benign bladder epithelium, whereas TATI expression decreases with increasing stage and grade, suggests balanced expression of trypsinogen and TATI in normal tissue, but disruption of this balance in tumor progression (Hotakainen et al., 2006). Interestingly, high TATI expression in gastric cancer tissue seems to correlate with a favourable prognosis for the patient (Wiksten et al., 2005), but in prostate cancer high TATI expression is associated with aggressive disease (Paju et al., 2007).

Acute phase reaction. PSTI has been suggested to be an acute-phase protein and to be induced by inflammatory cytokines (Yasuda et al., 1990). The PSTI levels in serum increase in connection with severe inflammation, tissue destruction and major surgery (Lasson et al., 1986, Matsuda et al., 1985, Ogawa et al., 1985, Ogawa et al., 1988). PSTI-production in pancreatic acinar cells is not regulated by the acute-phase process, as suggested by analyzing PSTI, trypsinogen- 1 and $\alpha_{1}$-antichymotrypsin, another acute-phase reactant, in plasma and pancreatic juice after partial pancreatic resection (Jonsson et al., 1996).

In response to inflammatory cytokines, the liver produces several acute-phase proteins that are proteinase inhibitors. There is some evidence indicating that the liver might also be a source of PSTI in acute-phase reactions in humans. In cultured human hepatoblastoma cells, PSTI production is stimulated by IL-6 (Yasuda et al., 1990) and an IL-6-responsive element has been identified in the PSTI gene (Yasuda et al., 1993). Furthermore, PSTI is produced by hepatocellular cancer cells (Ohmachi et al., 1993) and the secretion of PSTI by human hepatocellular cancer cell line is substantially increased in the presence of cytokine-producing mononuclear white blood cells (Jonsson et al., 1996). Acute-phase proteins are thought to prevent non-specific tissue damage caused by proteinases released from activated immune and phagocytic cells (Roberts et al., 1995).

\section{Polyamines}

Polyamines, like spermidine and spermine, are needed for normal cellular growth and differentiation (Nitta et al., 2002). Exocrine pancreas has the highest spermidine concentration in the mammalian body, and it is thought to be related to the high rate of protein synthesis in this tissue. Activated polyamine catabolism in transgenic rats results in severe acute pancreatitis (Alhonen et al., 2000) and is associated with intracellular trypsinogen activation (Hyvonen et al., 2006). In the pancreas, polyamines have been localized in zymogen granules. Thus, it is possible that polyamines directly inhibit proteinase activity, and that their depletion thus would result in a direct activation of proteolytic enzymes (Hyvonen et al., 2006).

\section{Pancreatitis}

In the normal pancreas, the hazardous effects of proteinase activity are controlled by regulated expression and secretion, storage of zymogens within membrane-bound granules, 
regulated activation of the proenzymes, specific degradation and autolysis of the active proteinases, inhibition of their proteolytic activity, and controlled lysosomal degradation and autodegradation of digestive enzymes of damaged cells (Logsdon, 2001). However, pancreatitis is a necrotic and inflammatory process of the pancreas, where, with the exception of infectious pancreatitis, premature activation of trypsinogen and other digesitive pancreatic zymogens within or near the pancreas start digesting the pancreas itself (Kloppel and Maillet, 1993, Kloppel, 2007). Pathophysiologically, autodigestion and inflammation may be caused by either increased proteolytic activity or decreased proteinase inhibition.

Pancreatitis can be acquired or hereditary, acute or chronic (Kloppel and Maillet, 1993). Ethanol abuse and gallstones account for about $80 \%$ of acute pancreatitis cases (Le Moine et al., 1994, Lee et al., 1992). Chronic pancreatitis is usually caused by many years of alcohol abuse, ductal obstruction, exposure to cigarette smoke or volatile hydrocarbons, or can be autoimmune or hereditary (Chari, 2007, McNamee et al., 1994, Talamini et al., 1996).

Especially in acute pancreatitis (I) (Borgström and Andren-Sandberg, 1995, Kimland et al., 1989, Petersson et al., 1999), but also in pancreatic cancer, chronic alcoholism and chronic pancreatitis (Borgström and AndrenSandberg, 1995, Rinderknecht et al., 1979, Rinderknecht et al., 1985) the proportion of serum trypsinogen-1 and -2 immunoreactivity becomes reversed, suggesting nonparallel secretion of the trypsinogen isoforms in pancreatic disease. By using recombinantly produced trypsinogen-1 and -2, Kukor et al. (Kukor et al., 2003) demonstrated that the up-regulation of trypsinogen-2 in potential pathological conditions significantly limits trypsin generation. In conditions modeling those of pancreatic juice $\left(1 \mathrm{mmol} / \mathrm{L} \mathrm{Ca}^{2+}, \mathrm{pH}\right.$ 8 ), trypsin generation by autoactivation or enteropeptidase activation was not affected significantly by the ratio of the two isoforms due to faster autodegradation of trypsinogen-2 and trypsin-2. However, trypsin generation was markedly diminished under conditions that modeled cytoplasm or acidic vesicles (50 $\mu \mathrm{mol} / \mathrm{L} \mathrm{Ca}^{2+}, \mathrm{pH} 5$ ) by an increased ratio of trypsinogen-2, because acidic $\mathrm{pH}$ inhibited activation of trypsinogen-2, whereas it stimulated autoactivation of trypsinogen-1. This suggests that, as a defensive mechanism, acinar cells increase secretion of trypsinogen-2 in pancreatic diseases, thereby decreasing the chance for premature trypsinogen activation inside the pancreas, while maintaining acceptable trypsin function in the duodenum (Kukor et al., 2003).

\section{Hereditary pancreatitis}

Hereditary pancreatitis (HP) is caused by mutation(s) inducing premature intracellular activation of proteolytic enzymes, especially trypsin. The phenotypic features of hereditary pancreatitis include autosomal dominant inheritance, high penetrance $(80 \%)$, intermittent attacks of acute pancreatitis usually beginning in childhood, and frequent progression of the disease to chronic pancreatitis (Gorry et al., 1997). Patients with hereditary pancreatitis, especially those with a paternal inheritance pattern, have a high risk of developing pancreatic cancer several decades after the onset of pancreatitis (Lowenfels et al., 1997).

A relationship between the onset of pancreatitis and a mutation in the trypsinogen-1 gene was initially reported in 1996 (Whitcomb et al., 1996). Since then, several mutations in the trypsinogen-1 (PRSS1), PSTI (SPINK1), and cystic fibrosis transmembrane conductance regulator (CFTR) genes have been found to be associated with chronic pancreatitis (Keiles and Kammesheidt, 2006). An up-todate database of published PRSS1, PRSS2 and SPINK1 variants can be found at www.unileipzig.de/pancreasmutation.

Mutations in the PRSS1 gene. The AA substitutions in trypsinogen-1 are located in the activation peptide, the N-terminal part of 
trypsin, and in the longest peptide segment not stabilized by disulfide bonds between Cys64 and Cys139, which also encompasses the calcium-binding loop. The mutations appear to be associated with enhanced activation (Chen et al., 2003a, Feréc et al., 1999, Gorry et al., 1997, Pfutzer et al., 2002, Sahin-Tóth and Tóth, 2000, Sahin-Tóth, 2000, Sahin-Tóth, 2001, Simon et al., 2002, Teich et al., 2000, Teich et al., 2004, Whitcomb, 1999), inhibition of autolysis, or enhanced stabilization (Le Maréchal et al., 2001, Pfutzer et al., 2002, Sahin-Tóth, 2001, Simon et al., 2002, Whitcomb et al., 1996).

The most frequent mutation in HP worldwide is Arg122His, which eliminates the autolysis site of trypsin- 1 and alters autoactivation and autodegradation of trypsinogen-1 (Simon et al., 2002). Unlike all other known trypsinogens, human trypsinogen-1 contains Asn at position 29 . With the exception of human trypsinogen-2 that has Ile at position 29, all other mammalian trypsinogens contain Thr29 (Rypniewski et al., 1994). The second most frequent HPassociated mutation is Asn29Ile in human trypsinogen-1 (Gorry et al., 1997). Other mutations of Asn29 have also been shown to affect autoactivation (Sahin-Tóth, 2000). Chymotrypsin $\mathrm{C}$-mediated processing of the trypsinogen-1 activation peptide is increased 4-fold by the mutation Ala16Val, resulting in accelerated trypsinogen activation in vitro (Nemoda and Sahin-Tóth, 2006).

A novel mechanism underlying HP was suggested by Teich et al. (Teich et al., 2004). The activation of trypsinogen-2 by mutated Glu79Lys-trypsin-1 was increased two-fold, and HP could thus be caused by increased transactivation of trypsinogen-2 by mutated trypsin-1. Furthermore, triplication of a $\sim 605$ kb gene segment containing the PRSS1 gene on chromosome 7 seems to result in increased trypsin expression through a gene dosage effect causing HP (Le Maréchal et al., 2006).

Mutations that protect against pancreatitis are very rare but have been reported. Mutations Tyr37X and IVS2+1G $>$ A that result in non- functional product of the PRSS1 gene were found in two of 55 alcoholics without chronic pancreatitis, respectively (Chen et al., 2003b). Furthermore, mutation Gly191Arg results in degradation-sensitive trypsinogen-2 (Witt et al., 2006).

It has been suggested that gene conversion is a likely cause of PRSS1 missense mutations associated with HP (Chen and Feréc, 2000a, Chen and Feréc, 2000b). Gene conversion is a process where a functional gene is converted into a mutant one by unidirectional transfer of genetic information from a homologous, non-functional donor gene to the functional acceptor gene (Baltimore, 1981, Chen et al., 2007). The genes $\mathrm{T} 4$ to $\mathrm{T} 8$ are organized in tandem repeats and share $91 \%$ overall sequence homology (Rowen et al., 1996), which render them prone to gene conversion events. The presence of several donor sequences in genes T6, T7, T8 and T9 for Arg 122His, Asn29Ile and Ala16Val mutations, respectively, is strongly suggestive of these mutations being caused by gene conversion events. Furthermore, chilike and palindromic sequences are frequently observed in the vicinity of potentially converted gene fragments (Collier et al., 1993, Giordano et al., 1997, Patrinos et al., 1998). Such sequences are also found in the 3' and/or 5' boundaries of the Arg122His, Asn29Ile and Ala16Val mutations (Chen and Feréc, 2000a, Chen and Feréc, 2000b).

Mutations in the SPINK1 gene. Mutations and polymorphisms in the SPINK1 gene are also associated with HP. These include singlenucleotide substitutions, microinsertions/ deletions (Chen et al., 2000, Kiraly et al., 2007, Le Maréchal et al., 2004, Pfutzer et al., 2000, Witt et al., 2000) and a large 1336 bp deletion involving the promoter region and exon 1 of SPINK1 (Masson et al., 2006).

The Asn34Ser mutation (Witt et al., 2000) is the most common HP-associated variant in the PSTI gene. Asn34 is located close to Lys18, the target $\mathrm{P} 1$ residue of trypsin, and the mutation has been suggested to lead to decreased inhibitory capacity of PSTI by affecting the 
conformation of the active site (Witt et al., 2000). On the other hand, biochemical and surface-plasmon-resonance (SPR) analysis of recombinant Asn34Ser mutant and wild type PSTI showed no difference in binding (Hirota et al., 2003). Thus, the Asn34Ser mutation has been suggested to act more like a disease susceptibility factor, possibly by lowering the threshold for pancreatitis caused by other genetic or environmental factors (Masson et al., 2006, Pfutzer et al., 2000, Schneider et al., 2002). Interestingly, the Asn 34 Ser mutation always co-segregates with intronic mutations and altered splicing has been suggested to underlie the predisposition to HP (Kuwata et al., 2002).

The Met1Thr mutation eliminates the start codon of PSTI leading to an overall loss of PSTI expression (Witt et al., 2000). The Arg67Cys mutation has been suggested to cause massive conformational alterations in the protein, probably by a novel intra- or intermolecular disulfide bond, as the mutant recombinant PSTI isoforms lost their reactivity with an anti-PSTI (wild type) antibody (Hirota et al., 2003). Signal peptide variants that have been demonstrated to impair secretion of PSTI and destine the inhibitor for rapid intracellular degradation are also associated with HP (Kiraly et al., 2007).

Cystic fibrosis transmembrane conductance regulator gene mutations. Mutations in the cystic fibrosis transmembrane conductance regulator gene (CFTR), located on chromosome 7q31 (Riordan et al., 1989), lead to exocrine glandular dysfunction and cystic fibrosis, but are also associated with chronic pancreatitis (Audrezet et al., 2002, Sharer et al., 1998). The first organ to be affected in phenotypical severe CF is the exocrine pancreas (Schwiebert et al., 1998). In a cohort of 134 patients with chronic pancreatitis Sharer et al. (Sharer et al., 1998) found - and the results have been confirmed by others (Cohn et al., 1998, Ockenga et al., 2000) - that the frequency of CFTR mutation was higher than expected. Thus, mutations of the CFTR gene are a risk factor for chronic pancreatitis.
The CFTR protein is present at high levels in intralobular and proximal ductular epithelia of the pancreas and at low levels in acinar cells. There it maintains the solubility of the secreted enzymes by mediation of the secretion of bicarbonate-rich alkaline fluid (Shumaker et al., 1999). Impaired solubility of pancreatic juice may thus be responsible for the increased risk of pancreatitis. Decreased activity of the mutated CFTR protein has also been suggested to interact with mutations in PSTI or trypsinogen-1 gene (Hirota et al., 2006b).

$H P$ and acinar cell viability. The role of trypsinogen-1 in pancreatitis has been studied by the expression of wild type active trypsin-1, trypsinogen-1 and trypsinogen-1 bearing HPassociated mutations in the pancreatic acinar cell line AR4-2J (Gaiser et al., 2005). AR4-2J cell viability was reduced dose-dependently by transfection with a vector coding for functional trypsin-1, trypsinogen mutants Ala16Val, Asp22Gly, Lys23Arg and Arg122His. Wild type trypsinogen-1 had no effect on cell viability. Caspase-3 activity was shown to be significantly higher in cells expressing active trypsin-1 or the Arg122His trypsinogen-1 than in cells expressing wild type trypsinogen-1. Furthermore, caspase- 3 activity was reduced in the presence of trypsin inhibitor. These findings suggest that expression of HPassociated mutations result in a stress strong enough to induce apoptosis in AR4-2J cells probably due to intracellular occurence of active trypsin. Thus, the sensibility of acinar cells to intracellular trypsin activity leading to apoptosis might have a protective effect in the pancreas (Gaiser et al., 2005).

\section{Clinical value of trypsinogen determinations}

In serum of healthy subjects, the average concentration of trypsinogen-1 has been reported to be 15 to $26 \mu \mathrm{g} / \mathrm{L}$ (I) (Borgström and Ohlsson, 1976, Florholmen et al., 1984b, Geokas et al., 1979) and that of trypsinogen-2 5.5 to $17 \mu \mathrm{g} / \mathrm{L}$ (I) (Largman et al., 1978). 
Cystic fibrosis. The exocrine pancreas is affected in cystic fibrosis (CF), a disease caused by mutations in a chloride channel encoded by cystic fibrosis transmembrane conductance regulator gene (CFTR) (Aleksandrov et al., 2007, Riordan et al., 1989). Pancreatic damage begins in utero, which can be identified in neonates on the basis of elevated blood concentrations of pancreatic enzymes, especially trypsinogen (Sharer et al., 1998 and references therein). Transiently elevated serum levels of immunoreactive trypsinogen (IRT) have been found to be associated with the presentation of typical as well as atypical CF (Castellani et al., 1997). Determination of serum IRT in neonates is used as a test for screening for $\mathrm{CF}$ in many countries (Rock et al., 2005). Another test for CF is the sweat chloride test. A positive result with IRT and/ or sweat chloride test is confirmed by gene mutation analysis (Spence et al., 1993).

Acute pancreatitis. Trypsinogen, trypsinogen activation peptide (TAP) and trypsin-API complexes in body fluids have been found to be increased in acute pancreatitis and can thus be used as diagnostic markers (I) (Borgström and Ohlsson, 1978, Borgström et al., 2002, Brodrick et al., 1979, Dubick et al., 1987, Elias et al., 1977, Florholmen et al., 1984b, Geokas et al., 1979, Gudgeon et al., 1990, Hedström et al., 1994, Hedström et al., 1996c, Hedström et al., 1996d, Petersson and Borgström, 2006, Petersson et al., 1999, Sainio et al., 1996, Tenner et al., 1997). Commercial radioimmunoassays, enzyme immunoassays and immunofluorometric assays for trypsinogen as well as TAP ELISA assay are available. A rapid dipstick screening test for pancreatitis, based on immunochromatographic measurement of urinary trypsinogen-2, has been developed (Hedström et al., 1996b, Kemppainen et al., 1997) and shown to detect acute pancreatitis more accurately than routinely used quantitative serum or urinary amylase determinations. However, serum amylase, often supplemented with serum lipase has remained the cornerstone laboratory test for diagnosis of acute pancreatitis in hospitals.
Allograft rejection and malnutrition. Serum trypsinogen-2 is shown to be accurate and sensitive diagnostic marker for rejection and inflammation occurring in the pancreatic allograft following pancreaskidney transplantation (Douzdjian et al., 1994, Lieberman et al., 1997, Perkal et al., 1992). Co-monitoring serum amylase and trypsinogen-2 increased the specificity and diagnostic accuracy of the biochemical tests (Lieberman et al., 1997). Furthermore, increased levels of serum trypsinogen-1 have been reported in acutely malnourished infants and children (Durie et al., 1985). Improvement in nutritional status reverted trypsinogen-1 levels to normal.

Malignancies. Trypsinogens are associated with several malignancies and they could be used as diagnostic and prognostic factors for some cancers. However, the use of these tests is very limited. TAT- 2 complexed with API has been shown to be a strong prognostic marker in advanced epithelial ovarian cancer (Paju et al., 2004). Immunohistochemically detected trypsin has been shown to correlate with disease recurrence and poor prognosis in human colorectal cancer (Yamamoto et al., 2003) and esophageal squamous cell carcinoma (Yamamoto et al., 2001). In nonsmall cell lung cancer (NSCLC) up-regulation of trypsinogen IVb (PRSS3) and trypsinogen C (TRY6) gene expression are predictors of distant metastasis and survival as revealed by microarray analysis (Diederichs et al., 2004).

Serum trypsinogen-2 has high accuracy in differentiating between cholangiocarcinoma and primary sclerosing cholangitis (Lempinen et al., 2007). Thus, it is a useful marker diagnosing patients with cholangiocarcinoma, and it is superior to serum tumor markers CA 19-9 and CEA. 


\section{Post-translational modification of proteins}

The explosion of genetic information has increased our knowledge of living systems enormously. However, the structure of a mature protein is not dependent solely upon its gene, but also on post-translational modifications (PTMs). Chemical, biochemical, and enzymatic PTM of proteins to specific amino acid residues are common as over 200 variant amino acid residues have been detected (Creighton, 1984). They include disulfide bridge formation, glycosylation, proteolysis, phosphorylation, acylation, adenylation, farnesylation, ubiquitination, sulfation, amidation, oxidation, methylation, nitration, citrullination, isoprenylation, and palmitoylation, among others. These modifications affect the properties of proteins in many ways, i.e. activity, lifespan and protein-protein interactions (Hochrainer and Lipp, 2007, Li and Shang, 2007, Omary et al., 2006, Vader et al., 2006, Vervoorts et al., 2006, van der Horst and Burgering, 2007).

\section{Tyrosine $\boldsymbol{O}$-sulfation}

Tyrosylprotein sulfotransferase. Sulfate trioxide $\left(\mathrm{SO}_{3}\right)$ may be covalently bound to the hydroxyl group on the side-chain of tyrosine and each sulfate moiety increases the molecular mass of the protein by $79.957 \mathrm{Da}$ (Kehoe and Bertozzi, 2000). Protein tyrosine $O$-sulfation was first observed by Bettelheim in bovine fibrinopeptide B in 1954 (Bettelheim, 1954). Later, it was shown to be a ubiquitous protein modification (Huttner, 1982) mediated by tyrosylprotein sulfotransferase (TPST, EC 2.8.2.20) (Huttner, 1987, Lee and Huttner, 1983). TPST catalyzes the transfer of sulfate from the universal sulfate donor 3'-phosphadenosine 5'phosphosulfate (PAPS) to the hydroxyl group of tyrosine residues of proteins to form a tyrosine $\mathrm{O}^{4}$-sulfate ester and 3',5'-ADP (Lee and Huttner, 1983). TPST is an integral membrane glycoprotein present in two forms (TPST-1 and TPST-2) in the trans Golgi network, and the two forms are coexpressed in many species, tissues and cell lines throughout the plant and animal kingdom examined so far (Baeuerle and Huttner, 1987, Beisswanger et al., 1998, Huttner, 1987, Lee and Huttner, 1985, Moore, 2003, Niehrs and Huttner, 1990, Ouyang and Moore, 1998, Ouyang et al., 1998, Ouyang et al., 2002, Vargas et al., 1985, William et al., 1997, William et al., 1997).

Predicted tyrosine sulfation sites. The Golgi localization and the luminal active site orientation of TPST-1 and -2 predict that tyrosine $O$-sulfation occurs only on proteins that transit through the trans Golgi network and there is no evidence of violation of this rule (Moore, 2003). Not only secreted proteins but also membrane-bound proteins are equally likely to be sulfated (Hille and Huttner, 1990, Hille et al., 1990). There is no sequon for tyrosine $O$-sulfation per se, but consensus features predicting tyrosine sulfation have been proposed.

First, the presence of acidic amino acids like aspartic or glutamic acid at position -1 and at least two more acidic residues present between positions -5 and +5 of the sulfated tyrosine occur frequently. Secondly, the presence of turn-inducing amino acids within positions -7 to -2 and +1 to +7 of the tyrosine sulfate residues seem to form a favorable secondary structure for the recognition of substrate proteins by TPST. Finally, no identified tyrosine sulfation site contains a PTM causing steric hindrance like disulfide bonds or N-glycosylation near the tyrosinesulfate residue (Hortin et al., 1986, Huttner, 1987, Niehrs and Huttner, 1990, Niehrs et al., 1990). Later, data from sitedirected mutagenesis of human progastrin in vivo (Bundgaard et al., 1997) show that basic residues around sulfation site are allowed, though not in position -1 .

Frequency. A software tool called Sulfinator for prediction of tyrosine sulfation sites in protein sequences is accessible on the ExPASy server at the URL http://www.expasy.org/tools/ sulfinator/ (Monigatti et al., 2002). Scanning with Sulfinator of proteins from various species that according to SWISS-PROT pass through the secretory pathway suggest that one third of 
proteins that enter the secretory pathway may contain on average two tyrosine sulfation sites per protein (Monigatti et al., 2002). Another estimation is that $7 \%$ of mammalian proteins are tyrosine sulfated (Moore, 2003). According to an in vivo labeling study of Drosophila melanogaster with inorganic ${ }^{35} \mathrm{SO}_{4}$ as much as $1 \%$ of the tyrosine residues of the proteins in an organism can be sulfated (Baeuerle and Huttner, 1985).

Regulation of tyrosine O-sulfation. The regulation of tyrosine $O$-sulfation is not known. The fact that tyrosine sulfation is poorly reversible or even irreversible in vivo and in vitro suggests that tyrosine $O$-sulfation is not modulated by the sulfatases (Dodgson et al., 1959, Dodgson et al., 1961, Jones et al., 1963, Tallan et al., 1955). Sardinello and coworkers determined by a genomic approach the complete catalog of human sulfatases, which comprises 17 members, but no extracellular sulfotyrosylprotein sulfatase was identified (Sardiello et al., 2005). Tyrosine phosphorylation, which is chemically and structurally a close relative PTM to tyrosine sulfation, is mediated by a rich array of kinases and phosphatases and is involved in multiple signaling and regulatory functions in the cells (Craven et al., 2003, Wang et al., 2003). The small number of TPSTs and the apparent absence of sulfotyrosylprotein sulfatase suggest that protein TPST isoforms are expressed in a cell-specific manner (Bundgaard et al., 1997). However, evidence for transcriptional regulation of the TPST-1 and TPST-2 genes is very limited (Moore, 2003).

\section{Effects of tyrosine-sulfation}

Known human tyrosine-sulfated proteins include adhesion molecules, G-protein coupled receptors, coagulation factors, serpins, extracellular matrix proteins, hormones, enzymes and others (Moore, 2003). Posttranslational tyrosine $O$-sulfation of proteins may affect protein-protein interactions involved in leukocyte adhesion (Fong et al., 2002, Kehoe and Bertozzi, 2000), hemostasis
(Leyte et al., 1991, Michnick et al., 1994, Pittman et al., 1994), chemokine signaling (Kehoe and Bertozzi, 2000), intracellular protein transport and secretion (Friederich et al., 1988), prohormone processing (Bundgaard et al., 1995, Huttner, 1987), receptor-ligand binding (Choe et al., 2005, Costagliola et al., 2002, Gao et al., 2003, Wilkins et al., 1995) and it may influence the biological activity (Brand et al., Dorfman et al., 2006, Hortin et al., 1989) and half-life of proteins (Huttner, 1987).

The HIV-1 envelope glycoprotein has been reported to use sulfotyrosines of the chemokine receptor CCR5 to enter cells that express this obligate coreceptor (Farzan et al., 2002). Likewise, the Duffy antigen/receptor for chemokines (DARC) is necessary for entry of Plasmodium vivax malaria into maturing red blood cells, and a sulfotyrosine at the DARC amino terminus mediates its association with the $P$. vivax Duffy-binding protein (Choe et al., 2005). It is suggested that sulfotyrosines may be especially adept at binding diverse proteins with high affinity since the sulfate group distinctively modifies the electronic properties of the phenyl ring of the tyrosine, providing abundant, highly polarizable electrons. Therefore, the sulfate group provides some level of specificity but can also accommodate subtly different microenvironments (Choe and Farzan, 2006).

To assess the role of tyrosine sulfation in vivo, Tpst 1 and Tpst 2 knock-out mice have been generated by targeted disruption of the Tpst 1 and Tpst2 genes (Borghei et al., 2006, Ouyang et al., 2002). Disruption of either the Tpst1 or Tpst 2 gene decreased postnatal growth. Maternal TPST-1 deficiency also reduced the litter size due to fetal loss and increased perinatal mortality. TPST- 2 deficient male, but not female mice, were infertile. It seems that protein(s) required for normal male reproductive function must undergo tyrosine $O$-sulfation to function normally and that these proteins can be sulfated in vivo in the absence of TPST-1 but not TPST-2. High affinity and specific anti-sulfotyrosine MAbs have recently 
been generated and this will facilitate further investigation and identification of tyrosinesulfated proteins (Hoffhines et al., 2006, Kehoe et al., 2006).

\section{Post-translational modification of pancreatic trypsinogens}

The first evidence for sulfation of pancreatic trypsinogen-1 and -2 came from twodimensional isoelectric focusing/sodium dodecyl sulfate gel electrophoresis. Incorporation of ${ }^{35} \mathrm{SO}_{4}$ into trypsinogen of pancreatic tissue slices was demonstrated by fluorography of tissue homogenates separated by the two-dimensional gel procedure. Results from acid treatment of the homogenates suggested that the sulfate moiety was covalently attached to tyrosine residue (Scheele et al., 1981). Preliminary ESI MS data from Szilagyi and colleagues (Szilagyi et al., 2001) suggest that the modifying group at Tyr154 in trypsinogen-1 is sulfate and not phosphate as based on the crystal structure study of Gaboriaud and colleagues (Gaboriaud et al., 1996). Later, sulfated tyrosine residues from purified trypsinogen isoenzymes, subjected to alkaline hydrolysis, have been identified by thin layer chromatography (Sahin-Tóth et al., 2006). Furthermore, incorporation of ${ }^{35} \mathrm{SO}_{4}$ into human trypsinogen-1 transiently expressed by human embryonic kidney 239T cells was demonstrated. Mutation of Tyr154 to Phe abolished radioactive sulfate incorporation confirming that Tyr154 is the site of sulfation in trypsinogen- 1 .

When comparing the sulfated pancreatic trypsinogen-1 and its nonsulfated recombinant form, it was found that the sulfated trypsinogen-1 underwent faster autoactivation. This suggests that tyrosine sulfation might enhance intestinal digestive zymogen activation in humans (Sahin-Tóth et al., 2006). The amidolytic and esterolytic activity of modified and non-modified trypsin-1 are essentially identical, but sulfated trypsin- 1 is slightly better inhibited by PSTI (Szilagyi et al., 2001). The finding that mRNA expression of the TPST-2 isoform is drastically higher in the pancreas than in any other tissues examined (Ouyang and Moore, 1998) is thought to explain the high stoichiometry of human pancreatic trypsinogen-1 and -2 sulfation. 


\section{Aims of the present study}

Tumor-associated trypsin inhibitor (TATI) has been isolated from urine of an ovarian cancer patient (Stenman et al., 1982) and shown to be identical to pancreatic secretory trypsin inhibitor (PSTI) (Huhtala et al., 1982). In search for a target protease for TATI, two trypsinogen isoenzymes were characterized in cyst fluid of mucinous ovarian tumors (Koivunen et al., 1989). The N-terminal amino acid sequences of these tumor-associated isoenzymes corresponded to those of pancreatic trypsinogen-1 and -2 respectively. However, the isoenzymes had different specificities for $p$-nitroanilide substrates, responded differently to various protease inhibitors and had different isoelectric points from those of trypsinogen-1 and -2 . Therefore, they were named tumorassociated trypsinogen-1 and trypsinogen-2 (TAT-1 and TAT-2) (Koivunen et al., 1989).

The first aim of the present study was the preparation of specific monoclonal antibodies to trypsinogen isoenzymes, the development of quantitative immunoassays for trypsinogen-1 and -2 , and the purification, identification and characterization of pancreatic and tumor-associated trypsinogen isoenzymes by various chromatographic and immunologic techniques.

There have been contradictory reports about post-translational modification of pancreatic trypsinogen-1. The molecular weight of human pancreatic trypsinogen-1 has been determined by mass spectrometry to be 80 Da higher than the theoretical mass deduced from the polypeptide sequence (Gaboriaud et al., 1996, Szilagyi et al., 2001). Based on the X-ray electron density map, the observed mass difference was attributed to phosphorylation at tyrosine residue 154 (Gaboriaud et al., 1996). However, incorporation of ${ }^{35} \mathrm{SO}_{4}$ to trypsinogen-1 and -2 in pancreatic tissue culture (Scheele et al., 1981) on one hand, and alkaline hydrolysis of purified trypsinogens and subsequent separation of modified tyrosine residues by thin layer chromatography on the other hand, has revealed that both human trypsinogen-1 and -2 contain tyrosine sulfate (Sahin-Tóth et al., 2006). Furthermore, trypsinogen-1 expressed in human embryonic kidney 293T cells has been shown to incorporate ${ }^{35} \mathrm{SO}_{4}$ into the secreted trypsinogen and mutation of Tyr154 to Phe was shown to abolish the incorporation (Sahin-Tóth et al., 2006).

The second aim of this study was to characterize by mass spectrometry the chemical modification underlying the observed differences in isoelectric point, substrate binding and inhibitor specificity between pancreatic and tumor-associated trypinogen-1 and -2 , respectively. 


\section{Materials and methods}

The materials and methods are described in detail in the original papers (I-IV).

\section{Samples, patients and cell lines (I- IV)}

The ethical committee of Helsinki University Central Hospital, Finland, has approved the use of human samples in this study. Cyst fluid of ovarian tumors was obtained in connection with surgical removal of the tumors. Pancreatic fluid was collected by duodenal catheterization of patients who were examined because of biliary or pancreatic diseases. Benzamidine and aprotinin were added to the pancreatic fluid to a final concentration of $10 \mathrm{mmol} / \mathrm{L}$ and $10 \mu \mathrm{g} / \mathrm{L}$, respectively. Serum samples from patients with pancreatitis and patients who had undergone total pancreatoduodenectomy were kindly provided by Dr. Tom Schröder, Helsinki University Central Hospital, Finland. Serum samples from healthy individuals were collected from the laboratory staff and from women with benign cysts, infertility or pregnancy, and were used to calculate the reference range for trypsinogen- 1 and -2 . Preovulatory follicular fluid was obtained from patients participating in an in vitro fertilization. All samples were stored aliquoted at $-20^{\circ} \mathrm{C}$ or $-80^{\circ} \mathrm{C}$. The colon adenocarcinoma cell line, COLO 205, was from American Type Culture Collections, and was cultured according to the guidelines provided.

\section{Monoclonal antibodies (I)}

Monoclonal antibodies, or MAbs, were obtained by immunizing BALB/c mice (from the Zentralinstitut für Versuchstiersucht, Hannover, Germany) intraperitoneally with $50 \mu \mathrm{g}$ of TAT containing both isoenzymes emulsified in Freund's complete (first injection) or incomplete adjuvant three times at 2-week intervals. A booster of $10 \mu \mathrm{g}$ TAT in saline solution was given intravenously four days before fusion. The fusion was performed as described by Köhler and Milstein (Köhler and Milstein, 1975). Antibodies produced by the hybridomas were screened by a TR-IFMA. Hybridomas secreting MAb to trypsinogen-isoenzymes were selected, cloned and expanded. Three MAbs designated 2F3, 3E8 and 6D11 reacted predominantly with trypsinogen-1 and two MAbs, 14D4 and $14 \mathrm{~F} 10$, reacted with trypsinogen- 2 .

For the production of large amounts of MAbs in ascites fluid BALB/c mice were primed intraperitoneally with $0.5 \mathrm{~mL}$ pristane $(96 \%$ 2,6,10,14-tetramethylpentadecane, AldrichChemie) one week before the injection of 0.4 - $1 \times 10^{6}$ hybridoma cells (Hoogenraad and Wraight, 1986). Ascites fluid was centrifuged and the immunoglobulin fraction from ascites fluid was precipitated with $\mathrm{Na}_{2} \mathrm{SO}_{4}$ at final concentration of $180 \mathrm{~g} / \mathrm{L}$. After washing the precipitate twice it was dissolved in $\mathrm{Na}_{2} \mathrm{CO}_{3}$ (100 mmol/L, pH 9.0) and stored frozen at $-20^{\circ} \mathrm{C}$. Alternatively, antibody-producing hybridomas were cultured in INTEGRA CL 1000 flasks (Integra Biosciences), and the MAbs were purified from the culture supernatant by protein $\mathrm{G}$ or protein A affinity chromatography (MAbTrap ${ }^{\mathrm{TM}}$ or Protein G Sepharose 4 fast flow from GE Healthcare Bio-Sciences or PROSEP-A from Millipore) and MAbs were eluted according to the instructions of the respective manufacturer.

The subclass of the MAbs was determined by immunodiffusion (Ouchterlony, 1958) with specific antibodies from Nordic Immunological Laboratories. All MAbs were of the immunoglobulin G1 isotype.

\section{Time-resolved immunofluorometric assays (I)}

MAb production by hybridoma cells was detected by a sandwich TR-IFMA employing 
polyclonal rabbit antiserum to human pancreatic trypsin (Koivunen et al., 1989) immobilized onto microtiter wells, to bind partially purified trypsinogen-1 or -2 to the immobilized antibody. The trypsinogen isoenzyme reactive MAbs were detected by rabbit anti-mouse immunoglobulins (Dako) labeled with europium $(\mathrm{Eu})$.

Two TR-IFMAs were developed to recognize trypsinogen-1 and -2, respectively. Each assay is based on the combination of two specific antibodies, catcher antibody immobilized onto microtiter wells and tracer antibody labeled with Eu chelate.

Catcher MAbs were immobilized onto 200 $\mu \mathrm{L}$ polystyrene microtiter wells by incubating $200 \mu \mathrm{l}$ corresponding to $2 \mu \mathrm{g}$ of MAb in $\mathrm{Na}_{2} \mathrm{CO}_{3}(100 \mathrm{mmol} / \mathrm{L}, \mathrm{pH} 9.0)$ overnight at $+4^{\circ} \mathrm{C}$. To block non-specific adsorption $1 \%$ bovine serum albumin in TBS $(50 \mathrm{mmol} / \mathrm{L}$ Tris- $\mathrm{HCl}$ buffer, $\mathrm{pH} 7.4$ containing $9 \mathrm{~g} / \mathrm{L} \mathrm{NaCl}$ and $0.5 \mathrm{~g} / \mathrm{L} \mathrm{NaN}_{3}$ ) was added to the wells and left overnight at $+4^{\circ} \mathrm{C}$. The BSA-solution was then discarded and the wells were stored in a moist atmosphere at $+4^{\circ} \mathrm{C}$.

Tracer MAbs were labeled with isothiocyanato-phenyldiethylenetriamine$N^{1}, N^{2}, N^{3}, N^{4}$-tetraacetate chelated with europium(III) (Hemmilä et al., 1984) with a 100 -fold molar excess of the chelate. After incubation overnight at $+4^{\circ} \mathrm{C}$ unbound chelate was separated from the labeled MAb by gel chromatography on a $1 \times 15 \mathrm{~cm}$ column of Sephacryl S200 HR (Pharmacia Biotech) using TBS as eluent. Further purification was achieved by hydrophobic interaction chromatography on a $2 \mathrm{~mL}$ phenyl-Sepharose column (Pharmacia, Uppsala, Sweden) equilibrated with TBS. After application of the labeled MAb, the column was eluted with $10 \mathrm{~mL}$ of TBS at hydrostatic pressure. The nonadsorbed fraction was collected, stored at $+4^{\circ} \mathrm{C}$ and used as tracer MAb in the TRIFMA.

All possible combinations of MAbs were tested as catcher and tracer for the optimal assay for trypsinogen-1 and -2 . For assay of trypsinogen-1, combinations of MAbs designated $6 \mathrm{D} 11$ as catcher and either 2F3 or $3 \mathrm{E} 8$ as the tracer were selected. For trypsinogen-2 MAbs 14F10 and 14D4 have been used as either catcher or tracer.

TR-IFMA for trypsinogen-1 and -2 is performed as described (I). Briefly, $25 \mu \mathrm{L}$ of standard or sample along with $200 \mu \mathrm{L}$ buffer was incubated in the MAb-coated wells for one hour at room temperature with constant shaking. The wells were washed and $50 \mathrm{ng}$ of Eu-labeled MAb in $200 \mu \mathrm{L}$ was added and incubated for 30 minutes as above. After washing the wells $200 \mu \mathrm{L}$ of enhancement solution was added and the mixture was shaken for 5 minutes. Fluorescence was then measured for one second per well in an LKB 1230 Arcus Fluorometer.

TR-IFMA for trypsinogen-1 was calibrated by using PMSF-inhibited pancreatic trypsin-1 covering the concentration range 0.24 to 250 $\mu \mathrm{g} / \mathrm{L}$. Today, TAT- 1 recombinant protein is used as standard. TR-IFMA for trypsinogen-2 was calibrated by using the zymogen form of TAT-2 purified from culture medium of COLO 205 cells. The standard curve covered the range 0.97 to $495 \mu \mathrm{g} / \mathrm{L}$. As assay calibrators we use secondary standards diluted from ovarian cyst fluid.

Sensitivity of the TR-IFMAs for trypsinogen-1 and -2 assay, respectively, was calculated from the mean fluorescence signal of a zero sample (assay buffer, $n=20$ ) plus two standard deviations. The intra- and interassay variation of the assays was calculated from the repetitive results of control samples (mucinous ovarian cyst fluids diluted in assay buffer) stored at $-20^{\circ} \mathrm{C}$. The cross-reaction of each isoenzyme in the assay for the other isoenzyme was confirmed by assaying trypsinogen-1 and -2 separated by anion exchange chromatography.

\section{Radioimmunoassays (I and II)}

RIA kits for the determination of trypsin-like 
immunoreactivity were obtained from CIS and were used according to the instructions of the manufacturer. The assay employs rabbit antiserum to human trypsin-1, ${ }^{125}$ I labeled trypsin-1 as tracer, and is calibrated with trypsin-1 purified by Trasylol (aprotinin)affinity chromatography. The serum sample volume used in the assay was $100 \mu \mathrm{L}$.

RIA of TATI was performed employing a polyclonal rabbit antiserum and ${ }^{125}$ I labeled TATI as described (Stenman et al., 1982). Concentration of TATI in normal serum was reported to be 5 to $20 \mu \mathrm{g} / \mathrm{L}$.

\section{Characterization of trypsinogens by gel filtration chromatography (I and II)}

Serum samples $(0.5 \mathrm{~mL})$ were applied to a 1 x $35 \mathrm{~cm}$ Sephacryl S-200 column and eluted with TBS at a flow rate of $20 \mathrm{~mL} / \mathrm{hr}$ at $+4^{\circ} \mathrm{C}$. One $\mathrm{mL}$ fractions were collected and $100 \mu \mathrm{L}$ of a solution that contained $50 \mathrm{~g}$ bovine serum albumin, $5 \mathrm{~g}$ bovine immunoglobulin, 10 $\mathrm{mg}$ aprotinin and $1 \mathrm{~g}$ Tween-40 per liter was added to each fraction. Immunoreactivity of the fractions was determined by TR-IFMA for trypsinogen- 1 and -2 with a sample volume of $200 \mu \mathrm{L}$.

Gel filtration of hyperstimulated follicular fluid and filtered ovarian cyst fluid was performed on Superose 12 column (Pharmacia) in TBS at a flow rate of $0.5 \mathrm{~mL} /$ minute. The sample volume was $200 \mu \mathrm{L}$ and fraction size $400 \mu \mathrm{L}$.

The gel filtration columns were calibrated with albumin $67 \mathrm{kDa})$, ovalbumin (43 kDa), soybean trypsin inhibitor $(21 \mathrm{kDa})$ and aprotinin $(6 \mathrm{kDa})$.

\section{Purification of trypsinogen (I, II and IV)}

Trypsinogen from mucinous ovarian cyst fluid was purified by a combination of batch-wise anion exchange, immunoaffinity, and reversephase (RP) chromatography as described elsewhere (Koivunen et al., 1989). Briefly, cyst fluid was centrifuged and dialyzed against distilled water with a hollow fiber dialyzer. The dialyzed cyst fluid was mixed overnight with a strong anion exchanger, Q Sepharose (Pharmacia Biotech). After washing away unbound material, bound proteins were eluted with $1 \mathrm{~mol} / \mathrm{L} \mathrm{NaCl}$ and $1 \%$ isopropanol. Trypsinogen from human pancreatic juice or conditioned media from COLO 205 cells was purified by immunoaffinity chromatography after centrifugation and $\mathrm{pH}$ adjustment to 7.5. Before immunoaffinity chromatography, benzamidine, aprotinin and Brij 35 were added to final concentration of $10 \mathrm{mmol} / \mathrm{L}, 10 \mathrm{mg} / \mathrm{L}$ and $0.1 \%$, respectively, to all preparates.

Immunoaffinity columns were produced by coupling MAbs 3E8 for trypsinogen-1 and MAb 14F10 for trypsinogen-2 to $\mathrm{CNBr}$-activated Sepharose 4B (Pharmacia Biotech) according to the manufacturer's instructions. The starting material was pumped at a flow rate of 10 to $30 \mathrm{~mL} / \mathrm{h}$ through the two immunoaffinity columns connected in tandem. After sample application the columns were separated and washed. The bound fraction was eluted with $0.1 \%$ TFA containing $1 \mathrm{mmol} / \mathrm{L} \mathrm{CaCl}_{2}, 10$ $\mathrm{mmol} / \mathrm{L}$ benzamidine and $10 \mathrm{mg} / \mathrm{L}$ aprotinin, collected in one $\mathrm{mL}$ fractions, neutralized and assayed for trypsinogen immunoreactivity. Fractions containing trypsinogen were further purified by ion exchange or reverse-phase HPLC. All purification steps except HPLC were carried out at $+4^{\circ} \mathrm{C}$.

\section{Separation of trypsinogen iso- enzymes by anion exchange and RP HPLC (I, III and IV)}

Before anion exchange chromatography was performed, serum samples and cell culture media were diluted fivefold, and cyst fluids were diluted 10 - to 30 -fold with $50 \mathrm{mmol} / \mathrm{L}$ Tris-HCl buffer, $\mathrm{pH}$ 8,0 (buffer A). Five hundred $\mu \mathrm{L}$ of diluted sample was applied to a Mono Q HR 5/5 or Resource Q anion exchange column (Pharmacia Biotech) equilibrated with buffer $\mathrm{A}$ and eluted with a linear gradient $(0$ 
to $100 \%$ in 40 or 60 minutes, respectively) of buffer A containing $1 \mathrm{mmol} / \mathrm{L} \mathrm{CaCl}, 0.5$ $\mathrm{mol} / \mathrm{L} \mathrm{NaCl}$, and $0.1 \%$ isopropanol. Fractions of 0.5 to $2 \mathrm{~mL}$ were collected and assayed for trypsinogen-1 and -2 . Trypsinogen isoenzymes isolated by immunoaffinity chromatography were chromatographed in the same manner.

Immunoaffinity purified trypsinogen preparations were further purified by RP HPLC on either 8 x $100 \mathrm{~mm} \mu$-Bondapak C18 RadialPAK or $3.9 \times 75 \mathrm{~mm}$ Nova-Pak C18 column (Waters), C1 (2.1 x $100 \mathrm{~mm})$ or C4 (3.9 x 20 $\mathrm{mm}$ ) column. Trypsinogen was eluted with a linear gradient of $0.1 \%$ TFA in ACN. A HPLC system from LKB, ÄKTA prime or ÄKTA purifer (Amersham Biosciences) was used.

\section{Activation of trypsinogen iso- enzymes (I and II)}

Trypsinogen isoenzymes were purified by ion exchange HPLC and divided to three aliquots. Aprotinin was added to a concentration of 70 $\mathrm{mg} / \mathrm{L}$ to two aliquots to prevent autoactivation. One of these was activated by enteropeptidase (EC 3.4.21.9, Sigma) by incubation at $23^{\circ} \mathrm{C}$ for 16 hours. The third aliquot was activated in the absence of aprotinin and activation was confirmed by assay of enzyme activity with the synthetic $p$-nitroanilidesubstrateS-2222(Kabi) (Koivunen et al., 1989). Immunoreactivity of the trypsin-aprotinin complex was compared with that of the proenzyme (I). Alternatively, trypsinogen was autoactivated in neutral $\mathrm{pH}$ at $37^{\circ} \mathrm{C}$ for 2 hours (II).

\section{Alkylation and digestion of trypsinogens (IV)}

Purified trypsinogens were reduced with dithiotreitol and alkylated with 4-vinylpyridine (Aldrich). The alkylated proteins were desalted by RP HPLC. Trypsinogen containing fractions were pooled, dried and subjected to trypsin digestion using $5 \% \mathrm{w} / \mathrm{w}$ sequencing grade trypsin (Promega Ltd). The tryptic peptides were separated by RP HPLC, collected and analyzed by MALDI-TOF mass spectrometry.

For further digestion with chymotrypsin, selected peptides were dried and dissolved in $50 \mathrm{mmol} / \mathrm{L}$ Tris- $\mathrm{HCl}$ buffer containing $0.6 \mathrm{~mol} / \mathrm{L}$ urea. Five \% (w/w) chymotrypsin (Sigma) was added and digestion was carried out at $37^{\circ} \mathrm{C}$ overnight. The chymotryptic peptides were separated by RP HPLC and collected as above.

\section{Mass spectrometry (IV)}

Matrix-assisted laser desorption ionisation time of flight (MALDI-TOF) MS was performed with a Biflex MALDI-TOF mass spectrometer (Bruker-Daltonics) equipped with a nitrogen laser operating at $337 \mathrm{~nm}$. Before MALDI-TOF analysis, the protein digests were desalted using poros R3 (PerSeptive Biosystems) material. The peptides were eluted directly onto the MALDI sample plate with $\alpha$-cyano-4-hydroxy-cinnamic acid matrix in $0,1 \%$ TFA, $50 \%$ ACN. RP HPLC -purified proteolytic peptides were pipetted directly onto a MALDI sample plate with an equal volume of matrix and dried under a gentle stream of warm air. Peptides were analyzed either in the positive ion reflector or linear mode.

Electrospray ionization (ESI) MS analyses of purified trypsinogens and their digests were performed using a Micromass Q-TOF quadrupole/time-of-flight hybrid mass spectrometer (Q-TOF Micro, Waters). For analysis of intact proteins, the trypsinogens were injected into the mass spectrometer via a nanoflow interface with a Hamilton-syringe pump. The digested peptides were injected into the mass spectrometer after fractionation by nanoscale RP-HPLC on the CapLC (Waters) with a $0.075 \times 150 \mathrm{~mm} \mathrm{C} 18$ column (Symmetry C18, $300 \AA$, $3.5 \mu \mathrm{m}$, Waters) that was eluted with a linear gradient of ACN $(5-50 \%$ in 30 $\mathrm{min}$ ) in $0.1 \%$ formic acid. Flow rate was 0.25 $\mu \mathrm{L} / \mathrm{min}$ and the eluent was directly injected into the mass spectrometer. The capillary voltage was $2000 \mathrm{~V}$ and the source block temperature $120{ }^{\circ} \mathrm{C}$. The sampling cone was 
operated normally at $45 \mathrm{~V}$, but at in-source dissociation experiments ramped from 30 to $70 \mathrm{~V}$. Tandem mass spectrometric (MSMS) fragmentation spectra of the peptides were acquired by colliding the doubly or triply charged precursor ions with argon collision gas at accelerating voltages of $30-45 \mathrm{~V}$. As sulfotyrosine and phosphotyrosine standard peptides we used DsYMGWMDF (1134.442 Da) from Bachem and YRMKKKDEGSpYT (1584.714 Da) synthetized on a 433Aautomatic peptide synthetizer (Applied Biosystems).

\section{Data analysis (IV)}

Data analysis of intact protein ESI mass spectra was carried out with MassLynx software (Waters) and PAWS proteomic analysis software (ProteoMetrics). Mass spectra collected during the LC-MS separation of digested peptides were exported into ASCII text files using the DataBridge of the MassLynx software (Waters). The text files were imported into the DeCyder MS software (GE Healthcare), where different elution profiles were visualized as two-dimensional graphs and different $\mathrm{m} / \mathrm{z}$ values deconvoluted into molecular masses of 700-6000 Da. The ion counts of all different charge states of the same peptide were taken into account to calculate total intensity of the deconvoluted masses.

\section{N-terminal sequence analysis (IV)}

$\mathrm{NH}_{2}$-terminal sequence analyses were performed by the Edman degradation using a Procise 494A sequencer (Applied Biosystems).

\section{Electrophoresis and immuno- blotting (II and IV)}

SDS-PAGE was performed according to Laemmli (Laemmli, 1970) under nonreducing conditions on $20 \%$ polyacrylamide Phastgels using the Phastsystem (Pharmacia) and stained with silver according the manufacturer's instructions. For Western blot analysis the proteins were separated on regular $12.5 \%$ acrylamide gels and transferred to a PVDF membrane (Immobilon-P, Millipore). The membrane was incubated with anti-phosphotyrosine antibody (clone 4G10, Upstate) and MAb 14F10 specific for trypsinogen-2. Immunoreactive proteins were detected with enhanced chemiluminescense (Super Signal West Femto Maximum Sensitivity detection kit, Pierce Biotechnology).

\section{Statistical analysis (II)}

Student's unpaired $t$ test and Mann-Whitney $\mathrm{U}$ test were used to estimate the differences between the trypsinogen levels in the various ovarian cyst fluids and serum samples, respectively. 


\section{Results}

\section{Immunoassays for trypsinogens/ trypsin and concentrations in serum samples (I, III)}

The sensitivity of the TR-IFMAs for trypsinogen- 1 and -2 was 0.1 and 0.3 $\mu \mathrm{g} / \mathrm{L}$, respectively. The sensitivity could be increased up to eight-fold by increasing the sample volume to $200 \mu \mathrm{L}$. It is reported by the manufacturer that the commercial RIA for trypsin determines trypsin-like immunoreactivity, eventually trypsinogen-1, in serum or plasma. The immunoreactivity of trypsin-1 as compared to trypsinogen-1 is reported to be $60 \%$.

For the TR-IRMA for trypsinogen-1 there was no effect of proenzyme activation on the immunoreactivity as studied by activated and aprotinin-inactivated trypsin-1. However, in the TR-IRMA for trypsinogen-2

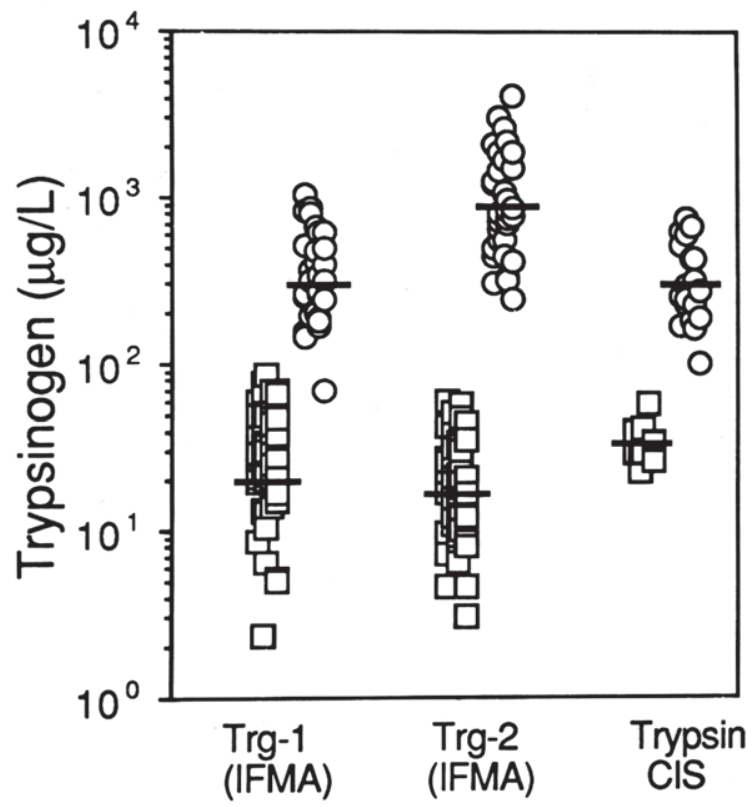

Figure 2. Serum concentrations of trypsinogen-1 (Trg-1) and -2 (Trg-2) measured by TR-IFMA and of trypsin measured by RIA form CIS in sera from healthy subjects $(\square)$ and patients with acute pancreatitis (o). Lines indicate median of each group. the immunoreactivity of the activated and aprotinin-inhibited trypsin-2 and of highly purified PMSF-inhibited trypsin- 2 was only $57 \%$ and $10 \%$, respectively. Comparison of commercial RIA for trypsin and the developed TR-IFMA for trypsinogen-1 revealed good correlation at concentrations higher than 30 $\mu \mathrm{g} / \mathrm{L}$ (Figure 2). The correlation was $\mathrm{r}=0.83$ for control samples $(n=11)$ and $r=0.90$ for pancreatitis samples $(n=20)$. Despite the reported sensitivity $(2 \mu \mathrm{g} / \mathrm{L})$ and standard curve range ( 5 to $400 \mu \mathrm{g} / \mathrm{L}$ ) RIA discriminated poorly below $30 \mu \mathrm{g} / \mathrm{L}$. TAT-2 purified from COLO 205 cell culture media by immunoaffinity and ion exchange chromatography was not detected by the RIA. For the TR-IFMAs for trypsinogen isoenzymes the cross-reaction of each isoenzyme in the assay for the other one was confirmed to be less than $1 \%$ by assaying trypsinogen-1 and -2 separated by anion exchange chromatography.

The concentration of trypsinogen-1 (median $21 \mu \mathrm{g} / \mathrm{L}$ ) was higher than that of trypsinogen-2

(median $17 \mu \mathrm{g} / \mathrm{L}$ ) in serum of healthy subjects and patients with extrapancreatic disease. The reference range of this control group for trypsinogen-1 and -2 was calculated to be 5.6 to $69 \mu \mathrm{g} / \mathrm{L}$ and 5.1 to $53 \mu \mathrm{g} / \mathrm{L}$, respectively. However, in acute pancreatitis the ratio of trypsinogen isoenzymes in serum is reversed: the concentration of trypsinogen- 2 is 50 -fold higher than in control sera, whereas the difference in trypsinogen-1 concentration is 15-fold (Figure 2). In serum samples from patients who have undergone total pancreatoduodenectomy one of nine contained trypsinogen-1 immunoreactivity $(2 \mu \mathrm{g} / \mathrm{L})$, whereas all samples contained trypsinogen-2 (median $3 \mu \mathrm{g} / \mathrm{L}$ ), the mean level being one fifth of that in control sera. The levels of trypsinogen isoenzymes, trypsinlike immunoreactivity and TATI in serum samples, ovarian follicular 


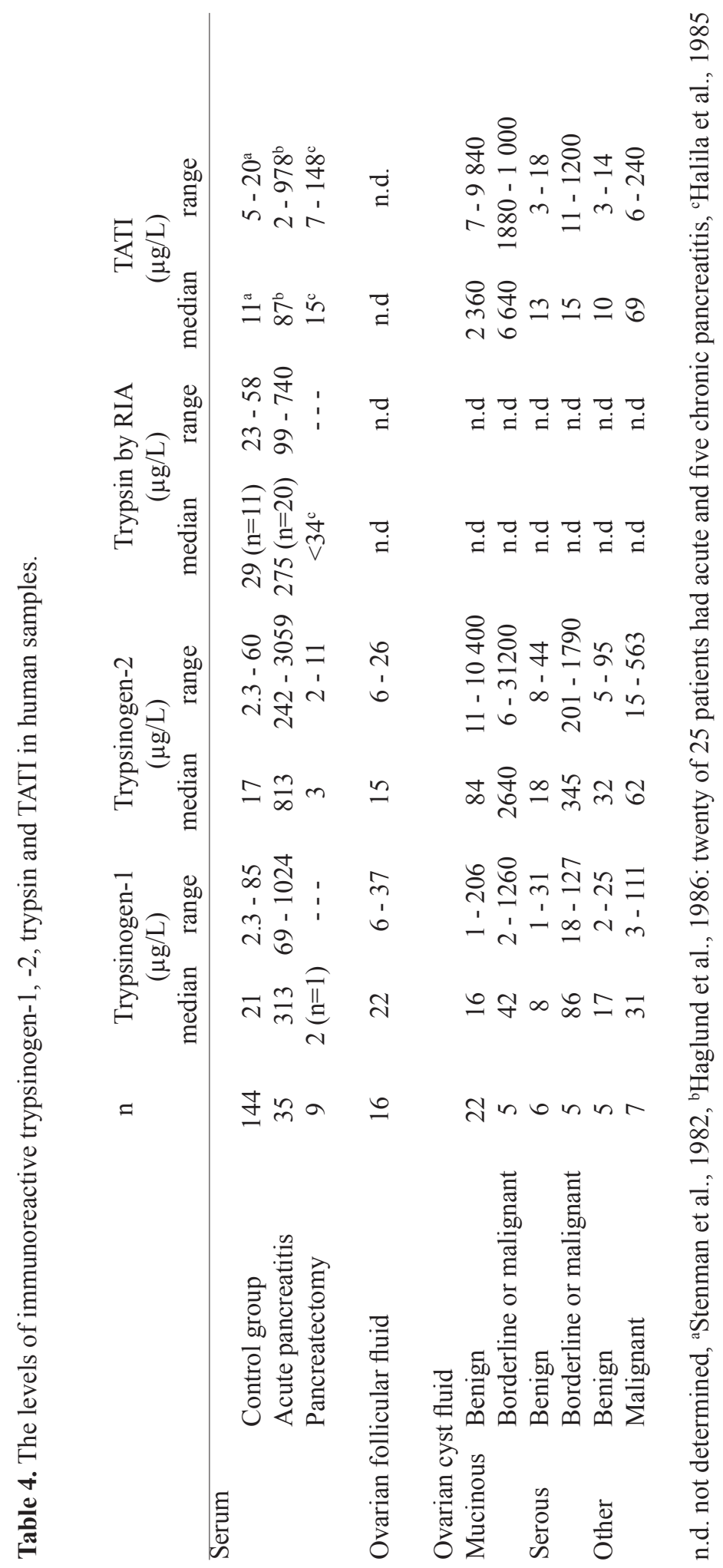


fluids and ovarian cyst fluids are summarized in Table 4.

\section{TAT and TATI concentrations in ovarian tumor cyst fluids (II)}

In hyperstimulated ovarian follicular fluid the median levels of TAT-1 $(22 \mu \mathrm{g} / \mathrm{L})$ and $-2(15$ $\mu \mathrm{g} / \mathrm{L}$ ) corresponded to those in normal serum, TAT-1 being the main isoenzyme. However, in ovarian cyst fluids TAT- 2 was the predominant form and its concentrations were significantly higher than those in control sera or ovarian follicular fluids. TAT-2 concentration was higher in mucinous than in serous cyst fluid, and especially in mucinous cyst fluids the concentration of TAT- 2 was higher in borderline or malignant (median $2640 \mu \mathrm{g} / \mathrm{L}$ ) than benign cases (median $84 \mu \mathrm{g} / \mathrm{L}$ ). Also in serous and other types of borderline and malignant ovarian carcinomas the TAT-2 concentration was higher in the benign cases (Figure 3).

Very high concentrations of TATI occurred in mucinous ovarian cyst fluids, both in benign and malignant ones (Table 4). In contrast,
TATI concentrations in serous cyst fluids (3 to $21 \mu \mathrm{g} / \mathrm{L}$ ) were similar to those in normal serum (Stenman et al., 1982) except for one malignant adenocarcinoma. TATI levels were not elevated in other benign ovarian tumors, but four malignant ones exhibited high levels (69 to $240 \mu \mathrm{g} / \mathrm{L}$ ).

\section{Characterization of trypsinogen immunoreactivity by gel filtration (I, II)}

Immunoreactive trypsinogen in serum from healthy individuals and patients with acute pancreatitis, ovarian follicle fluid and ovarian cystfluid was characterized by gel filtration. The elution pattern of these samples was identical. Trypsinogen-1 and -2 immunoreactivity eluted with molecular masses about $25 \mathrm{kDa}$ and $28 \mathrm{kDa}$, respectively, indicating that it consisted of the zymogen form, and not of trypsin. In addition, a minor peak with higher molecular mass could be seen in the assay for trypsinogen-2. This peak was later shown to represent trypsinogen-2 $-\alpha_{1}$-protease inhibitor complex (Hedström et al., 1994).

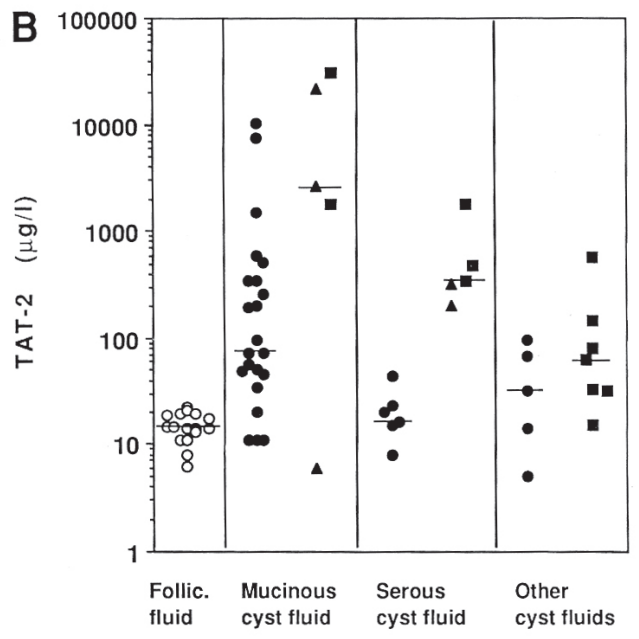

Figure 3. Concentrations of TAT-1 (A) and TAT-2 (B) in normal follicular (Follic.) fluid (O) and cyst fluid from benign $(\mathbf{O})$, borderline ( $\boldsymbol{\Delta})$, and malignant ( $\boldsymbol{\square}$ ) ovarian tumors. Bars indicate median of each group. 


\section{Trypsinogens in anion exchange chromatography (I, III)}

Trypsinogen- 1 and -2 were separated by anion exchange HPLC from serum from healthy controls, patients with acute pancreatitis and pancreatoduodenectomy, cyst fluid of mucinous ovarian cancer and conditioned culture medium from COLO 205 cells. Under identical elution conditions two immunoreactive forms of each isoenzyme

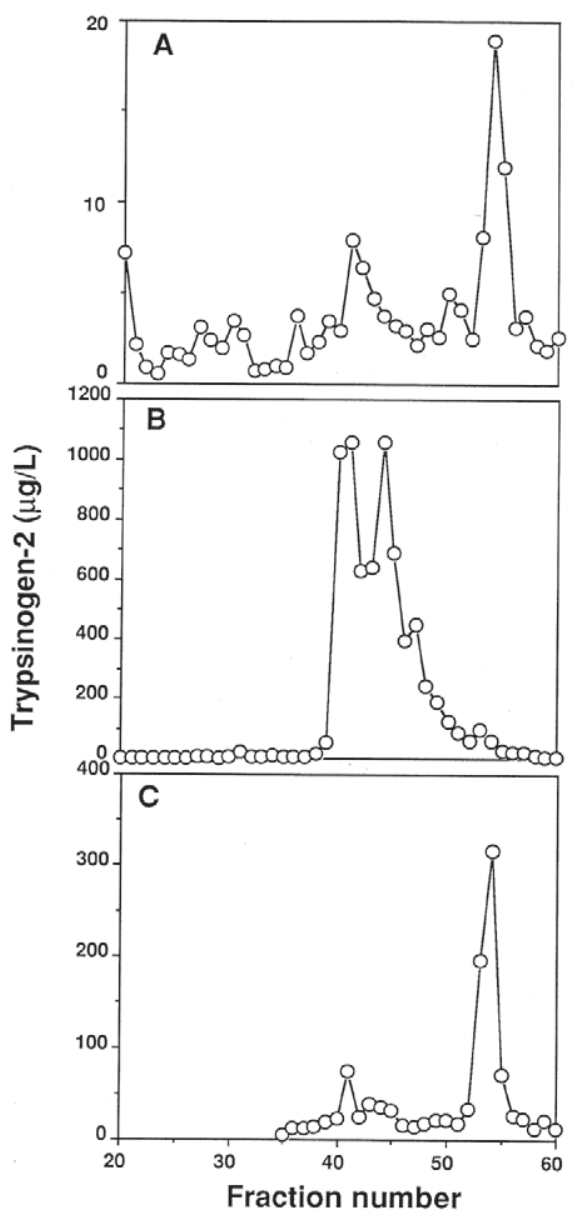

Figure 4. Comparison of elution patterns of trypsinogen-2 isoenzymes in (A) serum from a patient who had undergone pancreatectomy, (B) mucinous ovarian cyst fluid, and (C) serum from a patient with pancreatitis, as measured after anion exchange chromatography. could be seen in these samples. In the serum samples the main trypsinogen-2 peak eluted in fractions 53 to 56 (Figure 4AC) and the minor peak in fractions 40 to 43 . The less acidic, earlier eluting form of trypsinogen-2 comprised 10 to $20 \%$ of total immunoreactivity in normal serum and less than $10 \%$ of total immunoreactivity in pancreatitis serum. The latter, less acidic one corresponded to the main form of trypsinogen-2 in mucinous ovarian cyst fluid and conditioned medium of COLO 205 cells. The more acidic, main form of

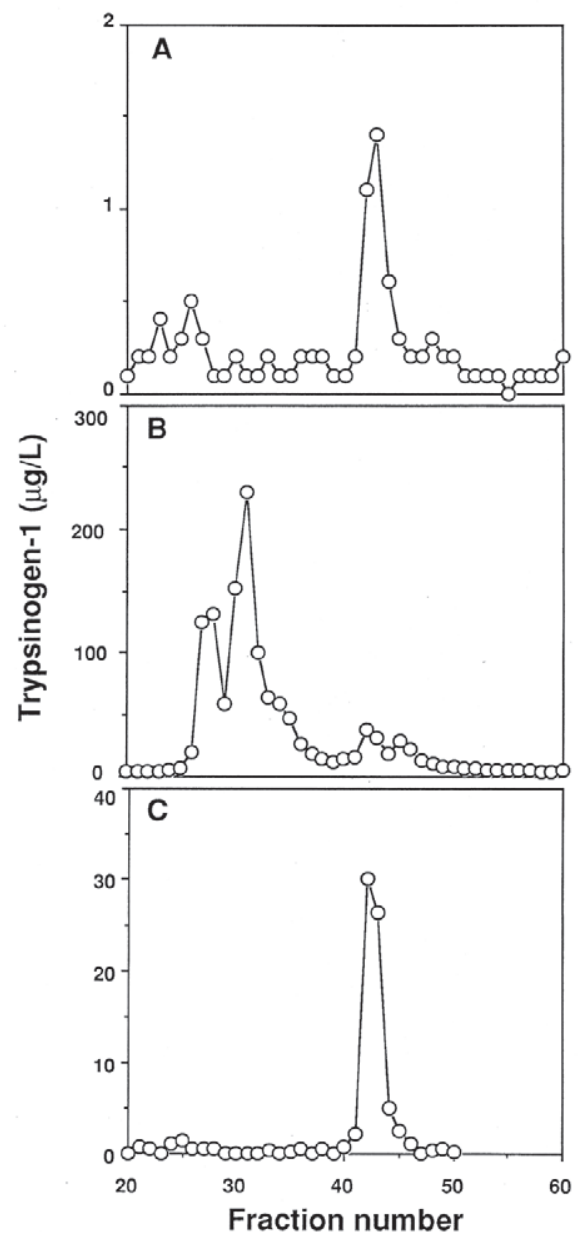

Figure 5. Comparison of elution patterns of trypsinogen-1 isoenzymes in (A) serum from a patient who had undergone pancreatectomy, (B) mucinous ovarian cyst fluid, and (C) serum from a patient with pancreatitis, as measured after anion exchange chromatography under equal conditions as in figure 4. 
trypsinogen-2 in serum could not be detected in cyst fluid of ovarian cancer (Figure 4B).

The serum samples contained one main trypsinogen-1 peak eluting in fractions 41 to 47 , and a minor, less acidic peak comprising less than $5 \%$ of the total immunoreactivity in fractions 26 to 33 (Figure 5AC). In mucinous ovarian cyst fluid and conditioned culture medium from COLO 205 cells the latter form was predominant, whereas the later eluting, more acidic trypsinogen-1 form comprised only $5 \%$ to $10 \%$ of the total immunoreactivity in ovarian cyst fluid (Figure 5B).

\section{Purification of trypsinogen by reverse-phase HPLC (II, IV)}

Immunoaffinity purified trypsinogen isoenzymes from mucinous ovarian cyst fluid, pancreatic juice and conditioned medium from COLO 205 cells were further purified by RP HPLC. To confirm the immunoreactivity measured by TR-IFMA, trypsinogen- 2 was autoactivated and purified by RP HPLC. The fractions containing trypsin activity were analyzed by SDS-PAGE and revealed a major band of $27 \mathrm{kDa}$ corresponding to trypsin-2.

\section{MS-analysis of trypsin and trypsinogen isoenzymes (IV)}

Purified trypsin and trypsinogen isoenzymes were analyzed by ESI-MS and the intact proteins gave clear $\mathrm{m} / \mathrm{z}$ envelope. The trypsins appeared in the mass spectra mainly in charge states from $[\mathrm{M}+11 \mathrm{H}]^{11+}$ to $[\mathrm{M}+16 \mathrm{H}]^{16+}$. Deconvolution of these spectra showed that the mass of trypsin-1 was 24185.0 Da, as earlier reported (Gaboriaud et al., 1996) and
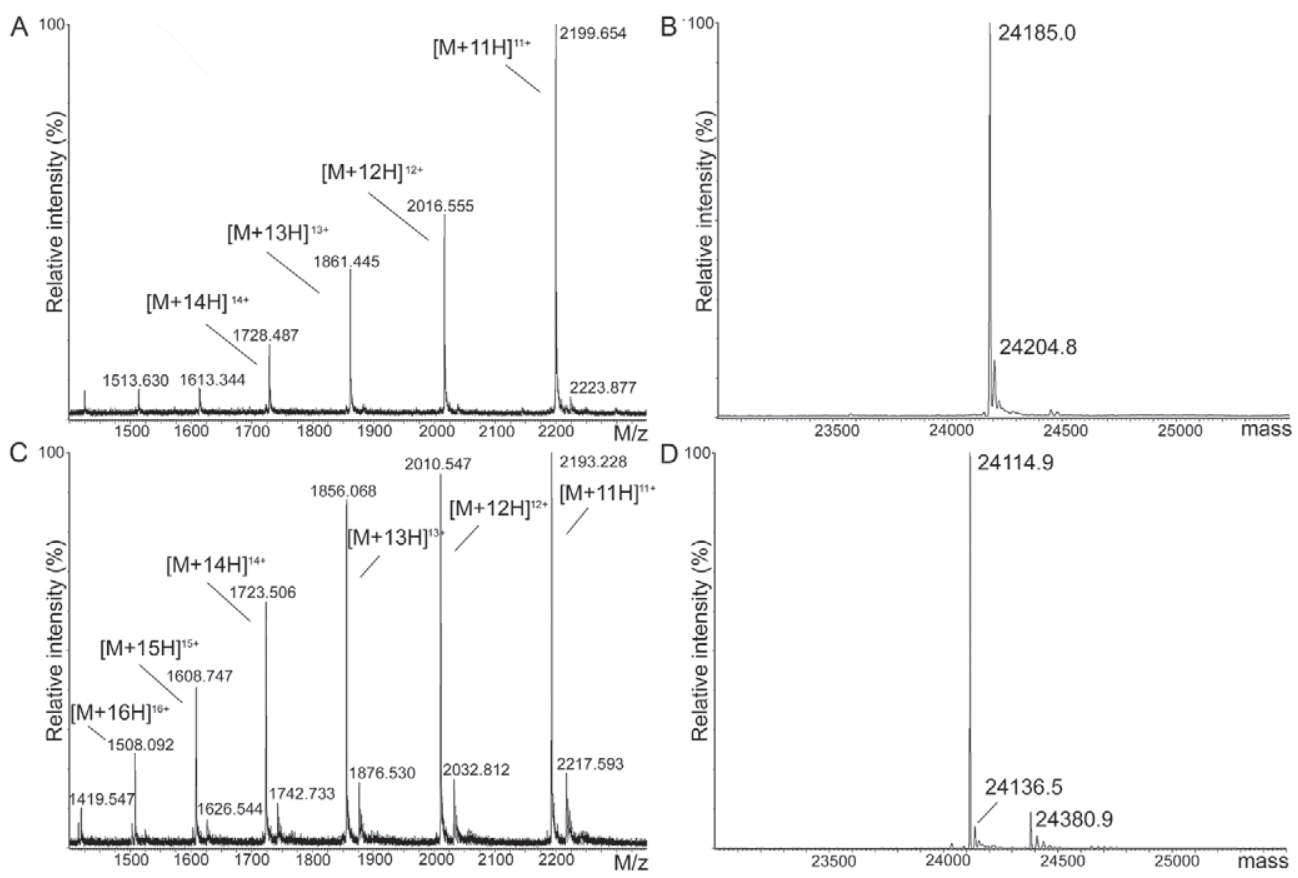

Figure 6. Mass spectrometric analyses of trypsin isoenzymes purified from pancreatic juice. ESI mass spectra of trypsin-1 (A) and -2 (C) reveal peaks mainly of $[\mathrm{M}+11 \mathrm{H}]^{11+}$ to $[\mathrm{M}+16 \mathrm{H}]^{16+}$ of the molecule as indicated. The deconvoluted spectra show that the mass of trypsin- 1 is 24185.0 (B) and that of trypsin-2 is 24114.9 (D). 
that of trypsin-2 was 24114.9 Da (Figure 6). Both of the masses are 80 Da higher than the theoretical masses calculated for trypsin-1 (24104.2 Da) and -2 (24033.9 Da) with all the putative disulfide bridges present and the propeptide cleaved as a result of activation.

Similar results and mass addition of $80 \mathrm{Da}$ were obtained with the proenzymes: the masses were 25086.0 Da and 25016.0 Da for trypsinogen-1 and -2 , respectively. However, the deconvoluted mass of TAT-2 purified from conditioned medium of COLO 205 cells was $24937.0 \mathrm{Da}$, which corresponds to the theoretical mass calculated for trypsinogen-2 (24937.8 Da) with all putative disulfide bridges and the propeptide present (Figure 7).

\section{Identification of the tryptic peptide with 80 Da mass addition (IV)}

Highly purified pancreatic trypsinogen isoenzymes were alkylated and digested with trypsin. The digests were analyzed by MALDI-TOF and LC-MS. Two-dimensional visualization of the LC-MS spectra revealed a tryptic peptide with the mass of 3598.618 as $[\mathrm{M}+4 \mathrm{H}]^{4+}$ with $\mathrm{m} / \mathrm{z}$ of 900.654 comprising trypsinogen-1 amino acids 139 to 170 with an 80 Da mass addition (Figure 8A). Similarly, a peptide with the mass off 5923.850 was observed as $[\mathrm{M}+5 \mathrm{H}]^{5+}$ with $\mathrm{m} / \mathrm{z}$ of 1185.770 corresponding trypsinogen- 2 amino acids 123 to 178 with an 80 Da mass addition (Figure 8B).

\section{Identification of Tyr154 sulfation in trypsinogen-1 and -2 (IV)}

In-source dissociation. The modification in the pancreatic trypsinogen peptides was characterized by ESI-MS using increasing cone voltages to induce in-source dissociation. The peptides comprising amino acids 139 to 170 in trypsinogen-1, 123 to 178 in trypsinogen -2 , respectively, and standard peptides containing phosphotyrosine and sulfotyrosine were analyzed under equal conditions. No loss of 80 Da was observed from the phosphotyrosine standard peptide at sampling cone voltage $30 \mathrm{~V}, 45 \mathrm{~V}$ or $70 \mathrm{~V}$. In contrast, $87 \%$ of the sulfotyrosine containing peptide appeared in a non-sulfated form at low cone voltage (30 V) and the lability of the sulfogroup increased at higher cone voltages of $45 \mathrm{~V}$ and $70 \mathrm{~V}$ (Figure 9). The tryptic peptides of pancreatic trypsinogen-1 and -2 showed similar loss of $80 \mathrm{Da}$ as the standard sulfotyrosine peptide when the cone voltage was ramped from $30 \mathrm{~V}$ to $45 \mathrm{~V}$ and $70 \mathrm{~V}$. At cone voltage $30 \mathrm{~V}$ some
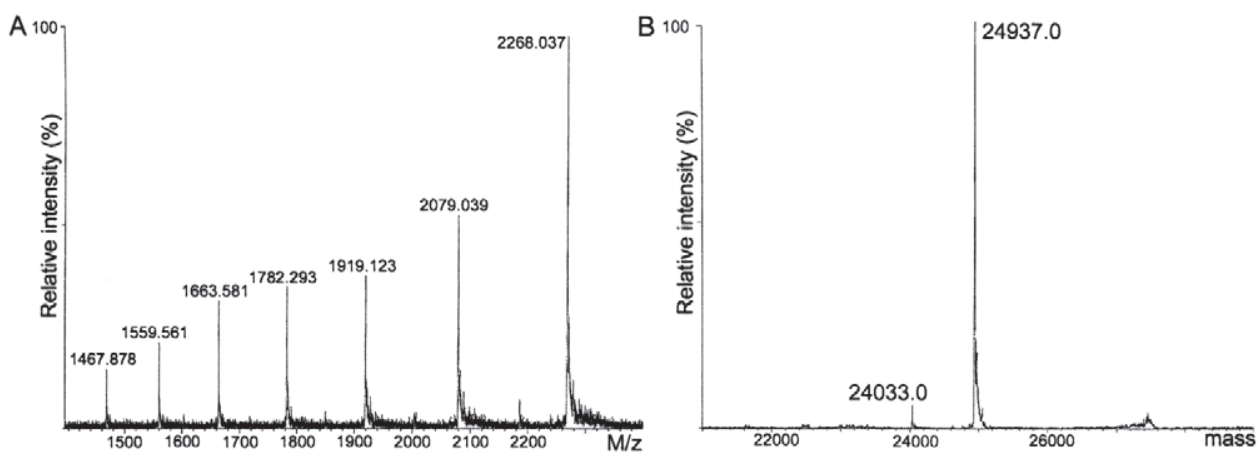

Figure 7. Mass spectrometric analysis of tumor-associated trypsinogen-2. ESI mass spectra of trypsinogen-2 purified from the medium of a colon carcinoma cell line is shown in panel A. The deconvoluted spectra indicates the mass of tumor-associated trypsinogen-2 to be 24 937.0 (B), which corresponds to the theoretical mass of the peptide without post-translational modifications. 

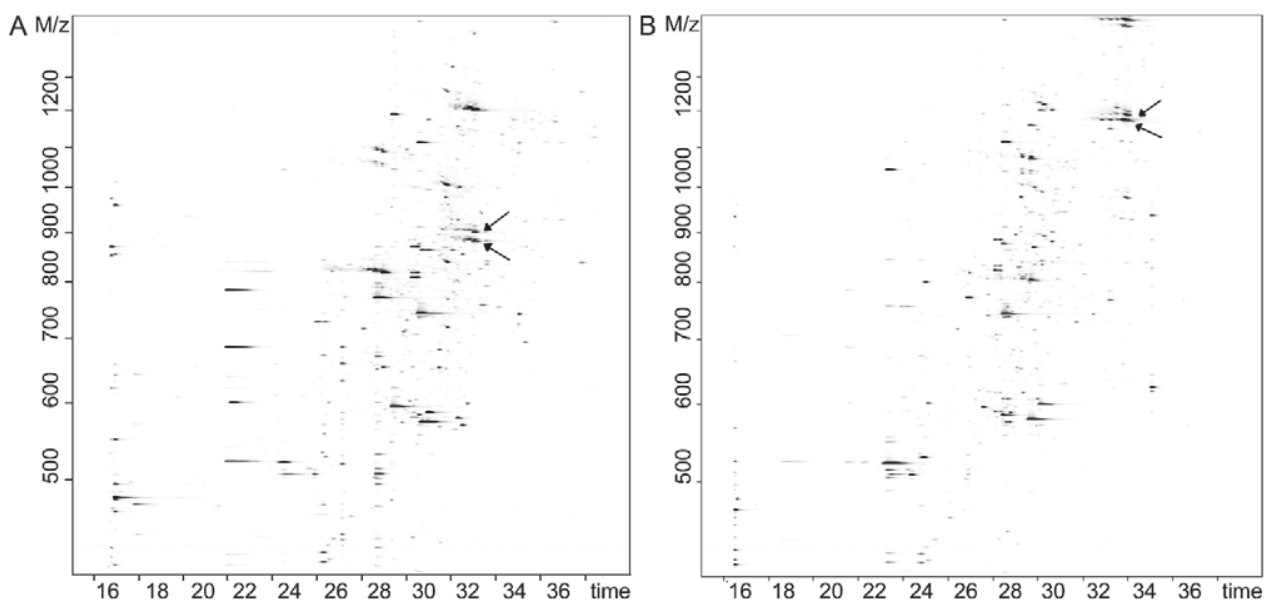

\begin{tabular}{|c|c|c|c|}
\hline Peptide & \begin{tabular}{|l} 
Position \\
(amino acids)
\end{tabular} & $\begin{array}{r}\text { Theoretical } \\
\mathrm{m}(\mathrm{Da})\end{array}$ & $\begin{array}{r}\text { Observed } \\
\mathrm{m}(\mathrm{Da}) \\
\end{array}$ \\
\hline $\mathrm{P} 1$ & $16-23$ & 921,371 & \\
\hline $\mathrm{P} 2^{\mathrm{a}}$ & $24-66$ & 5022,299 & 5022,359 \\
\hline P5 & $73-92$ & 2224,112 & 2224,135 \\
\hline P7 & 96-101 & 814,372 & \\
\hline P9 & $103-112$ & 1173,643 & 1173.647 \\
\hline $\mathrm{P} 12$ & $123-138$ & 1539,851 & 1539,871 \\
\hline $\mathrm{P} 13^{\mathrm{a}}$ & $139-170$ & 3518,648 & 3518,659 \\
\hline $\mathrm{P} 13^{\mathrm{a}}+80$ & & 3598,648 & 3598,618 \\
\hline $\mathrm{P} 14^{\mathrm{a}}$ & $171-178$ & 958,422 & 958.426 \\
\hline $\mathrm{P} 15^{\mathrm{a}}$ & $179-193$ & 1706,816 & 1706,842 \\
\hline $\mathrm{P} 16^{\mathrm{a}}$ & $194-223$ & 3265,438 & 3265.455 \\
\hline P17 & $224-231$ & 905,497 & \\
\hline P18 & 232-237 & 784,412 & 784.425 \\
\hline
\end{tabular}

\begin{tabular}{|c|c|c|c|}
\hline Peptide & \begin{tabular}{|l|}
$\begin{array}{l}\text { Position } \\
\text { (amino acids) }\end{array}$ \\
\end{tabular} & $\begin{array}{r}\text { Theoretical } \\
\mathrm{m}(\mathrm{Da}) \\
\end{array}$ & $\begin{array}{r}\text { Observed } \\
\mathrm{m} \text { (Da) } \\
\end{array}$ \\
\hline $\mathrm{P} 1$ & $16-23$ & 921,371 & \\
\hline $\mathrm{P} 2^{\mathrm{a}}$ & $24-66$ & 4994,329 & 4994,343 \\
\hline P5 & $73-92$ & 2224,112 & 2224,094 \\
\hline P9 & 103-112 & 1156,670 & 1156,665 \\
\hline P10 & $113-122$ & 1042,577 & 1042.577 \\
\hline $\mathrm{P} 11^{\mathrm{a}}$ & $123-178$ & 5843,786 & 5843,749 \\
\hline $\mathrm{P} 11^{\mathrm{a}}+80$ & & 5923,786 & 5923,850 \\
\hline $\mathrm{P} 12^{\mathrm{a}}$ & $179-193$ & 1733,827 & 1733,840 \\
\hline $\mathrm{P} 13^{\mathrm{a}}$ & $194-223$ & 3207.439 & 3207.411 \\
\hline P14 & $224-231$ & 933.503 & 933,504 \\
\hline P15 & $232-240$ & 1198,602 & 1198.609 \\
\hline
\end{tabular}

"ethylpyridyl-modified cysteines

aethylpyridyl-modified cysteines

Figure 8. Tryptic peptides of trypsinogen-1 and -2. Panels A and B show LC-MS separation of pancreatic trypsinogen- 1 and -2 tryptic peptides, respectively. Masses of tryptic peptides derived from 4-vinylpyridine-alkylated trypsinogen- 1 and -2 are shown in panels $\mathrm{C}$ and $\mathrm{D}$, respectively. The observed masses are mean values calculated on the basis of the many observed $\mathrm{m} / \mathrm{z}$ values and corresponding charge states. Peptide sequences assigned to each observed mass is indicated by amino acid positions and the theoretical mass of the assigned sequence are shown. The $[\mathrm{M}+4 \mathrm{H}]^{4+}$ of trypsinogen-1 peptide and $[\mathrm{M}+5 \mathrm{H}]^{5+}$ of trypsinogen-2 peptide containing a $80 \mathrm{Da}$ mass addition are visualized with upper arrows and the same charge states of the same peptides without the mass addition are visualized with lower arrows in panels A and B, respectively.

loss of $80 \mathrm{Da}$ could be detected and at $70 \mathrm{~V}$ more than $82 \%$ of the peptides lost $80 \mathrm{Da}$ from their mass.

Collision-induceddissociation. Thesulfation of the trypsinogen peptides was further supported by the results obtained from collision-induced dissociation (CID) in LC-MSMS. Under CID conditions no loss of $\mathrm{HPO}_{3}$ moiety from the standard phosphopeptide was observed (Figure 10 ) as earlier also reported (Nemeth-Cawley et al., 2001). Our CID analysis on mass modified trypsinogen-1 peptide (amino acids 139 to 170 ) and trypsinogen-2 peptide (amino acids 147 to 178 produced by in-source dissociation from peptide comprising amino acids 123 to 178) reveal an unmodified tyrosine at position 154 indicating loss of the $\mathrm{SO}_{3}$ moiety before 


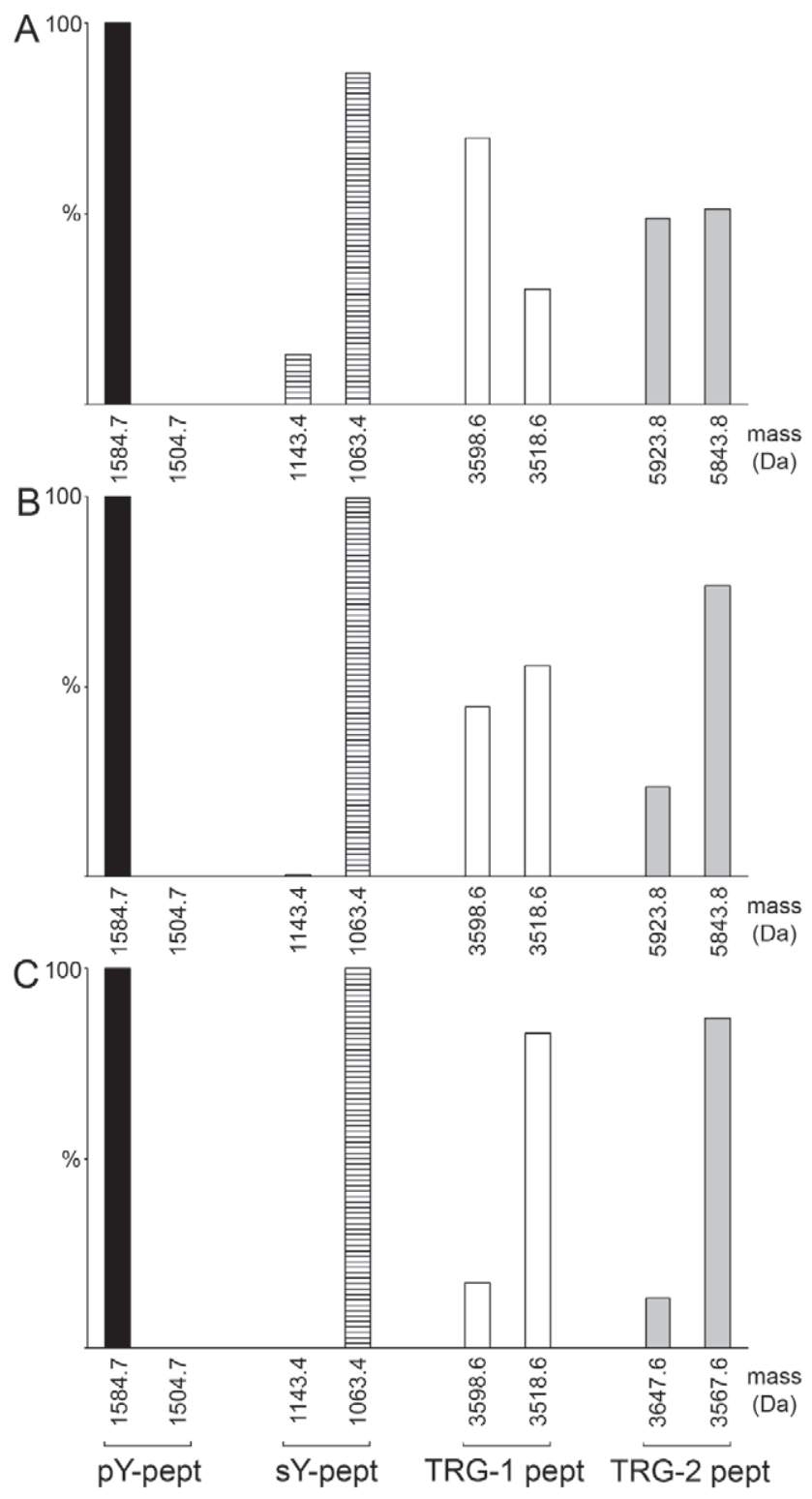

Figure 9. In-source dissociation of the sulfate group of the modified trypsinogen-1 and -2 peptides. Phosphotyrosine (black, pY-pept) and sulfotyrosine (striped, sY-pept) containing peptides together with trypsinogen-1 peptide comprising amino acids 139-170 (white, TRG-1 pept) and trypsinogen-2 peptide of amino acids 123-178 (grey, TRG-2 pept) were analyzed by LC-MS using cone voltages of $30 \mathrm{~V}(\mathrm{~A}), 45 \mathrm{~V}(\mathrm{~B})$ and $70 \mathrm{~V}(\mathrm{C})$. The proportion of sulfated peptide decreases with increasing voltage. At $70 \mathrm{~V}$ a peptide bond in the trypsinogen-2 peptide is broken resulting in a peptide consisting of amino acids 147-178, which is visualized in panel C. Different charge states of the same peptides were detected, their intensities integrated and the total intensity of the deconvoluted masses shown were calculated. The proportions of peptides with (left) and without (right) the 80 Da mass modification are indicated. 
the backbone fragmentation (Figure 10).

Immunoblotting and Edman degradation. In immunoblotting experiment with antiphosphotyrosine antibody no signal of pancreatic trypsinogen-2 was detected. Furthermore, fifteen cycles of N-terminal sequence analysis by Edman degradation of the purified chymotryptic peptide of pancreatic trypsinogen-2 comprising amino acids 149 to 178 gave a sequence SSGADYPDELQCLDA. The signal of the phenylthiohydantoin (PTH) derivative of tyrosine in position six (Tyr154) corresponds to the signals of the amino acid derivatives obtained from the other positions.
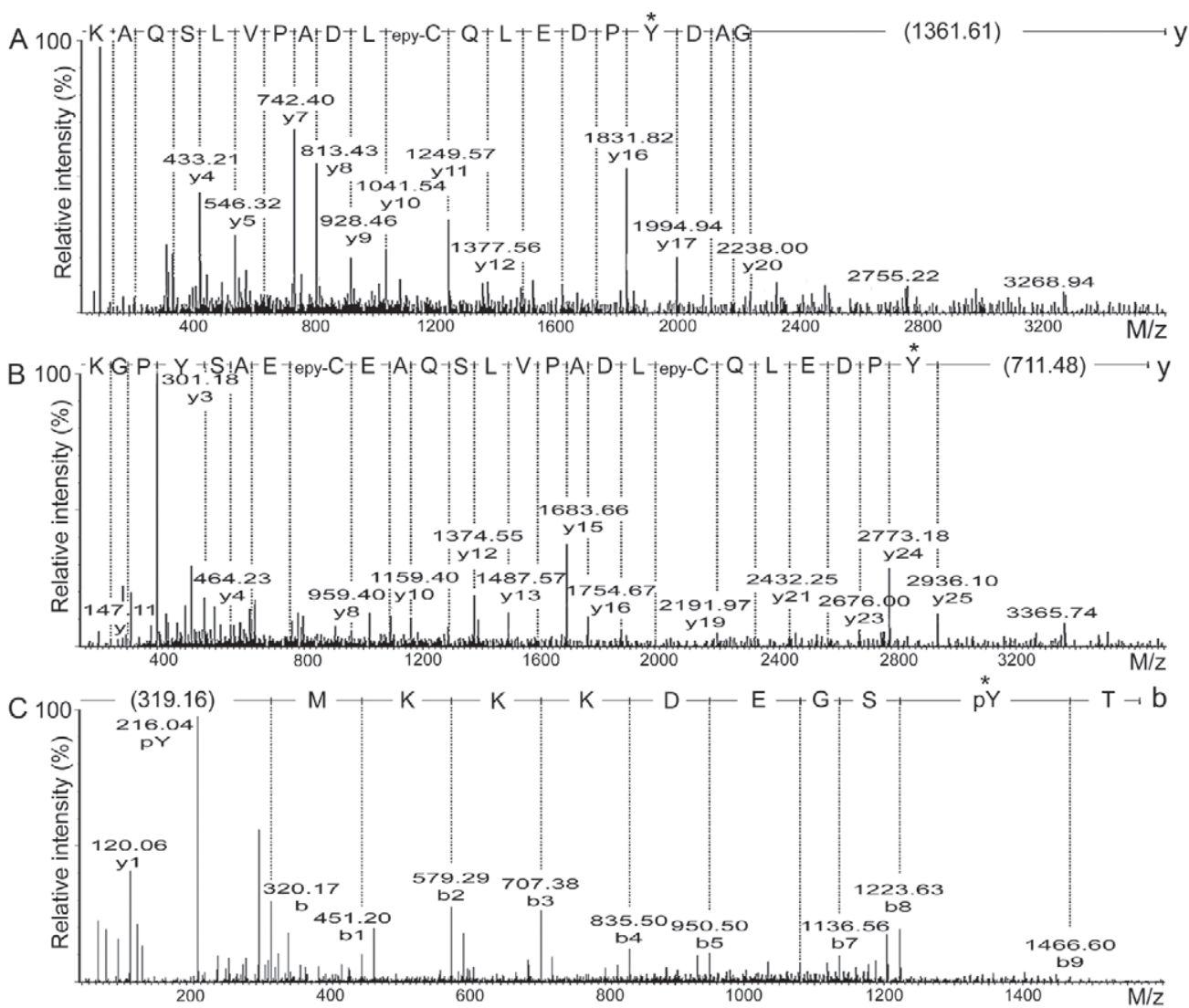

Figure 10. MSMS fragmentation spectra of tryptic peptides containing the modified amino acid (Tyr154) in pancreatic trypsinogen isoenzymes. Panel A shows the MSMS fragmentation spectra of the triply charged precursor ion of $\mathrm{m} / z 1200.54$ representing a sulfated tryptic peptide (mass 3598.60) comprising amino acids 139-170 in trypsinogen-1. The MSMS spectra of the triply charged precursor ion of $m / z 1216.55$ (mass 3646.63) is likely to represent in-source dissociated fragment of the tryptic peptide of trypsinogen-2 containing amino acids 147-178 (panel B). The peptide sequences derived from fragmented y-ion series are annotated on the spectra. Cysteine alkylated with an ethylpyridyl group is abbreviated epy-C. Panel C shows the fragmentation spectra of a phosphotyrosine-containing synthetic peptide (mass 1584.71), in which the fragmentation derived b-ion series is annotated on the spectra. The sulfated tyrosine residues of the precursor ions are indicated by asterisks. 


\section{Discussion}

\section{TR-IFMAs for trypsinogen-1 and -2}

The first aim of the present study was to produce monoclonal antibodies to trypsinogen isoenzymes identified in cyst fluid of ovarian cancer patients. We were able to produce several high affinity MAbs by immunization of mice with a preparation containing both TAT-1 and -2 isoenzymes. These MAbs were suitable for the development of immunofluorometric assays, and for purification and detection of trypsinogen isoenzymes by various immunologic techniques.

We developed highly sensitive and specific TR-IFMAs for trypsinogen-1 and -2 based on microtiterplate technology. The TR-IFMAs are sandwich-type assays with, by design, specificity for two epitopes on a molecule. The catcher MAb is immobilized onto microtiterplate wells in high concentration resulting in high sensitivity, wide analytical range and high recovery (Alfthan, 1986). The tracer MAb is labeled with lanthanide europium. Five to fifteen Eu molecules can be incorporated into an antibody molecule (Soini and Kojola, 1983) and high tracer concentration contributes to assay sensitivity (Alfthan, 1986). The lanthanide chelates have long Stokes' shift, narrow emission peaks and exceptional decay times, which allows easy and efficient background discrimination (Hemmilä and Laitala, 2005). This results in low background reading and high sensitivity in immunoassays when measuring time-resolved fluorescence (Hemmilä et al., 1984, Soini and Kojola, 1983, Soini et al., 1990). The shelf-life of lanthanide-labeled tracer is long (Alfthan, 1986). These properties of luminescent lanthanide chelates make them superior as compared to other absorptive, fluorescent or radioactive probes in immunoassays. The TRIFMAs have good precision due to technical convenience and robustness.

High specificity in the developed TR-IFMAs was achieved by combination of two MAbs with preferential reactivity for one isoenzyme. We compared our TR-IFMA for trypsinogen-1 with a commercial radioimmunoassay for trypsin and found that the assays correlated well at concentrations above $30 \mu \mathrm{g} / \mathrm{L}$, but the RIA discriminated poorly at low concentrations. The detection limits of the TR-IFMAs for trypsinogen- 1 and -2 were 0.1 and $0.3 \mu \mathrm{g} / \mathrm{L}$, respectively.

The immunoreactivity of DFP-inactivated trypsin-1 has been shown to be different from that of the proenzyme in some (Borgström and Ohlsson, 1976) but not in all (Lafont et al., 1995) immunoassays. Anyhow, trypsinogen-1 and trypsin-1 inhibited with aprotinin or PMSF reacted equally in our assay for trypsinogen-1. Thus, we could use trypsin-1 inhibited with PMSF as standard in the assay for trypsinogen-1, as purified trypsinogen-1 was not available in sufficient amounts. On the other hand, trypsin-2 inhibited with aprotinin or PMSF had clearly reduced immunoreactivity in the assay for trypsinogen-2. This could be the result of autodegradation of the highly purified and concentrated trypsin-2 preparation, as trypsin- 2 has been shown to be prone to autolysis (Mallory and Travis, 1973, Rinderknecht and Geokas, 1972). It appears to be important to use the proenzyme as a standard in the TR-IFMA for trypsinogen-2.

\section{Immunoreactive trypsinogen-1 and -2 in serum samples}

Healthy subjects. Trypsinogens are mainly produced by the exocrine pancreas and secreted at high concentrations into pancreatic fluid (Rinderknecht and Geokas, 1972). A small portion of trypsinogens and active trypsins escapes into the circulation, where trypsinogen remains free but active trypsins 
are rapidly inactivated mainly by $\alpha_{2} \mathrm{M}$ and API (Borgström and Ohlsson, 1978). Trypsinogen-1 concentrations in serum measured by us (median $21 \mu \mathrm{g} / \mathrm{L}$, range 2 to $85 \mathrm{~g} / \mathrm{L}$ ) are in line with those measured by a commercial RIA and by others using in-house RIA (mean 15 to $26 \mu \mathrm{g} / \mathrm{L}$ ) (Borgström and Ohlsson, 1976, Florholmen et al., 1984b, Geokas et al., 1979), IRMA ("most serum samples do not exceed $70 \mu \mathrm{g} / \mathrm{L} ")$ (Lafont et al., 1995), and ELISA (mean $28 \mu \mathrm{g} / \mathrm{L}$ ) (Kimland et al., 1989).

We could not make direct comparisons with other assays for trypsinogen-2 because such assays are not generally available. The median trypsinogen-2 concentration in serum measured in our study was $17 \mu \mathrm{g} / \mathrm{L}$ (range 2 to $60 \mu \mathrm{g} / \mathrm{L})$. Later, a reference range of 18 to 90 $\mu \mathrm{g} / \mathrm{L}$ for our assay was determined (Hedström et al., 1994). Similar results have been measured by an ELISA method (mean $21 \mu \mathrm{g} / \mathrm{L}$ ) (Kimland et al., 1989). However, Largman et al. (Largman et al., 1978) have determined a normal mean serum level of immunoreactive trypsinogen-2 of $4.5 \mu \mathrm{g} / \mathrm{L}$. This result was measured by RIA that used TLCK-inactivated trypsin-2 for calibration. The difference in immunoreactivity of trypsinogen-2 and inactivated trypsin-2 was marked at least in our assay and the difference in the standards used could explain the discrepancy.

We found that the ratio of trypsinogen- 1 to -2 in sera from healthy subjects and patients with extrapancreatic disease was 1.24 . This result is likely to reflect the trypsinogen-1 to -2 ratio of 2 in pancreatic fluid (Figarella et al., 1969, Guy et al., 1978, Rinderknecht et al., 1979). Similar ratio of 1.36 (Kimland et al., 1989) and 1.25 (Petersson et al., 1999) has been reported by others using ELISA.

Acutepancreatitispatients. Inacutepancreatitis we found that the serum concentration of trypsinogen-2 is 50-fold higher than in healthy controls, whereas the difference in trypsinogen- 1 concentrations was only 15 -fold and 10-fold when measured by TR-IFMA and RIA, respectively. Thus, in patients with acute pancreatitis the ratio of serum trypsinogen-1 to -2 was reversed. It was 0.38 as measured by us and 0.77 in another study (Petersson et al., 1999). This finding is compatible with previous reports of the relative concentrations of trypsinogen isoenzymes in pancreatic juice of patients with acute pancreatitis (Borulf et al., 1979, Rinderknecht et al., 1979). As discussed earlier, up-regulation of trypsinogen-2 may be a defensive mechanism, by which trypsin generation is significantly limited in potential pathological conditions (Kukor et al., 2003).

Our finding that the levels of trypsinogen-2 are increased in acute pancreatitis suggested that it could be used as a diagnostic marker for acute pancreatitis. This has been confirmed in other studies both in serum (Hedström et al., 1994, Hedström et al., 1996c, Hedström et al., 2001, Kimland et al., 1989, KylänpääBäck et al., 2002, Rinderknecht, 1996, Sainio et al., 1996) and especially in urine (Appelros et al., 2001, Hedström et al., 1996d, Jang et al., 2007, Kylänpää-Bäck et al., 2000, Sankaralingam et al., 2007). In general, elevated serum levels of trypsinogen-2 are associated with other pancreatic diseases as well, i.e. pancreatic cancer (Borgström and Andren-Sandberg, 1995, Hedström et al., 1996a), chronic pancreatitis (Borgström and Andren-Sandberg, 1995) and pancreas allograft rejection (Douzdjian et al., 1994, Lieberman et al., 1997, Marks et al., 1990, Perkal et al., 1992).

Pancreatectomized patients. The sensitive assays allowed us to measure concentrations of trypsinogen- 1 and -2 in serum samples from patients who had undergone pancreatectomy. All samples contained trypsinogen-2 (median $3 \mu \mathrm{g} / \mathrm{L})$, whereas trypsinogen-1 (2 $\mu \mathrm{g} / \mathrm{L})$ was detected in only one of nine samples. This result showed that the expression of trypsinogen, and especially that of trypsinogen-2, is not restricted to the pancreas. It was earlier shown that human Paneth cells in the intestine express trypsinogen immunoreactivity (Bohe et al., 1984), and the expression of trypsinogen isoenzymes in various normal tissues has now been confirmed by several studies (Cederqvist et al., 2003, Cottrell et al., 2004, Critchley et 
al., 2000, Ghosh et al., 2002, Kawano et al., 1997, Koshikawa et al., 1997, Koshikawa et al., 1998, Paju et al., 2000, Stenman et al., 2005, Wiegand et al., 1993).

\section{Immunoreactive trypsinogen-1 and -2 in cancer}

Several proteases have been shown to be up-regulated in cancer (Barsky et al., 1983, Diamandis et al., 2003, Hasui et al., 1989, Liotta et al., 1980, Malhotra et al., 2002, de Bruin et al., 1988). We found this to be the case also for TAT. The most interesting finding was that TAT levels, especially those of TAT-2 correlated with the degree of malignancy. We suggested that TAT could promote cellular invasion by participating in the tumor-associated protease cascade involving urokinase plasminogen activator (UPA), uPA receptor (UPAR), plasminogen and latent matrix metalloproteinases (MMPs).

Formation of metastases is the main cause of treatment failure and death for cancer patients. Remodeling of ECM in local invasion is enabled by proteinases like the MMPs and tissue serine proteinases, including tPA, uPA, plasminogen, thrombin, plasmin and trypsin (Mignatti and Rifkin, 1993, Mignatti et al., 1986). Expression of trypsinogen has been found to be associated with aggressiveness of not only ovarian tumors (Hirahara et al., 1995, Hirahara et al., 1998, Paju et al., 2001b, Paju et al., 2004, Stenman et al., 2003), but also esophageal squamous cell carcinoma (Yamamoto et al., 2001), colorectal cancer (Yamamoto et al., 2003), gastric cancer (Ichikawa et al., 2000), experimental gastric cancer in nude mice (Kato et al., 1998), prostate cancer (Bjartell et al., 2005), as well as several human cancer cell lines (Kato et al., 1998, Koshikawa et al., 1992, Miyagi et al., 1995, Miyata et al., 1998, Miyata et al., 1999). The increased expression of TAT-2 can even be measured in serum samples of patients with ovarian cancer (Paju et al., 2004), gastric cancer (Ichikawa et al., 2000), biliary and pancreatic cancer, and cholangiocarcinomas (Hedström et al., 1996a, Hedström et al., 1999,
Lempinen et al., 2007).

TAT-2 can directly activate several MMPs (Imai et al., 1995, Koivunen et al., 1989, Moilanen et al., 2003, Nyberg et al., 2002, Paju et al., 2001b, Prikk et al., 2001, Sorsa et al., 1997) and membrane bound latent matriptase (membrane type serine proteinase-1, MT-SP1) (Jin et al., 2005). MT-SP1 degrades extracellular matrix proteins and activates $\mathrm{uPa}$, hepatocyte growth factor (HGF) and PAR-2 (Lee et al., 2000) and its expression is associated with malignancy as well (Lee et al., 2005). TAT-2 has been demonstrated to efficiently degrade many ECM components (Koivunen et al., 1991a, Koshikawa et al., 1992, Moilanen et al., 2003, Stenman et al., 2005). In vitro studies have shown that cancer cell mediated degradation of ECM (Koivunen et al., 1991a) and activation of several proMMPs (Moilanen et al., 2003) is inhibited by TATI.

TAT-2 isolated from a colon carcinoma cell line is an efficient activator of PAR-2 in an in vitro study (Alm et al., 2000). The PARs are up-regulated in cancer and inflammation (Borgono and Diamandis, 2004) so TATs might also be potential in vivo activators of PAR-2. It has thus become widely accepted that TAT plays an important role in cancer progression and metastatic processes such as cellular invasion, degradation of extra-cellular matrix proteins, angiogenesis and tissue remodeling, either alone or in cascade with other proteolytic enzymes.

\section{Immunoreactive TATI in cancer}

TATI was earlier shown to be a tumor marker for mucinous ovarian tumors (Halila et al., 1988, Huhtala et al., 1982). This study shows that very high concentrations of TATI, up to $15000 \mu \mathrm{g} / \mathrm{L}$, occur in mucinous ovarian cyst fluids, both in benign and malignant ones. We also found that in some serous cyst fluids from borderline and malignant tumors the concentrations of both TAT isoenzymes were remarkably elevated but the levels of TATI were not. We thought this might reflect a disturbance in proteolytic balance, which 
may contribute to the invasive properties of malignant cells. Similar results were obtained later, when the molar ratio of trypsinogen to TATI was found to be significantly higher in serous than in mucinous cyst fluids (Paju et al., 2001b).

TAT-2 has been shown to degrade tissue inhibitor of metalloproteinase-1 (TIMP-1) (Sorsa et al., 1997), which might be one factor in disturbing the balance between proteinases and their inhibitors in cancer. The excess of proteinase in relation to its inhibitor was suggested to be related to the poorer prognosis of serous than mucinous ovarian carcinomas at an early stage of the disease by Vergote et al. (Vergote et al., 1993). High TATI expression in gastric cancer tissue seems to correlate with a favourable prognosis for the patient in one study (Wiksten et al., 2005). The finding that trypsinogen is expressed in both malignant and benign bladder epithelium, whereas TATI expression decreases with increasing stage and grade of malignancy, suggests balanced expression of trypsinogen and TATI in normal tissue, but disruption of this balance in tumor progression (Hotakainen et al., 2006). These results are suggestive of a protective role of TATI in tumour invasion, possibly by reducing the proteolytic activity of trypsin and thereby inhibiting tissue destruction and mucosal degradation.

However, in ovarian (Paju et al., 2004, Venesmaa et al., 1994, Venesmaa et al., 1998), bladder (Kelloniemi et al., 2003), prostate (Paju et al., 2007) and renal cell cancers (Paju et al., 2001a) an increased serum level of TATI is a marker of poor prognosis. In a previous report, PSTI/TATI expression correlated, in intestinal type of gastric tumours, with advanced stage tumours as well as nodal involvement (Higashiyama et al., 1990a). It has been suggested that trypsinogen and TATI are expressed simultaneously by many tumors, and an elevation of TATI in serum or urine reflects trypsinogen expression by the tumor, which in most cases is associated with aggressive disease (Paju and Stenman, 2006). Thus, the mechanisms by which trypsins and
TATI act in tumor growth and metastasis are not yet understood in detail.

\section{Characterization of pancreatic and extra-pancreatic trypsinogens}

Determination of the isoenzyme pattern by ion exchange chromatography revealed isoelectric variants of trypsinogen isoenzymes in serum samples. The less acidic forms corresponded to the main TAT-1 and -2 isoenzymes in mucinous ovarian cyst fluid, and the more acidic isoenzymes corresponded to the main peaks in serum from a patient with pancreatitis. Earlier, pancreatic trypsinogen-1 and -2 had been shown to incorporate radioactive sulfate to tyrosine residue (Scheele et al., 1981). We speculated that lack of sulfation could explain the shorter retention time of TATs in anion exchange chromatography.

Later, based on a crystal structure study and MS analysis of trypsin-1 Gaboriaud et al. (Gaboriaud et al., 1996) localized an $80 \mathrm{Da}$ modifying group at Tyr154 in the substrate binding pocket, in the S'2 subsite. As the mass of $\mathrm{HPO}_{3}$ is $79.966 \mathrm{Da}$ and that of $\mathrm{SO}_{3}$ is about $79.957 \mathrm{Da}$, the PTM was misinterpreted to be phosphate. Szilagyi et al. (Szilagyi et al., 2001) reported that two forms of trypsinogen-1 isoenzymes can be found in human pancreatic juice. Trypsin-1 with a molecular mass 24.184 Da in MS analysis, corresponding to the amino acid sequence of trypsin- 1 and an 80 Da mass addition, was obtained from pancreatic juice from one patient. This mass addition was preliminarily suggested to result from sulfation, not phosphorylation. Non-modified trypsin-1 with a molecular mass of $24.104 \mathrm{Da}$ in MS analysis was obtained from another patient's pancreatic juice. The isolation process of this trypsin contained prolonged exposure to acid environment. The authors could not explain whether the non-modified trypsin was result of hydrolytic loss of the modifying group, or was trypsinogen in fact unmodified in that sample (Szilagyi et al., 2001).

Recently, Sahin-Tóth et al. performed alkaline hydrolysis to purified human trypsinogen 
isoenzymes (Sahin-Tóth et al., 2006). Thin layer chromatography of the hydrolysates revealed existence of sulfated tyrosine residue in both trypsinogen-1 and -2 . The tyrosine sulfate residue was attributed to Tyr154 in trypsinogen-1 by incorporation of ${ }^{35} \mathrm{SO}_{4}$ to trypsinogen-1 but not to Tyr154Phe mutant trypsinogen-1 expressed in human embryonic kidney 239T cells (Sahin-Tóth et al., 2006).

In other words, we observed isoelectric variants of trypsinogen isoenzymes in serum samples by ion exchange chromatography. In the literature, there were contradictory reports of trypsinogen PTM. We therefore isolated pancreatic trypsinogen isoenzymes and TAT-2 from conditioned medium of COLO 205 cell line, and characterized intact trypsinogen isoenzymes, and tryptic and chymotryptic peptides by ESI-MS, Western blot analysis and $\mathrm{N}$-terminal sequencing.

\section{Investigation of Tyr154 modification}

Phosphorylation of trypsinogen isoenzymes (Gaboriaud et al., 1996) was excluded by immunoblotting with anti-phosphotyrosine antibody. No specific signal was detected with pancreatic trypsinogen isoenzymes, whereas the antibody efficiently recognized the Tiel tyrosine kinase, which is known to be phosphorylated on tyrosine (Saharinen et al., 2005). The presence of trypsinogen-1 and -2 isoenzymes, respectively, were verified by probing the same lanes with MAb against the respective trypsinogen isoenzyme.

A clear signal of PHT derivative of Tyr 154 was seen in Edman degradation of purified peptide from pancreatic trypsinogen-2. The fact that the PTH derivative of phosphotyrosine is hardly soluble under standard Edman sequencing conditions (Aebersold et al., 1991) indicates the absence of phosphotyrosine in position 154 of trypsinogen-2.

Mass modified trypsin peptides lost their mass addition of $80 \mathrm{Da}$ both in in-source dissociation and CID experiments, which would not occur to phosphorylated peptides. Under the CID conditions in MSMS analysis, the $\mathrm{HPO}_{3}$ moiety would remain attached to the tyrosine residue allowing site-specific identification of the phosphorylation. Tyrosine phosphorylation in peptides can be identified by detecting the immoniumion of phosphotyrosine $(\mathrm{m} / \mathrm{z} 216.04)$ in positive mode precursor ion scanning on a Q-TOF MS, and the phosphorylation site can be localized in the same experiment by MSMS fragmentation. Furthermore, potential serine or threonine phosphorylation would induce a loss of $98 \mathrm{Da}$, resulting in the appearance of dehydroalanine or dehydroamino-2-butyric acid in the MSMS fragmentation spectrum under the CID conditions used (Zhou et al., 2001). None of these was observed. In sharp contrast, it is reported (Nemeth-Cawley et al., 2001, Rappsilber et al., 2001) that loss of $\mathrm{SO}_{3}$ is the first fragmentation event in lowenergy collisionally activated dissociation, as the energy required to break the S-O bond is lower than the energy required to fragment the polypeptide backbone. Therefore, the observed loss of $80 \mathrm{Da}$ from both pancreatic trypsinogen-1 and -2 derived peptides in insource dissociation and CID experiments indicates that they are sulfated and not phosphorylated.

The pancreatic trypsinogen-1 peptide contains only one tyrosine residue, namely Tyr154, while the trypsinogen-2 peptide analyzed contains two tyrosine residues, Tyr154 and Tyr175. Tyr154 is sulfated in trypsinogen-1, and a sulfation consensus sequence surrounds Tyr154 but not Tyr175 in trypsinogen-2. Therefore, it is most probable that the Tyr 154 is also sulfated in trypsinogen-2. Taken together, our results confirm the previous findings indicating that pancreatic trypsinogen- 1 and -2 are modified at Tyr 154 and that this modification is sulfate, not phosphate (Gaboriaud et al., 1996, Sahin-Tóth et al., 2006, Scheele et al., 1981, Szilagyi et al., 2001).

On contrarily, we could show that TAT-2 from a colon carcinoma cell line is not posttranslationally modified. Instead, in MS analysis the mass of TAT- 2 corresponds 
to the theoretical mass of trypsinogen-2. This difference in sulfation at Tyr154 could explain the previously reported differences between pancreatic and tumor-associated trypsinogens (Koivunen et al., 1989). Pancreatic trypsinogens have been found to be fully sulfated suggesting extra-ordinary tyrosine sulfation capacity (Sahin-Tóth et al., 2006). TPST-1 and -2 are expressed in all tissues examined (Moore, 2003) but mRNA expression of the TPST-2 isoform is drastically higher in the pancreas than any other tissues examined (Ouyang and Moore, 1998). This might explain the incomplete sulfation of trypsinogens in extra-pancreatic tissues.

\section{Aromatic interactions in proteins}

Aromatic interactions, usually described as $\pi-\pi$ interactions, are ubiquitous in nature and are involved in many biological processes like in the antigen-binding of immunoglobulins (Padlan, 1990), stability of duplex DNA (Kool, 2001) or stabilizing protein tertiary structures (Mitchell et al., 1994, Singh and Thornton, 1990). The delocalized electrons of the benzene ring are the basis for the these interactions (Kryger et al., 1998, Kryger et al., 1999, Obst et al., 1997).

Contribution of aromatic interactions in binding affinity and ligand selectivity in the S3/ $\mathrm{S} 4$ pocket of bovine trypsin, human factor Xa and chimeric S3/S4 mutants have been studied (Di Fenza et al., 2007). The aromatic character of this pocket increases from trypsin (only Trp215) to factor Xa (Trp215, Tyr99, Phe174). The results show that the establishment of favourable directional aromatic-aromatic interactions in the $\mathrm{S} 3 / \mathrm{S} 4$ pocket with a bound ligand will increasingly contribute to binding affinity and will thus determine selectivity (Di Fenza et al., 2007). Factor Xa is thus more selective with respect to bovine trypsin for ligands which opportunely interact with the fully established aromatic box in the S3/S4 subsite.

\section{The effect of tyrosine sulfation on trypsin}

Modification of aromatic tyrosine residue by sulfation provides it with highly polarizable electrons and makes it even more electronegative. Thus, proteins and peptides become more interactive by this PTM (Lyon et al., 2000, Sasaki et al., 1999, Woods et al., 2007). Tyrosine sulfate has been shown to be involved in protein-protein interactions (Costagliola et al., 2002, Stone and Hofsteenge, 1986, Wilkins et al., 1995, Woods et al., 2007) and proteolytic activity (Michnick et al., 1994).

In trypsinogen and trypsin, Tyr154 is located in the S'2 subsite within the primary substrate binding pocket (Gaboriaud et al., 1996, Katona et al., 2002). Thus, it is likely that sulfation of Tyr154 in trypsin contributes to more efficient substrate binding. Indeed, autoactivation of sulfated trypsinogen-1 was shown to be faster than that of the nonsulfated recombinant form (Sahin-Tóth et al., 2006). (Sulfated) pancreatic trypsin-1 and -2 were shown to be more effective activators of pro-uPA than (non-sulfated) TAT-1 and -2, respectively (Koivunen et al., 1989). Furthermore, modified (sulfated) trypsin-1 was shown to be more efficiently inhibited by PSTI than the non-modified form (Sahin-Tóth et al., 2006, Szilagyi et al., 2001).

On contrary to the findings of Sahin-Tóth et al. (Sahin-Tóth et al., 2006) and Szilagyi et al. (Szilagyi et al., 2001), (non-sulfated) TATs were somewhat more efficiently inhibited by TATI and soybean trypsin inhibitor than the pancreatic trypsins, whereas pancreatic trypsin-1 was more efficiently inhibited by limabean trypsin inhibitor than TAT-1 in a study of Koivunen et al. (Koivunen et al., 1989). As described above, there is evidence supporting more efficient substrate binding for the sulfated trypsin forms as compared to the non-sulfated ones. TATI has been found to be heterogenous (Huhtala et al., 1982, Kikuchi et al., 1985) so the PSTI/TATI preparations used in these studies may not necessarily be 
comparable. It is also shown that substrate binding is not only determined by the primary substrate binding site, but several distal interactions are also involved (Hedstrom et al., 1992, Hedstrom et al., 1994b). These distal binding interactions and the possible differences in the PSTI/TATI preparations used could explain the discrepancy between these results.

The enzymatic parameters of native (sulfated) pancreatic and non-modified trypsins from pancreatic juice, ovarian cyst fluid and recombinant trypsin expressed in Escherichia coli have been determined using $p$-nitroanilide peptide substrates. The kinetic constants of pancreatic and tumor-associated trypsins were similar for one substrate (S-2222), but for two substrates (S-2444 and S-2251) the $\mathrm{k}_{\text {cat }}$ for pancreatic trypsin-2 was lower and the $\mathrm{k}_{\text {cat }} / \mathrm{K}_{\mathrm{m}}$ higher than that for TAT-2 (Koivunen et al., 1989) indicating for more efficient substrate binding by (sulfated) pancreatic trypsin- 2 . In other studies using different $p$-nitroanilide peptide substrates, the catalytic activity of native (sulfated) pancreatic trypsin-1, native non-modified pancreatic trypsin-1 and nonmodified recombinant trypsin-1, respectively, was found to be practically identical (SahinTóth et al., 2006, Szilagyi et al., 2001). The nonexistent or modest differences reported in the enzymatic parameters between (sulfated) pancreatic trypsins and (non-sulfated) tumorassociated or recombinant trypsins are likely to result from the structure of the $p$-nitroanilide peptide substrates used. The Tyr 154 residue in the S'2 subsite interacts with the leaving group side of the scissile bond, not the acyl group side. In the chromogenic substrates used there is no P'2 residue, only the acyl group side (P1 to P4) with an arginine or lysine as the $\mathrm{P} 1$ residue. Thus, the influence of S'2 site on substrate binding when using $p$-nitroanilide peptide substrates is unlikely. 


\section{Conclusions}

The most important result of this study was the development of specific MAbs and TRIFMAs to trypsinogen-1 and -2 . With these we could show that:

1) in acute pancreatitis serum trypsinogen-2 is elevated 50-fold, whereas serum trypsinogen- 1 is elevated 15 -fold, suggesting that trypsinogen-2 could be a diagnostic marker for acute pancreatitis.

2) TAT-2 is the predominant form in ovarian cyst fluids and its concentrations correlate with malignancy of these tumors. Thus, TAT is likely to be involved in ovarian tumor dissemination and breakage of tissue barriers.

3) serum samples from pancreatectomized patients contain immunoreactive trypsinogen isoenzymes. These results indicate that trypsinogen is not exclusively expressed in the pancreas and certain tumors, but that it may also be produced by normal extrapancreatic tissues.
4) two forms of trypsinogen-1 and -2 , respectively, can be found in human sera and ovarian cyst fluids.

Finally, we confirmed by ESI-MS analysis that pancreatic trypsinogen-1 and -2 are sulfated and not phosphorylated at Tyr154, whereas tumor-associated trypsinogen-2 is not. We suggest that this modification may explain the previously observed differences between pancreatic and tumor-associated trypsin. 


\section{Concluding remarks}

When this study was started PSTI/TATI had been isolated from urine of an ovarian cancer patient (Huhtala et al., 1982). Two tumorassociated trypsinogen (TAT) isoenzymes had been isolated from mucinous ovarian cyst fluid and were suggested to be the target proteinases of TATI in ovarian cancer (Koivunen et al., 1989). It had also been shown by immunohistochemistry that trypsin immunoreactivity occurs in the Paneth cells of the small intestine (Bohe et al., 1986), but the function of this Paneth cell trypsinogen was not known. The human trypsinogen genes were thought to constitute a multigene family of more than ten genes (Emi et al., 1986). It was not known whether the TATs, the Paneth cell trypsinogen, and the pancreatic trypsinogens were encoded by different genes. The production of specific MAbs and development of sensitive time-resolved immunofluorometric assays in the beginning of this study facilitated new approaches to purify and characterize human trypsinogens on one hand, and to study the expression of them in various tissues and diseases on the other hand.

We established provisional referenceranges for trypsinogen-1 and -2 with the newly developed TR-IFMAs. Furthermore, we showed that especially serum trypsinogen-2 levels are strongly elevated in acute pancreatitis. These results are in line with those reported by others (Borgström and Ohlsson, 1976, Florholmen et al., 1984b, Geokas et al., 1979, Hedström et al., 1994, Kimland et al., 1989, Lafont et al., 1995, Petersson et al., 1999). We suggested that serum trypsinogen-2 could be used as a diagnostic marker for acute pancreatitis. Indeed, clinical studies employing the MAbs and TR-IFMAs developed in this study and by others have revealed that trypsinogen- 2 in serum and especially in urine is specific and sensitive marker for the diagnosis of acute pancreatitis (Appelros et al., 2001, Hedström et al., 1994, Hedström et al., 1996c, Hedström et al., 1996d, Hedström et al., 2001, Jang et al., 2007, Kimland et al., 1989, KylänpääBäck et al., 2000, Kylänpää-Bäck et al., 2002, Rinderknecht, 1996, Sainio et al., 1996, Sankaralingam et al., 2007). These studies were follwed by the development of a rapid dipstick screening test, which is commercially available (Hedström et al., 1996b). This test is more sensitive and specific than amylase, but due to both tradition and the availability of cheap reagents compatible with automatic clinical chemistry analyzers, serum amylase - despite of its known drawbacks - has remained the most often used marker for acute pancreatitis in hospital laboratories.

Apart from our results, there are no other reports showing that trypsinogen occurs in serum of pancreatectomized patients. However, it is now known that trypsinogen is expressed in several normal tissues other than the pancreas, too. Thus, the levels of trypsinogen measured by us are likely to reflect normal extrapancreatic trypsinogen expression. Trypsinogen-2 was shown to be the main isoenzyme in serum from pancreatectomized patients. It has been shown to be expressed in several extrapancreatic cells (Cederqvist et al., 2003, Ghosh et al., 2002, Koivunen et al., 1989, Koshikawa et al., 1997, Paju et al., 2000, Prikk et al., 2001, Stenman et al., 2005).

Sulfated trypsin(ogen)-1, which is more efficiently autoactivated, more stable and is less sensitive to inhibition than trypsin(ogen)-2, is the main isoenzyme in pancreatic juice (Colomb et al., 1978, Mallory and Travis, 1973, Rinderknecht and Geokas, 1972). This ensures efficient digestion of dietary proteins and activation of other dietary enzymes. It is tempting to speculate that due to the high proteolytic potential of trypsinogen-1 its expression is limited in extrapancreatic tissues, where less proteolytic potential than in digestion is 
needed. Trypsinogen-2 (or trypsinogen-3 or -4) would thus remain the main trypsinogen isoenzyme in extrapancreatic tissues.

Our finding that TAT-2 is the predominant trypsinogen form in ovarian cyst fluids and that its concentrations correlate with malignancy led to clinical studies on trypsinogen expression in other malignancies as well. TAT-2 was found to be a new potential diagnostic marker for cholangiocarcinomas (Hedström et al., 1996a, Lempinen et al., 2007) and prognostic marker for ovarian carcinomas (Paju et al., 2004). Up-regulation of TAT-2 has also been found in other cancers (Bjartell et al., 2005, Hotakainen et al., 2006). The methods developed in this study have also been used to clarify the mechanisms underlying tumor growth and metastatic processes (Koivunen et al., 1991a, Lukkonen et al., 2000, Moilanen et al., 2003, Sorsa et al., 1997). The developed MAbs and TR-IFMAs have proved to be excellent tools in the ongoing studies associated with trypsinogen quantitation, purification, and characterization.
The recent development of mass spectrometry, software tools and especially soft ionization techniques has made mass spectrometry a valuable tool in protein chemistry. We were able to determine the chemical difference between pancreatic and tumor-associated trypsinogens by ESI-MS analysis. The absence of sulfation at Tyr154 in tumor-associated trypsinogen is likely to explain the differences between these trypsinogen forms observed earlier. It is possible to produce specific MAbs to sulfotyrosine (Hoffhines et al., 2006, Kehoe et al., 2006). If all extrapancreatic trypsinogens lack sulfate, it would be possible to develop immunometric assays specific for pancreatic trypsinogen-1 and -2 . We have become aware of many biological processes other than digestion where pancreatic or extrapancreatic trypsinogens are involved. Specific determination of pancreatic and extrapancreatic trypsinogens, respectively, is therefore of potential clinical utility. 


\section{Acknowledgements}

This study was carried out at the Department of Clinical Chemistry in the University of Helsinki and at the Hospital District of Helsinki and Uusimaa - HUSLAB during the years 1988 - 2008. I am most grateful to professor Ulf-Håkan Stenman, the head of the department and the excellent supervisor of this study, for providing outstanding working facilities at my disposal, for his guidance throughout this study, and for his endless patience, support and encouragement during all these years. I admire his vast knowledge in science and his warm attitude towards other people. It is thus a pleasure to work in his laboratory. I also wish to thank professor Lasse Viinikka, the managing director of HUSLAB, docent Martti Syrjälä, the head of the HUSLAB Department of Clinical Chemistry and Hematology, and docent Esa Hämäläinen, head of the HUSLAB unit at the Department of Obstetrics and Gynecology, for their positive attitude towards my thesis and for providing me with an excellent office.

I want to thank docent Jouko Lohi and docent Olli Saksela for careful penetration into this manuscript and their constructive criticism. Their suggestions markedly improved the content of my theses.

I wish to express my gratitude to my co-authors docent Erkki Koivunen, professor Mikko Hurme, Ph.D. Henrik Alfthan, professor Tom Schröder, docent Hannu Halila, M.Sc. Sirpa Osman, docents Jari Helin, Juhani Saarinen, Nisse Kalkkinen, Konstantin I. Ivanov, and Leena Valmu. I am grateful for having had the opportunity to work with them. Especially Erkki Koivunen and Leena Valmu have been my close associates in the field of protein chemistry. I deeply admire their vast expertise, efficiency and innovativeness. I wish to thank Henrik Alfthan for his altruistic and most valuable help in immunofluorometry, data technic challenges and layout of this book.
Warm thanks are due to my current and former colleagues and my friends Jari Leinonen, Leena Riittinen, Heli Nevanlinna, Riitta Koistinen, Paula Salmikangas, Susanna Lintula, Meerit Kämäräinen, Hannu Koistinen, Wan-Ming Zhang, Annukka Paju, and all the others with whom I have had the privilege to work with in the research laboratory. I am also greatly indebted to Liisa Airas, Anja Mäki, Maarit Leinimaa, Taina Grönholm, Anne Ahmanheimo, and Marianne Niemelä for expert technical assistance and friendship. Without their help this study could not have been accomplished.

I am indebted to all my colleagues and staff at HUSLAB. It is inspiring to work with outstanding professionals within laboratory medicine. The friendship and fruitful cooperation in the various challenges in our every-day work is greatly acknowledged.

Many thanks are directed to all my friends, with whom I have been able to share the ups and downs in life away from work. Especially I want to thank my sister-in-law Leena, Anne, Mårten, Raija, Armi, Eila, Riitta, Esa, Pia and Jorma.

I owe my deep gratitude to my parents Maija and Kalle for their help and everlasting love. They have always encouraged and supported me in my studies and hobbies, and my family in all possible ways. Warm thanks are also directed to my brother Vesa and his family, all my relatives, to my mother-in-law Liisa and her whole family.

My warmest thoughts and thankfulness are reached out to my husband Tuomo and my children Teemu, Malin and Joel for their love and constant support. Had Tuomo not taken full responsibility of our family during the hectic periods of writing and dead-lines, this study could not have been finished. 
This study was financially supported by the Academy of Finland, the Finnish Cancer Institute, the Sigrid Jusélius Foundation, the Jenny and Antti Wihuri Foundation, the Ida Montin Foundation, the Alfred Kordelin Foundation, the Finnish Social Insurance Institution, the Finska Läkaresällskapet, Sairaalakemistit ry., the University of Helsinki, and the European Union (LSHT-CT-2004503011).

Helsinki, May 2008

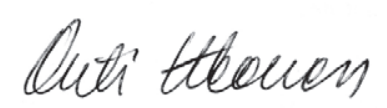

Outi Itkonen 


\section{References}

Abita JP, Delaage M and Lazdunski M. The mechanism of activation of trypsinogen. The role of the four N-terminal aspartyl residues. Eur J Biochem 8, 314-324 (1969).

Aebersold R, Watts JD, Morrison HD and Bures EJ. Determination of the site of tyrosine phosphorylation at the low picomole level by automated solid-phase sequence analysis. Anal Biochem 199, 51-60 (1991).

Aleksandrov AA, Aleksandrov LA and Riordan JR. CFTR (ABCC7) is a hydrolyzableligand-gated channel. Pflugers Arch 453, 693-702 (2007).

Alfthan H. Comparison of immunoradiometric and immunofluorometric assays for serum hCG. J Immunol Methods 88, 239-244 (1986).

Alhonen L, Parkkinen JJ, Keinänen T, Sinervirta R, Herzig KH and Jänne J. Activation of polyamine catabolism in transgenic rats induces acute pancreatitis. Proc Natl Acad Sci USA 97, 8290-8295 (2000).

Alm AK, Gagnemo-Persson R, Sorsa T and Sundelin J. Extrapancreatic trypsin-2 cleaves proteinase-activated receptor-2. Biochem Biophys Res Commun 275, 77-83 (2000).

Appelros S, Petersson U, Toh S, Johnson $\mathrm{C}$ and Borgström A. Activation peptide of carboxypeptidase $\mathrm{B}$ and anionic trypsinogen as early predictors of the severity of acute pancreatitis. Br J Surg 88 , 216-221 (2001).

Ash EL, Sudmeier JL, De Fabo EC and Bachovchin WW. A low-barrier hydrogen bond in the catalytic triad of serine proteases? Theory versus experiment. Science 278, 1128-1132 (1997).

Aubry M and Bieth J. A kinetic study of the inhibition of human and bovine trypsins and chymotrypsins by the inter-alphainhibitor from human plasma. Biochim Biophys Acta 438, 221-230 (1976).
Audrezet MP, Chen JM, Le Maréchal C, Ruszniewski P, Robaszkiewicz M, Raguenes O, Quere I, Scotet V and Feréc C. Determination of the relative contribution of three genes - the cystic fibrosis transmembrane conductance regulator gene, the cationic trypsinogen gene, and the pancreatic secretory trypsin inhibitor gene - to the etiology of idiopathic chronic pancreatitis. Eur J Hum Genet 10, 100-106 (2002).

Baeuerle PA and Huttner WB. Tyrosine sulfation is a trans-Golgi-specific protein modification. J Cell Biol 105, 2655-2664 (1987).

Baeuerle PA and Huttner WB. Tyrosine sulfation of yolk proteins 1,2 , and 3 in Drosophila melanogaster. J Biol Chem 260, 6434-6439 (1985).

Baillargeon Mary Welch Laskowski Michael, Neves Darrow E, Porubcan Michael A, Santini Robert E. and Markley, John L. Soybean trypsin inhibitor (Kunitz) and its complex with trypsin. Carbon-13 nuclear magnetic resonance studies of the reactive site arginine. Biochemistry 19, 5703-5710 (1980).

Baltimore D. Gene conversion: some implications for immunoglobulin genes. Cell 24, 592-594 (1981).

Barrett AJ, Brown MA and Sayers CA. The electrophoretically 'slow' and 'fast' forms of the alpha 2-macroglobulin molecule. Biochem J 181, 401-418 (1979).

Barsky SH, Siegal GP, Jannotta F and Liotta LA. Loss of basement membrane components by invasive tumors but not by their benign counterparts. Laboratory Investigation 49, 140-147 (1983).

Bartelt DC, Shapanka R and Greene LJ. The primary structure of the human pancreatic secretory trypsin inhibitor. Amino acid sequence of the reduced S-aminoethylated protein. Arch Biochem Biophys 179, 189-199 (1977).

Bartunik HD, Summers LJ and Bartsch HH. 
Crystal structure of bovine beta-trypsin at $1.5 \AA$ resolution in a crystal form with low molecular packing density. Active site geometry, ion pairs and solvent structure. J Mol Biol 210, 813-828 (1989).

Beatty K, Bieth J and Travis J. Kinetics of association of serine proteinases with native and oxidized alpha-1-proteinase inhibitor and alpha-1-antichymotrypsin. J Biol Chem 255, 3931-3934 (1980).

Beisswanger R, Corbeil, D, Vannier C, Thiele C, Dohrmann U, Kellner R, Ashman K, Niehrs $\mathrm{C}$ and Huttner WB. Existence of distinct tyrosylprotein sulfotransferase genes: molecular characterization of tyrosylprotein sulfotransferase-2. Proc Natl Acad Sci USA 95, 11134-11139 (1998).

Beres TM, Masui T, Swift GH, Shi L, Henke RM and MacDonald RJ. PTF1 is an organ-specific and Notch-independent basic helix-loop-helix complex containing the mammalian Suppressor of Hairless (RBP-J) or its paralogue, RBP-L. Mol Cell Biol 26, 117-130 (2006).

Bernard-Perrone F, Carrere J, Renaud W, Moriscot C, Thoreux K, Bernard P, Servin A, Balas D and Senegas-Balas F. Pancreatic trypsinogen I expression during cell growth and differentiation of two human colon carcinoma cells. Am J Physiol 274, G1077-86 (1998).

Bettelheim FR. Tyrosine-O-sulfate in a peptide from fibriongen. J Am Chem Soc 76, 2838-2839 (1954).

Bjartell A, Paju A, Zhang WM, Gadaleanu V, Hansson J, Landberg G and Stenman UH. Expression of tumor-associated trypsinogens (TAT-1 and TAT-2) in prostate cancer. Prostate 64, 29-39 (2005).

Bobofchak KM, Pineda AO, Mathews FS and Di Cera E. Energetic and structural consequences of perturbing Gly-193 in the oxyanion hole of serine proteases. J Biol Chem 280, 25644-25650 (2005).

Bode $\mathrm{W}$ and Huber R. Natural protein proteinase inhibitors and their interaction with proteinases. Eur J Biochem 204, 433-451 (1992).

Bode W, Walter J, Huber R, Wenzel HR and
Tschesche H. The refined 2.2- $\AA(0.22-\mathrm{nm})$ $\mathrm{X}$-ray crystal structure of the ternary complex formed by bovine trypsinogen, valine-valine and the Arg15 analogue of bovine pancreatic trypsin inhibitor. Eur J Biochem 144, 185-190 (1984).

Bode W, Fehlhammer H and Huber R. Crystal structure of bovine trypsinogen at 1-8 $\AA$ resolution. I. Data collection, application of patterson search techniques and preliminary structural interpretation. J Mol Biol 106, 325-335 (1976).

Bode $\mathrm{W}$ and Huber R. Induction of the bovine trypsinogen-trypsin transition by peptides sequentially similar to the N-terminus of trypsin. FEBS Lett., 68, 231-236 (1976).

Bode $\mathrm{W}$ and Schwager P. The refined crystal structure of bovine beta-trypsin at $1.8 \AA$ resolution. II. Crystallographic refinement, calcium binding site, benzamidine binding site and active site at $\mathrm{pH}$ 7.0. J Mol Biol 98, 693-717 (1975a).

Bode W and Schwager P. The single calciumbinding site of crystallin bovin betatrypsin. FEBS Lett., 56, 139-143 (1975b).

Bohe $\mathrm{H}$, Bohe $\mathrm{M}$, Lundberg E, Polling A and Ohlsson K. Production and secretion of pancreatic secretory trypsin inhibitor in normal human small intestine. $\mathrm{J}$ Gastroenterol 32, 623-627 (1997).

Bohe H, Bohe M, Jonsson P, Lindström C and Ohlsson K. Quantification of pancreatic secretory trypsin inhibitor in colonic carcinoma and normal adjacent colonic mucosa. J Clin Pathol 45, 1066-1069 (1992).

Bohe $\mathrm{M}$, Lindström $\mathrm{C}$ and Ohlsson $\mathrm{K}$. Immunoreactive pancreatic secretory trypsin inhibitor in gastrointestinal mucosa. Adv Exp.Med.Biol , 240, 101-105 (1988).

Bohe $\mathrm{M}$, Borgström A, Lindström $\mathrm{C}$ and Ohlsson K. Pancreatic endoproteases and pancreatic secretory trypsin inhibitor immunoreactivity in human Paneth cells. J Clin Pathol., 39, 786-793 (1986).

Bohe M, Borgström A, Lindström C and Ohlsson K. Trypsin-like immunoreactivity in human Paneth cells. Digestion 30, 271-275 (1984).

Böhm SK, Kong, W, Brömme D, Smeekens 
SP, Anderson DC, Connolly A, Kahn M, Nelken NA, Coughlin SR, Payan DG and Bunnett NW. Molecular cloning, expression and potential functions of the human proteinase-activated receptor-2. Biochem J 314, 1009-1016 (1996).

Borghei A, Ouyang YB, Westmuckett, A D, Marcello MR, Landel CP, Evans JP and Moore KL. Targeted disruption of tyrosylprotein sulfotransferase-2, an enzyme that catalyzes post-translational protein tyrosine $O$-sulfation, causes male infertility. J Biol Chem 281, 9423-9431 (2006).

Borgono CA and Diamandis EP. The emerging roles of human tissue kallikreins in cancer. Nat Rev Cancer 4, 876-890 (2004).

Borgström A, Appelros S, Müller CA, Uhl $\mathrm{W}$ and Büchler MW. Role of activation peptides from pancreatic proenzymes in the diagnosis and prognosis of acute pancreatitis. Surgery 131, 125-128 (2002).

Borgström A and Andren-Sandberg A. Elevated serum levels of immunoreactive anionic trypsin (but not cationic trypsin) signals pancreatic disease. Int J Pancreatol 18, 221-225 (1995).

Borgström A and Ohlsson K. Immunoreactive trypsin in serum and peritoneal fluid in acute pancreatitis. Hoppe Seylers Z Physiol Chem 359, 677-681 (1978).

Borgström A and Ohlsson K. Radioimmunological determination and characterization of cathodal trypsinlike immunoreactivity in normal human plasma. Scand J Clin Lab Invest, 36, 809-814 (1976).

Borth W. Alpha 2-macroglobulin, a multifunctional binding protein with targeting characteristics. FASEB J, 6, 3345-3353 (1992).

Borulf S, Lindberg $\mathrm{T}$. and Mansson $\mathrm{M}$. Immunoreactive anionic trypsin and anionic elastase in human milk. Acta Paediatr Scand 76, 11-15 (1987).

Borulf S, Lindberg T, Benediktsson B and Månsson M. Immunochemical determination of two trypsins in human duodenal juice. Clin Chim Acta 94, 51-62
(1979).

Bost F, Diarra-Mehrpour M and Martin JP. Inter-alpha-trypsin inhibitor proteoglycan family--a group of proteins binding and stabilizing the extracellular matrix. Eur J Biochem 252, 339-346 (1998).

Brand SJ, Andersen BN and Rehfeld JF. Complete tyrosine- $O$-sulphation of gastrin in neonatal rat pancreas. Nature 309, 456-458 (1984).

Bretaudiere JP, Tapon-Bretaudiere $\mathrm{J}$ and Stoops JK. Structure of native alpha 2-macroglobulin and its transformation to the protease bound form. Proc Natl Acad Sci USA 85, 1437-1441 (1988).

Brodrick, JW, Geokas MC, Largman C, Fassett $\mathrm{M}$ and Johnson JH. Molecular forms of immunoreactive pancreatic cationic trypsin in pancreatitis patient sera. Am J Physiol 237, E474-80 (1979).

Buck FF, Bier M and Nord FF. Some properties of human trypsin. Arch Biochem Biophys 98, 528-530 (1962).

Bundgaard JR, Vuust $\mathrm{J}$ and Rehfeld JF. Tyrosine O-sulfation promotes proteolytic processing of progastrin. EMBO J 14, 3073-3079 (1995).

Bundgaard Jens R, Vuust Jens and Rehfeld Jens F. New consensus features for tyrosine $O$-sulfation determined by mutational analysis. J Biol Chem 272, 21700-21705 (1997).

Carrell RW. Alpha 1-Antitrypsin: molecular pathology, leukocytes, and tissue damage. J Clin Invest 78, 1427-1431 (1986).

Castellani C, Bonizzato A and Mastella G. CFTR mutations and IVS8-5T variant in newborns with hypertrypsinaemia and normal sweat test. J Med Genet 34, 297-301 (1997).

Cederqvist, K, Haglund C, Heikkilä P, Sorsa T, Tervahartiala T, Stenman UH. and Andersson S. Pulmonary trypsin-2 in the development of bronchopulmonary dysplasia in preterm infants. Pediatrics 112, 570-577 (2003).

Chambers AF and Matrisian LM. Changing views of the role of matrix metalloproteinases in metastasis. J Natl Cancer Inst 89, 1260-1270 (1997). 
Chari ST. Chronic pancreatitis: classification, relationship to acute pancreatitis, and early diagnosis. J Gastroenterol, 42 Suppl 17, 58-59 (2007).

Chen JM, Cooper DN, Chuzhanova N, Feréc $\mathrm{C}$ and Patrinos GP. Gene conversion: mechanisms, evolution and human disease. Nat Rev Genet 8, $762-775$ (2007).

Chen JM, Kukor Z, Le Maréchal C, Tóth M, Tsakiris L, Raguenes O, Feréc C and Sahin-Tóth M. Evolution of trypsinogen activation peptides. Mol Biol Evol 20, 1767-1777 (2003a).

Chen JM, Le Maréchal C, Lucas D, Raguénès $\mathrm{O}$ and Feréc C. "Loss of function" mutations in the cationic trypsinogen gene (PRSS1) may act as a protective factor against pancreatitis. Mol Genet Metab 79, 67-70 (2003b).

Chen JM, Montier T and Feréc C. Molecular pathology and evolutionary and physiological implications of pancreatitisassociated cationic trypsinogen mutations. Hum Genet 109, 245-252 (2001).

Chen JM and Feréc C. Gene conversionlike missense mutations in the human cationic trypsinogen gene and insights into the molecular evolution of the human trypsinogen family. Mol Genet Metab 71, 463-469 (2000a).

Chen JM and Feréc C. Origin and implication of the hereditary pancreatitis-associated N21I mutation in the cationic trypsinogen gene. Hum Genet 106, 125-126 (2000b).

Chen JM and Feréc C. Genes, cloned cDNAs, and proteins of human trypsinogens and pancreatitis-associated cationic trypsinogen mutations. Pancreas 21, 57-62 (2000c).

Chen JM, Mercier B, Audrezet MP and Feréc C. Mutational analysis of the human pancreatic secretory trypsin inhibitor (PSTI) gene in hereditary and sporadic chronic pancreatitis. J Med Genet 37, 67-69 (2000).

Choe $\mathrm{H}$ and Farzan M. Tyrosine sulfate trapped by amber. Nat Biotechnol 24, 1361-1362 (2006).

Choe H, Moore MJ, Owens CM, Wright PL, Vasilieva N, Li W, Singh, AP, Shakri R,
Chitnis CE and Farzan M. Sulphated tyrosines mediate association of chemokines and Plasmodium vivax Duffy binding protein with the Duffy antigen/ receptor for chemokines (DARC). Mol Microbiol 55, 1413-1422 (2005).

Cockell M, Stevenson BJ, Strubin M, Hagenbuchle $\mathrm{O}$ and Wellauer PK. Identification of a cell-specific DNAbinding activity that interacts with a transcriptional activator of genes expressed in the acinar pancreas. Mol Cell Biol 9, 2464-2476 (1989).

Cocks TM, Fong B, Chow, JM, Anderson GP, Frauman AG, Goldie RG, Henry PJ, Carr MJ, Hamilton JR and Moffatt JD. A protective role for protease-activated receptors in the airways. Nature 398, 156-160 (1999a).

Cocks TM, Sozzi V, Moffatt JD and Selemidis S. Protease-activated receptors mediate apamin-sensitive relaxation of mouse and guinea pig gastrointestinal smooth muscle. Gastroenterology 116, 586-592 (1999b).

Cohn JA, Friedman KJ, Noone PG, Knowles MR, Silverman LM and Jowell PS. Relation between mutations of the cystic fibrosis gene and idiopathic pancreatitis. N Engl J Med 339, 653-658 (1998).

Collier S, Tassabehji M, Sinnott P and Strachan T. A de novo pathological point mutation at the 21-hydroxylase locus: implications for gene conversion in the human genome. Nat Genet 3, 260-265 (1993).

Colomb E and Figarella C. Comparative studies on the mechanism of activation of the two human trypsinogens. Biochim Biophys Acta 571, 343-351 (1979).

Colomb E, Guy O, Deprez P, Michel R and Figarella $\mathrm{C}$. The two human trypsinogens: catalytic properties of the corresponding trypsins. Biochim Biophys Acta 525, 186-193 (1978).

Costagliola S, Panneels V, Bonomi M, Koch J, Many MC, Smits G and Vassart G. Tyrosine sulfation is required for agonist recognition by glycoprotein hormone receptors. EMBO J 21, 504-513 (2002).

Cottrell GS, Amadesi S, Grady EF and Bunnett NW. Trypsin IV, a novel agonist 
of protease-activated receptors 2 and 4 . J Biol Chem 279, 13532-13539 (2004).

Cottrell GS, Amadesi S, Schmidlin F and Bunnett N. Protease-activated receptor 2: activation, signalling and function. Biochem Soc Trans 31, 1191-1197 (2003).

Coughlin SR. Protease-activated receptors in hemostasis, thrombosis and vascular biology. J Thromb Haemost 3, 1800-1814 (2005).

Craik CS, Roczniak S, Sprang S, Fletterick $\mathrm{R}$ and Rutter W. Redesigning trypsin via genetic engineering. J Cell Biochem 33, 199-211 (1987).

Craik CS, Largman C, Fletcher T, Roczniak S, Barr PJ, Fletterick R and Rutter WJ. Redesigning trypsin: alteration of substrate specificity. Science 228, 291-297 (1985).

Craven R.J, Lightfoot $\mathrm{H}$ and Cance WG. A decade of tyrosine kinases: from gene discovery to therapeutics. Surg Oncol 12, 39-49 (2003).

Creighton TE. In Proteins. Structures and molecular properties. WH. Freeman and company, New York, USA, 1984.

Critchley G, Sumar N, O’Neill K, HermonTaylor J and Bell B. A Cerebral trypsinogen expression in human and rat cerebrospinal fluid. Neurosci.Lett, 283, 13-16 (2000).

Crozier SJ, Sans MD, Guo L, D’Alecy LG and Williams JA. Activation of the mTOR signalling pathway is required for pancreatic growth in protease-inhibitorfed mice. J Physiol (Lond) 573, 775-786 (2006).

D'Andrea MR, Derian CK, Santulli RJ and Andrade-Gordon P. Differential expression of protease-activated receptors- 1 and -2 in stromal fibroblasts of normal, benign, and malignant human tissues. Am J Pathol 158, 2031-2041 (2001).

D'Andrea MR, Rogahn CJ and AndradeGordon P. Localization of proteaseactivated receptors- 1 and -2 in human mast cells: indications for an amplified mast cell degranulation cascade. Biotech Histochem 75, 85-90 (2000).

D'Andrea MR, Derian CK, Leturcq D, Baker SM, Brunmark A, Ling P, Darrow AL,
Santulli RJ, Brass LF and Andrade-Gordon P. Characterization of protease-activated receptor-2 immunoreactivity in normal human tissues. J Histochem. Cytochem 46, 157-164 (1998).

Darmoul D, Gratio V, Devaud H and Laburthe M. Protease-activated receptor 2 in colon cancer: trypsin-induced MAPK phosphorylation and cell proliferation are mediated by epidermal growth factor receptor transactivation. J Biol Chem 279, 20927-20934 (2004).

Darmoul D, Marie JC, Devaud H, Gratio $\mathrm{V}$ and Laburthe $\mathrm{M}$. Initiation of human colon cancer cell proliferation by trypsin acting at protease-activated receptor-2. $\mathrm{Br}$ J Cancer 85, 772-779 (2001).

de Bruin PA, Griffioen G, Verspaget HW, Verheijen JH, Dooijewaard G, van den Ingh HF. and Lamers CB. Plasminogen activator profiles in neoplastic tissues of the human colon. Cancer Res, 48, 4520-4524 (1988).

DeLeonDD, FarzadC, Crutchlow MF, Brestelli J, Tobias J, Kaestner KH and Stoffers DA. Identification of transcriptional targets during pancreatic growth after partial pancreatectomy and exendin-4 treatment. Physiol Genomics 24, 133-143 (2006).

Dery O, Corvera CU, Steinhoff $\mathrm{M}$ and Bunnett NW. Proteinase-activated receptors: novel mechanisms of signaling by serine proteases. Am J Physiol 274, 1429-1452 (1998).

Di Fenza A, Heine A, Koert U and Klebe G. Understanding binding selectivity toward trypsin and Factor Xa: the role of aromatic interactions. ChemMedChem 2, 297-308 (2007).

Diamandis EP, Scorilas A, Fracchioli S, Van Gramberen M, De Bruijn H, Henrik A, Soosaipillai A, Grass L, Yousef GM, Stenman UH, Massobrio M, Van Der Zee AG, Vergote I and Katsaros D. Human kallikrein 6 (hK6): a new potential serum biomarker for diagnosis and prognosis of ovarian carcinoma. J Clin Oncol 21, 1035-1043 (2003).

Diederichs S, Bulk, E, Steffen B, Ji P, Tickenbrock L, Lang K, Zanker KS, 
Metzger R, Schneider PM, Gerke V, Thomas M, Berdel WE, Serve $H$ and Muller-Tidow C. S100 family members and trypsinogens are predictors of distant metastasis and survival in early-stage nonsmall cell lung cancer. Cancer Res 64, 5564-5569 (2004).

Diggle CP, Cruickshank, S, Olsburgh, JD, Pellegrin S, Smith B, Banks RE, Selby PJ, Knowles MA, Southgate J and Harnden P. Identification of genes up-regulated in urothelial tumors: the 67-kd laminin receptor and tumor-associated trypsin inhibitor. Am J Pathol 163, 493-504 (2003).

Dodgson KS, Powell GM, Rose FA and Tudball N. Observations on the metabolism of tyrosine $O\left[{ }^{35} \mathrm{~S}\right]$-sulphate in the rat. Biochem J 79, 209-213 (1961).

Dodgson KS, Rose FA and Tudball N. Studies on sulphatases. 23. The enzymic desulphation of tyrosine $O$-sulphate. Biochem J 71, 10-15 (1959).

Dorfman T, Moore MJ, Guth AC, Choe H and Farzan M. A tyrosine-sulfated peptide derived from the heavy-chain CDR3 region of an HIV-1-neutralizing antibody binds gp120 and inhibits HIV-1 infection. J Biol Chem 281, 28529-28535 (2006).

Douzdjian V, Cooper JL, Abecassis MM and Corry RJ. Markers for pancreatic allograft rejection: comparison of serum anodal trypsinogen, serum amylase, serum creatinine and urinary amylase. Clin Transplant, 8, 79-82 (1994).

Duan RD, Zheng CF, Guan KL. and Williams JA. Activation of MAP kinase kinase (MEK) and Ras by cholecystokinin in rat pancreatic acini. Am J Physiol 268, G1060-5 (1995).

Dubick MA, Mar G, Mayer AD, Majumdar AP, McMahon MJ and Geokas MC. Digestive enzymes and protease inhibitors in plasma from patients with acute pancreatitis. Pancreas 2, 187-194 (1987).

Ducroc R, Bontemps C, Marazova K, Devaud $\mathrm{H}$, Darmoul D and Laburthe M. Trypsin is produced by and activates proteaseactivated receptor-2 in human cancer colon cells: evidence for new autocrine loop. Life Sci 70, 1359-1367 (2002).

Durie PR, Forstner GG, Gaskin KJ, Weizman Z, Kopelman HR, Ellis L and Largman C. Elevated serum immunoreactive pancreatic cationic trypsinogen in acute malnutrition: evidence of pancreatic damage. J Pediatr 106, 233-238 (1985).

Eddeland A and Ohlsson K. Studies on the pancreatic secretory trypsin inhibitor in plasma and its complex with trypsin in vivo and in vitro. Scand J Clin.Lab Invest, 38, 507-515 (1978).

Elias E, Redshaw M and Wood T. Diagnostic importance of changes in circulating concentrations of immunoreactive trypsin. Lancet, 2, 66-68 (1977).

Emi M, Nakamura Y, Ogawa M, Yamamoto $\mathrm{T}$, Nishide T, Mori T and Matsubara $\mathrm{K}$. Cloning, characterization and nucleotide sequences of two cDNAs encoding human pancreatic trypsinogens. Gene 41, 305-310 (1986).

Evnin LB, Vasquez JR and Craik, CS. Substrate specificity of trypsin investigated by using a genetic selection. Proc Natl Acad Sci USA 87, 6659-6663 (1990).

Farzan M, Chung S, Li W, Vasilieva, N, Wright, PL, Schnitzler CE, Marchione RJ, Gerard C, Gerard NP, Sodroski J and Choe H. Tyrosine-sulfated peptides functionally reconstitute a CCR5 variant lacking a critical amino-terminal region. J Biol Chem 277, 40397-40402 (2002).

Fehlhammer H, Bode W and Huber R. Crystal structure of bovine trypsinogen at 1-8 $\AA$ resolution. II. Crystallographic refinement, refined crystal structure and comparison with bovine trypsin. J Mol Biol 111, 415-438 (1977).

Feldman SR, Rosenberg MR, Ney KA, Michalopoulos G and Pizzo SV. Binding of alpha 2-macroglobulin to hepatocytes: mechanism of in vivo clearance. Biochem Biophys Res Commun 128, 795-802 (1985).

Feréc, C, Raguenes O, Salomon R, Roche C, Bernard JP, Guillot, M, Quere I, Faure C, Mercier B, Audrezet, M P, Guillausseau PJ, Dupont, C, Munnich A, Bignon JD and Le Bodic L. Mutations in the cationic 
trypsinogen gene and evidence for genetic heterogeneity in hereditary pancreatitis. $\mathrm{J}$ Med.Genet, 36, 228-232 (1999).

Figarella C, Miszczuk-Jamska B and Barrett AJ. Possible lysosomal activation of pancreatic zymogens. Activation of both human trypsinogens by cathepsin B and spontaneous acid. Activation of human trypsinogen 1. Biol Chem.Hoppe Seyler 369 Suppl, 293-298 (1988).

Figarella C, Negri GA and Guy O. The two human trypsinogens. Inhibition spectra of the two human trypsins derived from their purified zymogens. Eur J Biochem 53, 457-463 (1975).

Figarella C, Clemente F and Guy O. On zymogens of human pancreatic juice. FEBS Lett 3, 351-353 (1969).

Finer-Moore JS, Kossiakoff AA, Hurley JH, Earnest T and Stroud RM. Solvent structure in crystals of trypsin determined by X-ray and neutron diffraction. Proteins 12, 203-222 (1992).

Florholmen J, Burhol PG and Jorde R. Postprandial response and diurnal variation of serum cationic trypsinlike immunoreactivity in man. Scand J Gastroenterol 19, 622-625 (1984a).

Florholmen J, JordeR, Olsen RL, Kolmannskog $\mathrm{S}$ and Burhol PG. Radioimmunoassay of cationic trypsin-like immunoreactivity in man. Scand J Gastroenterol 19, 613-621 (1984b).

Fodor K, Harmat V, Neutze R, Szilagyi L, Graf L and Katona G. Enzyme:substrate hydrogen bond shortening during the acylation phase of serine protease catalysis. Biochemistry 45, 2114-2121 (2006).

Fodor K, Harmat V, Hetenyi C, Kardos J, Antal J, Perczel A, Patthy A, Katona G and Graf L. Extended intermolecular interactions in a serine protease-canonical inhibitor complex account for strong and highly specific inhibition. J Mol Biol 350, 156-169 (2005).

Fong AM, Alam SM, Imai T, Haribabu B and Patel DD. CX3CR1 tyrosine sulfation enhances fractalkine-induced cell adhesion. J Biol Chem 277, 19418-19423 (2002).
Fox MT, Harriott P, Walker B and Stone SR. Identification of potential activators of proteinase-activated receptor-2. FEBS Lett, 417, 267-269 (1997).

Freeman TC, Playford RJ, Quinn C, Beardshall $\mathrm{K}$, Poulter L, Young $\mathrm{J}$ and Calam J. Pancreatic secretory trypsin inhibitor in gastrointestinal mucosa and gastric juice. Gut 31, 1318-1323 (1990).

Frey PA, Whitt SA and Tobin JB. A low-barrier hydrogen bond in the catalytic triad of serine proteases. Science 264, 1927-1930 (1994).

Friederich E, Fritz HJ and Huttner WB. Inhibition of tyrosine sulfation in the trans-Golgi retards the transport of a constitutively secreted protein to the cell surface. J Cell Biol 107, 1655-1667 (1988).

Fukayama, M, Hayashi Y, Koike M, Ogawa $\mathrm{M}$ and Kosaki G. Immunohistochemical localization of pancreatic secretory trypsin inhibitor in fetal and adult pancreatic and extrapancreatic tissues. J Histochem. Cytochem, 34, 227-235 (1986).

Gaboriaud C, Serre L, Guy-Crotte O, Forest, E and Fontecilla-Camps JC. Crystal structure of human trypsin 1: unexpected phosphorylation of Tyr151. J Mol Biol 259, 995-1010 (1996).

Gaiser S, Ahler A, Gundling F, Kruse ML, Savkovic V, Selig L, Teich N, Tomasini R, Dagorn JC, Mossner J, Keim V and Bodeker H. Expression of mutated cationic trypsinogen reduces cellular viability in AR4-2J cells. Biochem Biophys Res Commun 334, 721-728 (2005).

Galindo J, Jones N, Powell GL, Hollingsworth SJ and Shankley N. Advanced qRTPCR technology allows detection of the cholecystokinin 1 receptor (CCK1R) expression in human pancreas. Pancreas 31, 325-331 (2005).

Gao JC, Hyeryun Bota D, Wright PL, Gerard $\mathrm{C}$ and Gerard NP. Sulfation of tyrosine 174 in the human C3a receptor is essential for binding of C3a anaphylatoxin. J Biol Chem 278, 37902-37908 (2003).

Geokas MC, Largman C, Brodrick JW and Johnson JH. Determination of human 
pancreatic cationic trypsinogen in serum by radioimmunoassay. Am J Physiol 236, E77-83 (1979).

Gettins PGW. Serpin Structure, Mechanism, and Function. Chem Rev 102, 4751-4804 (2002).

Ghosh D, Porter E Shen B, Lee SK, Wilk D, Drazba J, Yadav S, Crabb JW, Ganz T and Bevins CL. Paneth cell trypsin is the processing enzyme for human defensin-5. Nat Immunol 3, 583-590 (2002).

Giordano M, Marchetti C, Chiorboli E, Bona $\mathrm{G}$ and Momigliano Richiardi P. Evidence for gene conversion in the generation of extensive polymorphism in the promoter of the growth hormone gene. Hum Genet 100, 249-255 (1997).

Gorry MC, Gabbaizedeh D, Furey W, Gates LK Jr, Preston RA, Aston CE, Zhang Y, Ulrich C, Ehrlich GD and Whitcomb DC. Mutations in the cationic trypsinogen gene are associated with recurrent acute and chronic pancreatitis. Gastroenterology 113, 1063-1068 (1997).

Grady EF, Bohm SK and Bunnett NW. Turning off the signal: mechanisms that attenuate signaling by $\mathrm{G}$ protein-coupled receptors. Am J Physiol 273, G586-601 (1997).

Grishina Z, Ostrowska E, Halangk W, Sahin-Tóth M and Reiser G. Activity of recombinant trypsin isoforms on human proteinase-activated receptors (PAR): mesotrypsin cannot activate epithelial PAR-1, -2, but weakly activates brain PAR-1. Br J Pharmacol 146, 990-999 (2005).

Gudgeon AM, Heath DI Hurley P, Jehanli A, Patel G, Wilson C, Shenkin A, Austen $\mathrm{BM}$, Imrie CW and Hermon-Taylor J. Trypsinogen activation peptides assay in the early prediction of severity of acute pancreatitis. Lancet, 335, 4-8 (1990).

Guy O, Lombardo D, Bartelt DC, Amic J and Figarella C. Two human trypsinogens. Purification, molecular properties, and $\mathrm{N}$-terminal sequences. Biochemistry 17, 1669-1675 (1978).

Haglund C, Huhtala, ML, Halila H, Nordling S, Roberts PJ, Scheinin TM and Stenman UH. Tumour-associated trypsin inhibitor,
TATI, in patients with pancreatic cancer, pancreatitis and benign biliary diseases. Br.J Cancer 54, 297-303 (1986).

Halangk W, Kruger B, Ruthenburger M, Sturzebecher J, Albrecht E, Lippert H and Lerch MM. Trypsin activity is not involved in premature, intrapancreatic trypsinogen activation. Am J Physiol Gastrointest Liver Physiol 282, G367-74 (2002).

Halangk W, Lerch MM, Brandt-Nedelev B, Roth W, Ruthenbuerger M, Reinheckel T, Domschke W, Lippert H, Peters C and Deussing J. Role of cathepsin B in intracellular trypsinogen activation and the onset of acute pancreatitis. J Clin Invest 106, 773-781 (2000).

Halila H, Lehtovirta $\mathrm{P}$ and Stenman UH. Tumour-associated trypsin inhibitor (TATI) in ovarian cancer. Br J Cancer 57, 304-307 (1988).

Halila H, Huhtala ML, Schröder T, Kiviluoto $\mathrm{T}$ and Stenman UH. Pancreatic secretory trypsin inhibitor-like immunoreactivity in pancreatectomized patients. Clin Chim Acta, 153, 209-216 (1985).

Han B, Ji B and Logsdon CD. CCK independently activates intracellular trypsinogen and NF-kappaB in rat pancreatic acinar cells. Am J Physiol Cell Physiol 280, 465-472 (2001).

Hartwig W, Werner J, Warshaw AL, Antoniu B, Castillo CF, Gebhard MM, Uhl W and Buchler MW. Membrane-bound ICAM-1 is upregulated by trypsin and contributes to leukocyte migration in acute pancreatitis. Am J Physiol Gastrointest Liver Physiol 287, G1194-9 (2004).

Hasui Y, Suzumiya, J, Marutsuka, K, Sumiyoshi A, Hashida $\mathrm{S}$ and Ishikawa E. Comparative study of plasminogen activators in cancers and normal mucosae of human urinary bladder. Cancer Res 49, 1067-1070 (1989).

Haverback BJ, Dyce B, Bundy $\mathrm{H}$ and Edmondson HA. Trypsin, trypsinogen and trypsin inhibitor in human pancreatic juice. Am J Med 29, 421-433 (1960).

Hedström J, Kemppainen E, Andersen J, Jokela $\mathrm{H}$, Puolakkainen P and Stenman UH. A comparison of serum trypsinogen-2 and 
trypsin-2-alpha1-antitrypsin complex with lipase and amylase in the diagnosis and assessment of severity in the early phase of acute pancreatitis. Am J Gastroenterol 96, 424-430 (2001).

Hedström J, Haglund C, Kemppainen E, Leinimaa $\mathrm{M}$, Leinonen $\mathrm{J}$ and Stenman UH. Time-resolved immunofluorometric assay of trypsin-1 complexed with alpha(1)-antitrypsin in serum: increased immunoreactivity in patients with biliary tract cancer. Clin Chem 45, 1768-1773 (1999).

Hedström J, Haglund C, Haapiainen R and Stenman UH. Serum trypsinogen-2 and trypsin-2-alpha(1)-antitrypsin complex in malignant and benign digestive-tract diseases. Preferential elevation in patients with cholangiocarcinomas. Int $\mathrm{J}$ Cancer 66, 326-331 (1996a).

Hedström J, Korvuo A, Kenkimaki P, Tikanoja $\mathrm{S}$, Haapiainen R, Kivilaakso E. and Stenman UH. Urinary trypsinogen-2 test strip for acute pancreatitis. Lancet, 347, 729-730 (1996b).

Hedström J, Sainio V, Kemppainen E, Haapiainen R, Kivilaakso E, Schröder T, Leinonen J and Stenman UH. Serum complex of trypsin 2 and alpha 1 antitrypsin as diagnostic and prognostic marker of acute pancreatitis: clinical study in consecutive patients. Br Med J, 313, 333-337 (1996c).

Hedström J, Sainio V Kemppainen E, Puolakkainen P, Haapiainen R, Kivilaakso E, Schauman KO and Stenman UH. Urine trypsinogen-2 as marker of acute pancreatitis. Clin Chem 42, 685-690 (1996d).

Hedström J, Leinonen J, Sainio V and Stenman UH. Time-resolved immunofluorometric assay of trypsin- 2 complexed with alpha 1-antitrypsin in serum. Clin Chem 40, 1761-1765 (1994).

Hedstrom L. Serine protease mechanism and specificity. Chem Rev 102, 4501-4524 (2002).

Hedstrom L, Lin TY and Fast W. Hydrophobic interactions control zymogen activation in the trypsin family of serine proteases.
Biochemistry 35, 4515-4523 (1996).

Hedstrom L, Farr-Jones S, Kettner CA and Rutter WJ. Converting trypsin to chymotrypsin: ground-state binding does not determine substrate specificity. Biochemistry 33, 8764-8769 (1994a).

Hedstrom L, Perona JJ and Rutter WJ. Converting trypsin to chymotrypsin: residue 172 is a substrate specificity determinant. Biochemistry 33, 8757-8763 (1994b).

Hedstrom L, Szilagyi L and Rutter WJ. Converting trypsin to chymotrypsin: the role of surface loops. Science 255, 1249-1253 (1992).

Heimburger N, Heide K, Haupt $\mathrm{H}$ and Schultze HE. Analysis of the structure of human serum proteins. Clin.Chim.Acta, 10, 293-307 (1964).

Hemmilä I and Laitala V. Progress in lanthanides as luminescent probes. J Fluoresc, 15, 529-542 (2005).

Hemmilä, I Dakubu S Mukkala VM, Siitari $\mathrm{H}$ and Lövgren $\mathrm{T}$. Europium as a label in time-resolved immunofluorometric assays. Anal Biochem 137, 335-343 (1984).

Hermon-Taylor J, Perrin J, Grant DA, Appleyard A, Bubel M and Magee I. Immunofluorescent localisation of enterokinase in human small intestine. Gut, 18, 259-265 (1977).

Higashiyama, M, Monden T, Ogawa, M, Matsuura, N, Murotani M, Kawasaki Y, Tomita N, Murata, A, Shimano T and Mori T. Immunohistochemical study on pancreatic secretory trypsin inhibitor (PSTI) in gastric carcinomas. Am J Clin Pathol 93, 8-13 (1990a).

Higashiyama M, Monden T, Tomita N, Murotani M, Kawasaki Y, Morimoto H, Murata A, Shimano T, Ogawa M and Mori T. Expression of pancreatic secretory trypsin inhibitor (PSTI) in colorectal cancer. Br J Cancer 62, 954-958 (1990b).

Hille A, Braulke T, von Figura K and Huttner WB. Occurrence of tyrosine sulfate in proteins--a balance sheet. 1. Secretory and lysosomal proteins. Eur J Biochem 188, 577-586 (1990).

Hille A and Huttner WB. Occurrence of 
tyrosine sulfate in proteins--a balance sheet. 2. Membrane proteins. Eur J Biochem 188, 587-596 (1990).

Hirahara F, Miyagi E, Nagashima Y, Miyagi Y, Yasumitsu H, Koshikawa N, Nakatani Y, Nakazawa T, Udagawa K, Kitamura H, Minaguchi $\mathrm{H}$ and Miyazaki K. Differential expression of trypsin in human ovarian carcinomas and low-malignant-potential tumors. Gynecol Oncol 68, 162-165 (1998).

Hirahara F, Miyagi Y, Miyagi E, Yasumitsu H, Koshikawa N, Nagashima Y, Kitamura H, Minaguchi H, Umeda M and Miyazaki K. Trypsinogen expression in human ovarian carcinomas. Int $\mathrm{J}$ Cancer 63, 176-181 (1995).

Hirota $\mathrm{M}$, Ohmuraya $\mathrm{M}$ and Baba $\mathrm{H}$. The role of trypsin, trypsin inhibitor, and trypsin receptor in the onset and aggravation of pancreatitis. J Gastroenterol 41, 832-836 (2006a).

Hirota M, Ohmuraya M and Baba H. Genetic background of pancreatitis. Postgrad. Med.J, 82, 775-778 (2006b).

Hirota M, Kuwata K, Ohmuraya M and Ogawa M. From acute to chronic pancreatitis: the role of mutations in the pancreatic secretory trypsin inhibitor gene. JOP, 4 , 83-88 (2003).

Hochrainer K. and Lipp J. Ubiquitylation within signaling pathways in- and outside of inflammation. Thromb Haemost 97, 370-377 (2007).

Hoffhines AJ, Damoc E, Bridges KG, Leary JA and Moore KL. Detection and purification of tyrosine-sulfated proteins using a novel anti-sulfotyrosine monoclonal antibody. J Biol Chem, 281, 37877-37887 (2006).

Hoogenraad NJ and Wraight CJ. The effect of pristane on ascites tumor formation and monoclonal antibody production. Methods Enzymol 121, 375-381 (1986).

Horii A, Kobayashi T, Tomita N, Yamamoto, T, Fukushige S, Murotsu T, Ogawa M, Mori $\mathrm{T}$ and Matsubara K. Primary structure of human pancreatic secretory trypsin inhibitor (PSTI) gene. Biochem Biophys Res Commun 149, 635-641 (1987).

Hortin G, Folz, R, Gordon JI and Strauss
AW. Characterization of sites of tyrosine sulfation in proteins and criteria for predicting their occurrence. Biochem Biophys Res Commun 141, 326-333 (1986).

Hortin GL, Farries TC, Graham JP and Atkinson JP. Sulfation of tyrosine residues increases activity of the fourth component of complement. Proc Natl Acad Sci USA 86, 1338-1342 (1989).

Hotakainen K, Bjartell A, Sankila A, Järvinen $\mathrm{R}$, Paju A, Rintala E, Haglund $\mathrm{C}$ and Stenman UH. Differential expression of trypsinogen and tumor-associated trypsin inhibitor (TATI) in bladder cancer. Int J Oncol 28, 95-101 (2006).

Huang Q, Wang Z, Li Y, Liu S. and Tang Y. Refined $1.8 \AA$ resolution crystal structure of the porcine epsilon-trypsin. Biochim Biophys Acta 1209, 77-82 (1994).

Huber R, Kukla D, Bode W, Schwager P, Bartels K, Deisenhofer J and Steigemann W. Structure of the complex formed by bovine trypsin and bovine pancreatic trypsin inhibitor. II. Crystallographic refinement at $1.9 \AA$ resolution. J Mol Biol 89, 73-101 (1974).

Huhtala ML. Demonstration of a new acrosin inhibitor in human seminal plasma. Hoppe Seylers Z Physiol Chem, 365 819-825 (1984).

Huhtala ML, Kahanpää K, Seppälä M, Halila $\mathrm{H}$ and Stenman UH. Excretion of a tumorassociated trypsin inhibitor (TATI) in urine of patients with gynecological malignancy. Int J Cancer 31, 711-714 (1983).

Huhtala ML, Pesonen K, Kalkkinen N and Stenman UH. Purification and characterization of a tumor-associated trypsin inhibitor from the urine of a patient with ovarian cancer. J Biol Chem 257, 13713-13716 (1982).

Huntington JA, Read RJ and Carrell RW. Structure of a serpin-protease complex shows inhibition by deformation. Nature 407, 923-926 (2000).

Huttner WB. Sulphation of tyrosine residues-a widespread modification of proteins. Nature 299, 273-276 (1982).

Huttner WB. Protein tyrosine sulfation. Trends 
Biochem Sci 12, 361-363 (1987).

HyvonenMT,HerzigKH, Sinervirta R,Albrecht

E, Nordback I Sand J, Keinänen TA,

Vepsäläinen J, Grigorenko N, Khomutov

AR, Kruger B, Jänne J and Alhonen L.

Activated polyamine catabolism in acute pancreatitis: Alpha-methylated polyamine analogues prevent trypsinogen activation and pancreatitis-associated mortality. Am J Pathol 168, 115-122 (2006).

Ichikawa Y, Koshikawa N, Hasegawa S, Ishikawa T, Momiyama N, Kunizaki C, Takahashi M, Moriwaki Y, Akiyama H, Yamaoka H, Yanoma S, Tsuburaya A, Nagashima Y, Shimada H and Miyazaki $\mathrm{K}$. Marked increase of trypsin(ogen) in serum of linitis plastica (gastric cancer, borrmann 4) patients. Clin Cancer Res 6, 1385-1388 (2000).

Imai K, Yokohama Y, Nakanishi I, Ohuchi E, Fujii Y, Nakai N. and Okada Y. Matrix metalloproteinase 7 (matrilysin) from human rectal carcinoma cells. Activation of the precursor, interaction with other matrix metalloproteinases and enzymic properties. J Biol Chem 270, 6691-6697 (1995).

Imamura $\mathrm{T}$ and Kitamoto $\mathrm{Y}$. Expression of enteropeptidase in differentiated enterocytes, goblet cells, and the tumor cells in human duodenum. Am J Physiol Gastrointest Liver Physiol 285, G1235-1241 (2003).

Jang T, Uzbielo A, Sineff S, Naunheim R, Scott, MG and Lewis L. M. Point-of-care urine trypsinogen testing for the diagnosis of pancreatitis. Acad Emerg Med 14, 29-34 (2007).

Järvisalo J, Hakama M, Knekt P, Stenman UH, Leino A, Teppo L, Maatela J and Aromaa A. Serum tumor markers CEA, CA 50, TATI, and NSE in lung cancer screening. Cancer 71, 1982-1988 (1993).

Ji B, Bi Y, Simeone D, Mortensen RM and Logsdon CD. Human pancreatic acinar cells lack functional responses to cholecystokinin and gastrin. Gastroenterology 121, 1380-1390 (2001).

Jin X, Hirosaki T, Lin CY, Dickson RB, Higashi S, Kitamura $\mathrm{H}$ and Miyazaki K.
Production of soluble matriptase by human cancer cell lines and cell surface activation of its zymogen by trypsin. J Cell Biochem 95, 632-647 (2005).

Jones JG, Dodgson KS, Powell GM and Rose FA. Studies on $L$-tyrosine $O$-sulphate. 3 . The metabolic fate of the $L$-tyrosine $O\left[{ }^{35} \mathrm{~S}\right]$-sulphate residue of ${ }^{35} \mathrm{~S}$-labelled rabbit fibrinopeptide B. Biochem.J, 87, 548-553 (1963).

Jonsson P, Linder C, Genell S and Ohlsson K. Extrapancreatic origin of the pancreatic secretory trypsin inhibitor as an acutephase reactant. Pancreas 12, 303-307 (1996).

Josic D, Brown MK, Huang F, Lim YP, Rucevic M, Clifton JG and Hixson DC. Proteomic characterization of inter-alpha inhibitor proteins from human plasma. Proteomics 6, 2874-2885 (2006).

Jovov B, Berdiev BK, Fuller CM, Ji HL and Benos DJ. The serine protease trypsin cleaves $\mathrm{C}$ termini of beta- and gammasubunits of epithelial $\mathrm{Na}^{+}$channels. J Biol Chem 277, 4134-4140 (2002).

Kaslik G, Patthy A, Balint M and Graf L. Trypsin complexed with alpha 1-proteinase inhibitor has an increased structural flexibility. FEBS Lett 370, 179-183 (1995).

Kato Y, Nagashima Y, Koshikawa N, Miyagi Y, Yasumitsu $\mathrm{H}$ and Miyazaki K. Production of trypsins by human gastric cancer cells correlates with their malignant phenotype. Eur.J Cancer 34, 1117-1123 (1998).

Katona G, Berglund G. I, Hajdu J, Graf L. and Szilagyi L. Crystal structure reveals basis for the inhibitor resistance of human brain trypsin. J Mol Biol 315, 1209-1218 (2002).

Kawabata A, Kuroda R, Nishida M, Nagata N, Sakaguchi Y, Kawao, N, Nishikawa H, Arizono N and Kawai K. Protease-activated receptor-2 (PAR-2) in the pancreas and parotid gland: Immunolocalization and involvement of nitric oxide in the evoked amylase secretion. Life Sci 71, 2435-2446 (2002).

Kawabata A, Nishikawa H, Kuroda R, Kawai $\mathrm{K}$ and Hollenberg MD. Proteinase- 
activated receptor-2 (PAR-2): regulation of salivary and pancreatic exocrine secretion in vivo in rats and mice. Br J Pharmacol 129, 1808-1814 (2000).

Kawano N, Osawa H, Ito T, Nagashima Y, Hirahara F, Inayama Y, Nakatani Y, Kimura S, Kitajima H, Koshikawa N, Miyazaki K. and Kitamura H. Expression of gelatinase A, tissue inhibitor of metalloproteinases-2, matrilysin, and trypsin(ogen) in lung neoplasms: an immunohistochemical study. Hum Pathol 28, 613-622 (1997).

Kazal LA, Spicer DS. and Brahinsky RA. Isolation of a crystalline trypsin inhibitoranticoagulant protein from the pancreas. $\mathrm{J}$ Am Chem Soc 70, 437-444 (1948).

Kehoe JW, Velappan N, Walbolt M, Rasmussen J, King D, Lou J, Knopp, K, Pavlik, P, Marks JD, Bertozzi CR and Bradbury AR. Using phage display to select antibodies recognizing posttranslational modifications independently of sequence context. Mol Cell Proteomics 5, 2350-2363 (2006).

Kehoe JW and Bertozzi CR. Tyrosine sulfation: a modulator of extracellular protein-protein interactions. Chem Biol 7, R57-61 (2000).

Keiles S and Kammesheidt A. Identification of CFTR, PRSS1, and SPINK1 mutations in 381 patients with pancreatitis. Pancreas 33, 221-227 (2006).

Keller PJ and Allan BJ. The protein composition of human pancreatic juice. $\mathrm{J}$ Biol Chem 242, 281-287 (1967).

Kelloniemi E, Rintala E, Finne P, Stenman UH and Finnbladder Group. Tumorassociated trypsin inhibitor as a prognostic factor during follow-up of bladder cancer. Urology 62, 249-253 (2003).

Kemppainen EA, Hedström, JI, Puolakkainen PA, Sainio VS, Haapiainen RK, Perhoniemi V, Osman S, Kivilaakso EO and Stenman UH. Rapid measurement of urinary trypsinogen- 2 as a screening test for acute pancreatitis. N Engl J Med 336, 1788-1793 (1997).

Kenesi E, Katona G. and Szilagyi L. Structural and evolutionary consequences of unpaired cysteines in trypsinogen. Biochem Biophys
Res Commun 309, 749-754 (2003).

Kerr MA, Walsh,KA and Neurath H. Catalysis by serine proteases and their zymogens. A study of acyl intermediates by circular dichroism. Biochemistry 14, 5088-5094 (1975).

Kikuchi N, Nagata K, Shin M, Mitsushima K, Teraoka H and Yoshida N. Site-directed mutagenesis of human pancreatic secretory trypsin inhibitor. J Biochem (Tokyo) 106, 1059-1063 (1989).

Kikuchi N, Nagata K, Yoshida N and Ogawa $\mathrm{M}$. The multiplicity of human pancreatic secretory trypsin inhibitor. J Biochem (Tokyo) 98, 687-694 (1985).

Kimland M, Russick C, Marks WH and Borgström A. Immunoreactive anionic and cationic trypsin in human serum. Clin. Chim.Acta 184, 31-46 (1989).

Kiraly O, Boulling A, Witt, H, Le Maréchal C, Chen JM, Rosendahl J, Battaggia C, Wartmann T, Sahin-Tóth, $M$ and Feréc C. Signal peptide variants that impair secretion of pancreatic secretory trypsin inhibitor (SPINK1) cause autosomal dominant hereditary pancreatitis. Hum Mutat 28, 469-476 (2007).

Kitahara T, Takatsuka Y, Fujimoto KI, Tanaka S, Ogawa $\mathrm{M}$ and Kosaki $\mathrm{G}$. Radioimmunoassay for human pancreatic secretory trypsin inhibitor: measurement of serum pancreatic secretory trypsin inhibitor in normal subjects and subjects with pancreatic diseases. Clin Chim Acta 103, 135-143 (1980).

Kitamoto, Y, Yuan X, Wu Q, McCourt, DW and Sadler JE. Enterokinase, the initiator of intestinal digestion, is a mosaic protease composed of a distinctive assortment of domains. Proc Natl Acad Sci USA 91, 7588-7592 (1994).

Klonowski-Stumpe H, Luthen R, Han B, Sata N, Haussinger D and Niederau C. Inhibition of cathepsin B does not affect the intracellular activation of trypsinogen by cerulein hyperstimulation in isolated rat pancreatic acinar cells. Pancreas 16, 96-101 (1998).

Kloppel G. Toward a new classification of chronic pancreatitis. J Gastroenterol 42 
Suppl 17, 55-57 (2007).

Kloppel G and Maillet B. Pathology of acute and chronic pancreatitis. Pancreas 8, 659-670 (1993).

Kohler $\mathrm{G}$ and Milstein C. Continuous cultures of fused cells secreting antibody of predefined specificity. Nature 256, 495-497 (1975).

Koivunen E, Ristimaki A, Itkonen O, Osman S, Vuento, M and Stenman UH. Tumorassociated trypsin participates in cancer cell-mediated degradation of extracellular matrix. Cancer Res 51, 2107-2112 (1991a).

Koivunen E, Saksela O, Itkonen O, Osman S, Huhtala ML and Stenman UH. Human colon carcinoma, fibrosarcoma and leukemia cell lines produce tumorassociated trypsinogen. Int J Cancer 47, 592-596 (1991b).

Koivunen E, Huhtala ML and Stenman UH. Human ovarian tumor-associated trypsin. Its purification and characterization from mucinous cyst fluid and identification as an activator of pro-urokinase. J Biol Chem 264, 14095-14099 (1989).

Kong W, McConalogue K, Khitin LM, Hollenberg MD, Payan DG, Bohm SK and Bunnett, N. W. Luminal trypsin may regulate enterocytes through proteinaseactivated receptor 2. Proc Natl Acad Sci USA 94, 8884-8889 (1997).

Kool ET. Hydrogen bonding, base stacking, and steric effects in dna replication. Annu Rev Biophys BioMol Struct, 30, 1-22 (2001).

Koshikawa N, Hasegawa S, Nagashima Y, Mitsuhashi K, Tsubota Y, Miyata S Miyagi Y, Yasumitsu H and Miyazaki K. Expression of trypsin by epithelial cells of various tissues, leukocytes, and neurons in human and mouse. Am J Pathol 153, 937-944 (1998).

Koshikawa N, Nagashima Y, Miyagi Y, Mizushima H, Yanoma S, Yasumitsu H and Miyazaki K. Expression of trypsin in vascular endothelial cells. FEBS Lett 409, 442-448 (1997).

Koshikawa N Yasumitsu H, Nagashima Y, Umeda $\mathrm{M}$ and Miyazaki K. Identification of one- and two-chain forms of trypsinogen 1 produced by a human gastric adenocarcinoma cell line. Biochem J 303, 187-190 (1994).

Koshikawa N, Yasumitsu H, Umeda M and Miyazaki K. Multiple secretion of matrix serine proteinases by human gastric carcinoma cell lines. Cancer Res 52, 5046-5053 (1992).

Kossiakoff AA and Spencer SA. Direct determination of the protonation states of aspartic acid-102 and histidine-57 in the tetrahedral intermediate of the serine proteases: neutron structure of trypsin. Biochemistry 20, 6462-6474 (1981).

Kossiakoff AA and Spencer SA. Neutron diffraction identifies His 57 as the catalytic base in trypsin. Nature 288, 414-416 (1980).

Kossiakoff AA, Chambers JL, Kay LM and Stroud RM. Structure of bovine trypsinogen at $1.9 \AA$ resolution. Biochemistry 16, 654-664 (1977).

Krapp A, Knofler M, Frutiger S, Hughes GJ, Hagenbuchle $\mathrm{O}$ and Wellauer PK. The p48 DNA-binding subunit of transcription factor PTF1 is a new exocrine pancreasspecific basic helix-loop-helix protein. EMBO J, 15, 4317-4329 (1996).

Kraut J. Serine proteases: structure and mechanism of catalysis. Annu Rev Biochem 46, 331-358 (1977).

Krem MM and Di Cera E. Conserved water molecules in the specificity pocket of serine proteases and the molecular mechanism of $\mathrm{Na}^{+}$binding. Proteins 30, 34-42 (1998).

Krishtal O. The ASICs: signaling molecules? Modulators? Trends Neurosci 26, 477-483 (2003).

Kryger G, Silman I and Sussman JL. Structure of acetylcholinesterase complexed with E2020 (Aricept): implications for the design of new anti-Alzheimer drugs. Structure 7, 297-307 (1999).

Kryger G, Silman I and Sussman JL. Threedimensional structure of a complex of E2020 with acetylcholinesterase from Torpedo californica. J Physiol.Paris 92, 191-194 (1998).

Kühne W. Ueber das Verhalten verschiedener 
organisirter und sog. ungeformter Fermente. FEBS Letters 62, E4-E7 (1976).

Kukor Z, Tóth M and Sahin-Tóth M. Human anionic trypsinogen: properties of autocatalytic activation and degradation and implications in pancreatic diseases. Eur J Biochem 270, 2047-2058 (2003).

Kukor, Z, Mayerle J, Kruger B, Tóth M, Steed PM, Halangk W, Lerch MM and SahinTóth M. Presence of cathepsin B in the human pancreatic secretory pathway and its role in trypsinogen activation during hereditary pancreatitis. J Biol Chem 277, 21389-21396 (2002a).

Kukor Z, Tóth M, Pal G and Sahin-Tóth M. Human cationic trypsinogen. $\operatorname{Arg}(117)$ is the reactive site of an inhibitory surface loop that controls spontaneous zymogen activation. J Biol Chem 277, 6111-6117 (2002b).

Kunitz $\mathrm{M}$ and Northrop JH. Isolation from beef pancreas of crystalline trypsinogen, trypsin, a trypsin inhibitor, and an inhibitor-trypsin compound. J Gen Physiol 19, 991-1007 (1935).

Kunitz $\mathrm{M}$ and Northrop JH. Autocatalytic activation of trypsinogen in the presence of concentrated ammonium or magnesium sulfate. Science 80, 190 (1934).

Kuwata K, Hirota M, Shimizu H, Nakae M, Nishihara S, Takimoto, A, Mitsushima K, Kikuchi N, Endo, K, Inoue M and Ogawa M. Functional analysis of recombinant pancreatic secretory trypsin inhibitor protein with amino-acid substitution. J Gastroenterol 37, 928-934 (2002).

Kylänpää-Bäck M, Kemppainen E, Puolakkainen P, Hedström J, Haapiainen R, Perhoniemi V, Kivilaakso E, Korvuo $A$ and Stenman U. Reliable screening for acute pancreatitis with rapid urine trypsinogen-2 test strip. Br J Surg 87, 49-52 (2000).

Kylänpää-Bäck ML, Kemppainen E, Puolakkainen P, Hedström, J, Haapiainen R, Korvuo, A and Stenman UH. Comparison of urine trypsinogen-2 test strip with serum lipase in the diagnosis of acute pancreatitis. HepatoGastroenterology 49,
1130-1134 (2002).

Laemmli UK. Cleavage of structural proteins during the assembly of the head of bacteriophage T4. Nature 227, 680-685 (1970).

Lafont P, Guy-Crotte O, Paulin C, Galvain $\mathrm{D}$, Mertani S, Figarella $\mathrm{C}$ and Barbier Y. A specific immunoradiometric assay of cationic trypsin(ogen) that does not recognize trypsin-alpha-1-proteinase inhibitor complex. Clin.Chim.Acta 235, 197-206 (1995).

Largman C, Brodrick, JW, Geokas MC and Johnson JH. Demonstration of human pancreatic anionic trypsinogen in normal serum by radioimmunoassay. Biochim Biophys Acta 543, 450-454 (1978).

Laskowski $\mathrm{M}$ and Wu FC. Temporary inhibition of trypsin. J Biol Chem 204, 797-805 (1953).

Laskowski M Jr and Kato I. Protein inhibitors of proteinases. Annu Rev Biochem, 49, 593-626 (1980).

Lasson A, Borgström A and Ohlsson K. Elevated pancreatic secretory trypsin inhibitor levels during severe inflammatory disease, renal insufficiency, and after various surgical procedures. Scand J Gastroenterol 21, 1275-1280 (1986).

Le Maréchal C, Chen JM, Le Gall C, Plessis G, Chipponi J, Chuzhanova NA Raguenes O and Feréc C. Two novel severe mutations in the pancreatic secretory trypsin inhibitor gene (SPINK1) cause familial and/or hereditary pancreatitis. Hum.Mutat, 23, 205 (2004).

Le MaréchalC, Bretagne JF, Raguenes O, Quere I, Chen JM and Feréc C. Identification of a novel pancreatitis-associated missense mutation, R116C, in the human cationic trypsinogen gene (PRSS1). Mol Genet Metab 74, 342-344 (2001).

Le Maréchal C, Masson E, Jian-Min C, Morel F, Ruszniewski $\mathrm{P}$, Levy $\mathrm{P}$ and Férec C. Hereditary pancreatitis caused by triplication of the trypsinogen locus. Nat Genet 38, 1372-1374 (2006).

Le Moine O, Devaster JM, Deviere J, Thiry P, Cremer $\mathrm{M}$ and Ooms HA. Trypsin activity. A new marker of acute alcoholic 
pancreatitis. Dig Dis Sci 39, 2634-2638 (1994).

Le TQ, Kawachi M, Yamada H, Shiota M, Okumura Y and Kido H. Identification of trypsin I as a candidate for influenza A virus and Sendai virus envelope glycoprotein processing protease in rat brain. Biol Chem, 387, 467-475 (2006).

Lee JW, Yong Song S, Choi JJ, Lee SJ, Kim BG, Park CS, Lee JH, Lin CY, Dickson RB and Bae DS. Increased expression of matriptase is associated with histopathologic grades of cervical neoplasia. Hum Pathol 36, 626-633 (2005).

Lee RW and Huttner WB. $\left(\mathrm{Glu}^{62}, \mathrm{Ala}^{30}, \mathrm{Tyr}^{8}\right)$ serves as high-affinity substrate for tyrosylprotein sulfotransferase: a Golgi enzyme. Proc Natl Acad Sci USA 82, 6143-6147 (1985).

Lee RW and Huttner WB. Tyrosine- $O$-sulfated proteins of PC12 pheochromocytoma cells and their sulfation by a tyrosylprotein sulfotransferase. J Biol Chem 258, 11326-11334 (1983).

Lee SL, Dickson RB and Lin CY. Activation of hepatocyte growth factor and urokinase/ plasminogen activator by matriptase, an epithelial membrane serine protease. J Biol Chem 275, 36720-36725 (2000).

Lee SP, Nicholls JF and Park HZ. Biliary sludge as a cause of acute pancreatitis. N Engl J Med 326, 589-593 (1992).

Lee YC, Pan HW, Peng SY, Lai PL, Kuo WS, $\mathrm{Ou} \mathrm{YH}$ and Hsu HC. Overexpression of tumour-associated trypsin inhibitor (TATI) enhances tumour growth and is associated with portal vein invasion, early recurrence and a stage-independent prognostic factor of hepatocellular carcinoma. Eur J Cancer 43, 736-744 (2007).

Leiros HK Schroder, Brandsdal BO, Andersen OA, Os V, Leiros I, Helland R, Otlewski J, Willassen NP and Smalås AO. Trypsin specificity as elucidated by LIE calculations, X-ray structures, and association constant measurements. Protein Sci 13, 1056-1070 (2004).

Lempinen $M$, Isoniemi $H$, Mäkisalo, $H$, Nordin A, Halme L, Arola J, Höckerstedt, $\mathrm{K}$ and Stenman UH. Enhanced detection of cholangiocarcinoma with serum trypsinogen-2 in patients with severe bile duct strictures. J Hepatol 47, 677-683 (2007).

Lerch MM, Saluja A K, Dawra R, Saluja M and Steer M L. The effect of chloroquine administration on two experimental models of acute pancreatitis. Gastroenterology 104, 1768-1779 (1993).

Leyte A, van Schijndel HB, Niehrs C, Huttner WB, Verbeet MP, Mertens $\mathrm{K}$ and van Mourik JA. Sulfation of Tyr1680 of human blood coagulation factor VIII is essential for the interaction of factor VIII with von Willebrand factor. J Biol Chem 266, 740-746 (1991).

Li S and Shang Y. Regulation of SRC family coactivators by post-translational modifications. Cell Signal 19, 1101-1112 (2007).

Lieberman JM, Marks WH, Stuart FP, Abecassis MM, Florence LS and Kauffman D. Co-monitoring serum anodal trypsinogen, serum amylase, and serum creatinine accurately differentiates rejection from other causes of allograft dysfunction after simultaneous pancreaskidney transplantation. Transplant Proc 29, 676-677 (1997).

Light $A$ and Janska H. Enterokinase (enteropeptidase): comparative aspects. Trends Biochem Sci 14, 110-112 (1989).

Lilja H. A kallikrein-like serine protease in prostatic fluid cleaves the predominant seminal vesicle protein. J Clin.Invest, 76, 1899-1903 (1985).

Lindkvist B, Fajardo I, Pejler $G$ and Borgström A. Cathepsin B activates human trypsinogen 1 but not proelastase 2 or procarboxypeptidase B. Pancreatology 6, 224-231 (2006).

Liotta LA and KohnEC. The microenvironment of the tumour-host interface. Nature 411, 375-379 (2001).

Liotta LA, Tryggvason K, Garbisa S, Hart I, Foltz CM and Shafie S. Metastatic potential correlates with enzymatic degradation of basement membrane collagen. Nature 284, 67-68 (1980).

Logsdon C. Phosphatidylinositol 3-kinase and 
trypsin activation in pancreatitis. J Clin Invest 108, 1267-1268 (2001).

Lowenfels A B, Maisonneuve P, DiMagno EP, Elitsur Y, Gates LK Jr, Perrault J, Whitcomb DC and the International Hereditary Pancreatitis Study Group. Hereditary pancreatitis and the risk of pancreatic cancer. J Natl Cancer Inst 89, 442-446 (1997).

Ludeman MJ, Zheng YW, Ishii $\mathrm{K}$ and Coughlin SR. Regulated shedding of PAR1 N-terminal exodomain from endothelial cells. J Biol Chem 279, 18592-18599 (2004).

Lukkonen A, Sorsa T, Salo, T, Tervahartiala $\mathrm{T}$, Koivunen E, Golub, L, Simon S and Stenman UH. Down-regulation of trypsinogen- 2 expression by chemically modified tetracyclines: association with reduced cancer cell migration. Int J Cancer 86, 577-581 (2000).

Lukkonen A, Lintula S, von Boguslawski K, Carpen O, Ljungberg, B, Landberg $G$ and Stenman UH. Tumor-associated trypsin inhibitor in normal and malignant renal tissue and in serum of renal-cell carcinoma patients. Int J Cancer 83, 486-490 (1999).

Lyon M, Rushton G, Askari JA, Humphries MJ and Gallagher JT. Elucidation of the structural features of heparan sulfate important for interaction with the Hep-2 domain of fibronectin. J Biol Chem 275, 4599-4606 (2000).

Ma W, Tang C and Lai L. Specificity of trypsin and chymotrypsin: loop-motion-controlled dynamic correlation as a determinant. Biophys J 89, 1183-1193 (2005).

Macfarlane SR, Seatter MJ, Kanke T, Hunter GD and Plevin R. Proteinase-activated receptors. Pharmacol.Rev, 53, 245-282 (2001).

Maeda K, Hirota M, Kimura Y, Ichihara A, Ohmuraya M, Sugita $\mathrm{H}$ and Ogawa M. Proinflammatory role of trypsin and protease-activated receptor- 2 in a rat model of acute pancreatitis. Pancreas 31, 54-62 (2005).

Mahurkar S, Idris MM, Reddy DN, Bhaskar S, Rao GV, Thomas V, Singh L and Chandak GR. Association of cathepsin B gene polymorphisms with tropical calcific pancreatitis. Gut 55, 1270-1275 (2006).

Malhotra S, Newman E, Eisenberg D, Scholes J, Wieczorek, R, Mignatti P and Shamamian P. Increased membrane type 1 matrix metalloproteinase expression from adenoma to colon cancer: a possible mechanism of neoplastic progression. Dis Colon Rectum 45, 537-543 (2002).

Mallory PA and Travis J. Inhibition spectra of the human pancreatic endopeptidases. Am J Clin Nutr 28, 823-830 (1975).

Mallory PA and Travis J. Human pancreatic enzymes. Characterization of anionic human trypsin. Biochemistry 12, 2847-2851 (1973).

Marchbank, T, Freeman TC and Playford RJ. Human pancreatic secretory trypsin inhibitor. Distribution, actions and possible role in mucosal integrity and repair. Digestion 59, 167-174 (1998).

Marchbank T, Chinery R, Hanby AM, Poulsom $\mathrm{R}$, Elia $\mathrm{G}$ and Playford RJ. Distribution and expression of pancreatic secretory trypsin inhibitor and its possible role in epithelial restitution. Am J Pathol 148, 715-722 (1996).

Marks WH, Borgström A, Sollinger H, Marks $\mathrm{C}$ and Lorber MI. Serum anodal trypsinogen is a predictive biochemical marker for pancreas allograft rejection. Transplant Proc 22, 673-674 (1990).

Marks W H. and Ohlsson K. Elimination of pancreatic secretory trypsin inhibitor from the circulation. A study in man. Scand J Gastroenterol 18, 955-959 (1983).

Masson E, Le Maréchal C, Chen JM, Frebourg, T, Lerebours E and Feréc C. Detection of a large genomic deletion in the pancreatic secretory trypsin inhibitor (SPINK1) gene. Eur J Hum Genet, 14, 1204-1208 (2006).

Matsuda K, Ogawa M, Shibata T, Nishibe S, Miyauchi K, Matsuda Y and Mori T. Postoperative elevation of serum pancreatic secretory trypsin inhibitor. Am J Gastroenterol 80, 694-698 (1985).

McDowell RS and Kossiakoff AA. A comparison of neutron diffraction and molecular dynamics structures: hydroxyl group and water molecule orientations in 
trypsin. J Mol Biol 250, 553-570 (1995).

McNamee R, Braganza JM, Hogg J, Leck

I, Rose P and Cherry NM. Occupational exposure to hydrocarbons and chronic pancreatitis: a case-referent study. Occup Environ Med 51, 631-637 (1994).

Medveczky P, Antal J, Patthy A, Kekesi K, Juhasz G, Szilagyi L and Graf L. Myelin basic protein, an autoantigen in multiple sclerosis, is selectively processed by human trypsin 4. FEBS Lett 580, 545-552 (2006).

Michnick DA, Pittman DD, Wise RJ and Kaufman RJ. Identification of individual tyrosine sulfation sites within factor VIII required for optimal activity and efficient thrombin cleavage. J Biol Chem 269, 20095-20102 (1994).

Mignatti $P$ and Rifkin DB. Biology and biochemistry of proteinases in tumor invasion. Physiol Rev 73, 161-195 (1993).

Mignatti P, Robbins E and Rifkin DB. Tumor invasion through the human amniotic membrane: requirement for a proteinase cascade. Cell 47, 487-498 (1986).

Minn A, Schubert M, Neiss WF and MullerHill B. Enhanced GFAP expression in astrocytes of transgenic mice expressing the human brain-specific trypsinogen IV. Glia 22, 338-347 (1998).

Mitchell JB, Nandi CL, McDonald IK, Thornton JMand Price SL.Amino/aromatic interactions in proteins: is the evidence stacked against hydrogen bonding? J Mol Biol 239, 315-331 (1994).

Miyagi E, Yasumitsu H, Hirahara F, Minaguchi $\mathrm{H}$, Koshikawa N, Miyazaki K and Umeda M. Characterization of matrix-degrading proteinases and their inhibitors secreted by human gynecological carcinoma cells. Jpn J Cancer Res 86, 568-576 (1995).

Miyata S, Koshikawa N, Yasumitsu H and Miyazaki K. Trypsin stimulates integrin $\alpha_{5} \beta_{1}$-dependent adhesion to fibronectin and proliferation of human gastric carcinoma cells through activation of proteinaseactivated receptor-2. J Biol Chem 275, 4592-4598 (2000).

Miyata S, Koshikawa N, Higashi S, Miyagi
Y, Nagashima Y, Yanoma S, Kato Y, Yasumitsu $\mathrm{H}$ and Miyazaki K. Expression of trypsin in human cancer cell lines and cancer tissues and its tight binding to soluble form of Alzheimer amyloid precursor protein in culture. J Biochem (Tokyo) 125, 1067-1076 (1999).

Miyata S, Miyagi Y, Koshikawa N, Nagashima Y, Kato Y, Yasumitsu H, Hirahara F, Misugi $\mathrm{K}$ and Miyazaki K. Stimulation of cellular growth and adhesion to fibronectin and vitronectin in culture and tumorigenicity in nude mice by overexpression of trypsinogen in human gastric cancer cells. Clin Exp Metastasis 16, 613-622 (1998).

Moilanen M, Sorsa T, Stenman M, Nyberg P, Lindy O, Vesterinen J, Paju A, Konttinen YT, Stenman UH and Salo T. Tumorassociated trypsinogen-2 (trypsinogen-2) activates procollagenases (MMP-1, -8, -13) and stromelysin-1 (MMP-3) and degrades type I collagen. Biochemistry 42, 5414-5420 (2003).

Molino M, Barnathan E. S, Numerof, R, Clark, J, Dreyer M, Cumashi A, Hoxie JA, Schechter N, Woolkalis M and Brass LF. Interactions of mast cell tryptase with thrombin receptors and PAR-2. J Biol Chem 272, 4043-4049 (1997).

Molloy SS, Bresnahan PA, Leppla SH, Klimpel KR. and Thomas G. Human furin is a calcium-dependent serine endoprotease that recognizes the sequence Arg-X-XArg and efficiently cleaves anthrax toxin protective antigen. J Biol Chem 267, 16396-16402 (1992).

Monigatti F, Gasteiger E, Bairoch A and Jung E. The Sulfinator: predicting tyrosine sulfation sites in protein sequences. Bioinformatics 18, 769-770 (2002).

Moore KL. The biology and enzymology of protein tyrosine $O$-sulfation. J Biol Chem 278, 24243-24246 (2003).

Nathan JD and Liddle RA. Neurohormonal control of pancreatic exocrine secretion. Miscellaneous Article. Curr Opin Gastroenterol 18, 536-544 (2002).

Nemeth AL, Medveczky P, Tóth J, Siklodi E, Schlett K, Patthy A, Palkovits M, Ovadi J, Tokesi N, Nemeth, P, Szilagyi L and Graf 
L. Unconventional translation initiation of human trypsinogen 4 at a CUG codon with an N-terminal leucine. A possible means to regulate gene expression. FEBS Journal 274, 1610-1620 (2007).

Nemeth-Cawley JF, Karnik S and Rouse JC. Analysis of sulfated peptides using positive electrospray ionization tandem mass spectrometry. J Mass Spectrom 36, 1301-1311 (2001).

Nemoda Z and Sahin-Tóth M. Chymotrypsin $\mathrm{C}$ (caldecrin) stimulates autoactivation of human cationic trypsinogen. J Biol Chem 281, 11879-11886 (2006).

Nemoda $Z$ and Sahin-Tóth M. The tetraaspartate motif in the activation peptide of human cationic trypsinogen is essential for autoactivation control but not for enteropeptidase recognition. J Biol Chem 280, 29645-29652 (2005).

Neurath H. and Walsh KA. Role of proteolytic enzymes in biological regulation. Proc Natl Acad Sci USA 73, 3825-3832 (1976).

Nguyen TD, Moody MW, Steinhoff M, Okolo C, Koh DS. and Bunnett, N. W. Trypsin activates pancreatic duct epithelial cell ion channels through proteinase-activated receptor-2. J Clin Invest 103, 261-269 (1999).

Niehrs $\mathrm{C}$ and Huttner WB. Purification and characterization of tyrosylprotein sulfotransferase. EMBO J 9, 35-42 (1990).

Niehrs C, Kraft M, Lee RW and Huttner WB. Analysis of the substrate specificity of tyrosylprotein sulfotransferase using synthetic peptides. J Biol Chem 265, 8525-8532 (1990).

Nishimura H, Kakizaki I, Muta T, Sasaki N, $\mathrm{Pu} \mathrm{PX}$, Yamashita $\mathrm{T}$ and Nagasawa $\mathrm{S}$. cDNA and deduced amino acid sequence of human PK-120, a plasma kallikreinsensitive glycoprotein. FEBS Lett 357, 207-211 (1995).

Nitta T, Igarashi K. and Yamamoto N. Polyamine depletion induces apoptosis through mitochondria-mediated pathway. Exp Cell Res 276, 120-128 (2002).

Northrop, John H. and Kunitz, M. Crystalline trypsin: I. Isolation and tetsts of purity. J
Gen. Physiol 16, 267-294 (1932).

Nyaruhucha CN, Kito $\mathrm{M}$ and Fukuoka SI. Identification and expression of the cDNAencoding human mesotrypsin(ogen), an isoform of trypsin with inhibitor resistance. J Biol Chem 272, 10573-10578 (1997).

Nyberg P, Ylipalosaari M, Sorsa T and Salo T. Trypsins and their role in carcinoma growth. Exp Cell Res 312, 1219-1228 (2006).

Nyberg P, Xie L and Kalluri R. Endogenous inhibitors of angiogenesis. Cancer Res 65, 3967-3979 (2005).

Nyberg P, Moilanen M, Paju A, Sarin A, Stenman UH, Sorsa T and Salo T. MMP-9 activation by tumor trypsin- 2 enhances in vivo invasion of human tongue carcinoma cells. J Dent Res 81, 831-835 (2002).

Nystedt S, Emilsson K, Larsson AK, Strombeck B and Sundelin J. Molecular cloning and functional expression of the gene encoding the human proteinaseactivated receptor 2. Eur J Biochem 232, 84-89 (1995).

Nystedt S, Emilsson K, Wahlestedt C and Sundelin J. Molecular cloning of a potential proteinase activated receptor. Proc Natl Acad Sci USA 91, 9208-9212 (1994).

Obst U, Banner DW, Weber L and Diederich F. Molecular recognition at the thrombin active site: structure-based design and synthesis of potent and selective thrombin inhibitors and the X-ray crystal structures of two thrombin-inhibitor complexes. Chem Biol 4, 287-295 (1997).

Ockenga J, Stuhrmann M, Ballmann M, Teich N, Keim V, Dork $\mathrm{T}$ and Manns MP. Mutations of the cystic fibrosis gene, but not cationic trypsinogen gene, are associated with recurrent or chronic idiopathic pancreatitis. Am J Gastroenterol 95, 2061-2067 (2000).

Ogawa M, Shibata T, Niinobu T, Uda K, Takata $\mathrm{N}$ and Mori T. Serum pancreatic secretory trypsin inhibitor (PSTI) in patients with inflammatory diseases. Adv Exp Med Biol 240, 505-508 (1988).

Ogawa M, Matsuda K, Shibata T, Matsuda Y, Ukai T, Ohta M and Mori T. Elevation of serum pancreatic secretory trypsin inhibitor 
(PSTI) in patients with serious injury. Res Commun Chem Pathol Pharmacol 50, 259-266 (1985).

Ohmachi Y, Murata A, Matsuura N, Yasuda T, Yasuda T, Monden M, Mori T, Ogawa M and Matsubara K. Specific expression of the pancreatic-secretory-trypsin-inhibitor (PSTI) gene in hepatocellular carcinoma. Int J Cancer 55, 728-734 (1993).

Ohmuraya M, Hirota M, Araki K, Baba H. and Yamamura K. Enhanced trypsin activity in pancreatic acinar cells deficient for serine protease inhibitor kazal type 3 . Pancreas 33, 104-106 (2006).

Ohmuraya M, Hirota M, Araki M, Mizushima $\mathrm{N}$, Matsui M, Mizumoto, T, Haruna K, Kume S, Takeya M, Ogawa M, Araki K and Yamamura K. Autophagic cell death of pancreatic acinar cells in serine protease inhibitor Kazal type 3-deficient mice. Gastroenterology 129, 696-705 (2005).

Ohta T, Tajima H, Fushida S, Kitagawa H, Kayahara M, Nagakawa T, Miwa K, Yamamoto M, Numata M, Nakanuma Y, Kitamura Y. and Terada T. Cationic trypsinogen produced by human pancreatic ductal cancer has the characteristics of spontaneous activation and gelatinolytic activity in the presence of proton. Int J Mol Med 1, 689-692 (1998).

Oikonomopoulou K, Hansen KK, Saifeddine M, Tea I, Blaber M, Blaber SI, Scarisbrick I, Andrade-Gordon P, Cottrell GS, Bunnett NW, Diamandis EP and Hollenberg MD. Proteinase-activated receptors, targets for kallikrein signaling. J Biol Chem 281, 32095-32112 (2006).

O'Keefe SJ, Lee RB, Li J, Zhou W, Stoll B and Dang Q. Trypsin and splanchnic protein turnover during feeding and fasting in human subjects. Am J Physiol Gastrointest Liver Physiol 290, G213-21 (2006).

Omary MB, Ku NO, Tao GZ, Toivola DM and Liao J. "Heads and tails" of intermediate filament phosphorylation: multiple sites and functional insights. Trends Biochem Sci 31, 383-394 (2006).

Ouchterlony, O. Diffusion-in-gel methods for immunological analysis. Prog Allergy Vol. 5, 1-78 (1958).
Ouyang YB, Crawley JT, Aston CE and Moore KL. Reduced body weight and increased postimplantation fetal death in tyrosylprotein sulfotransferase-1-deficient mice. J Biol Chem 277, 23781-23787 (2002).

Ouyang YB and Moore KL. Molecular cloning and expression of human and mouse tyrosylprotein sulfotransferase- 2 and a tyrosylprotein sulfotransferase homologue in Caenorhabditis elegans. J Biol Chem 273, 24770-24774 (1998).

Ouyang Ying-bin, Lane William S and Moore Kevin L. Tyrosylprotein sulfotransferase: Purification and molecular cloning of an enzyme that catalyzes tyrosine $O$-sulfation, a common posttranslational modification of eukaryotic proteins. Proc Natl Acad Sci USA 95, 2896-2901 (1998).

Owyang C and Logsdon CD. New insights into neurohormonal regulation of pancreatic secretion. Gastroenterology 127, 957-969 (2004).

Owyang C. Physiological mechanisms of cholecystokinin action on pancreatic secretion. Am J Physiol 271, G1-7 (1996).

Oyama K, Ohta T, Nishimura GI, Elnemr A, Yasui T, Fujimura T, Fushida S, Kitagawa H, Kayahara M, Terada T and Miwa K. Trypsinogen expression in colorectal cancers. Int $\mathrm{J}$ Mol Med 6, 543-548 (2000).

Padlan EA. On the nature of antibody combining sites: unusual structural features that may confer on these sites an enhanced capacity for binding ligands. Proteins 7, 112-124 (1990).

Paju A, Hotakainen K, Cao, Y, Laurila T, Gadaleanu V, Hemminki A, Stenman $\mathrm{UH}$ and Bjartell A. Increased expression of tumor-associated trypsin inhibitor, TATI, in prostate cancer and in androgenindependent 22Rv1 cells. Eur Urol 52, 1670-1679 (2007).

Paju A and Stenman UH. Biochemistry and clinical role of trypsinogens and pancreatic secretory trypsin inhibitor. Crit Rev Clin. Lab Sci 43, 103-142 (2006).

Paju A, Vartiainen J, Haglund C, Itkonen O, von Boguslawski K, Leminen A, Wahlström, 
$\mathrm{T}$ and Stenman UH. Expression of trypsinogen-1, trypsinogen-2, and tumorassociated trypsin inhibitor in ovarian cancer: prognostic study on tissue and serum. Clin Cancer Res 10, 4761-4768 (2004).

Paju A, Jacobsen J, Rasmuson T, Stenman $\mathrm{UH}$ and Ljungberg B. Tumor associated trypsin inhibitor as a prognostic factor in renal cell carcinoma. J Urol 165, 959-962 (2001a).

Paju A, Sorsa T, Tervahartiala T, Koivunen E, Haglund C, Leminen A, Wahlstrom T, Salo T and Stenman UH. The levels of trypsinogen isoenzymes in ovarian tumour cyst fluids are associated with promatrix metalloproteinase-9 but not promatrix metalloproteinase-2 activation. Br J Cancer 84, 1363-1371 (2001b).

Paju A, Bjartell A, Zhang W M, Nordling S, Borgström, A, Hansson $J$ and Stenman UH. Expression and characterization of trypsinogen produced in the human male genital tract. Am J Pathol 157, 2011-2021 (2000).

Pasanen P, Eskelinen M, Kulju A, Penttilä I, Janatuinen E. and Alhava E. Tumourassociated trypsin inhibitor (TATI) in patients with colorectal cancer: a comparison with CEA, CA 50 and CA 242. Scand J Clin Lab Invest, 55, 119-124 (1995).

Pasternak A, Liu X, Lin TY and Hedstrom L. Activating a zymogen without proteolytic processing: mutation of Lys 15 and Asn 194 activates trypsinogen. Biochemistry 37, 16201-16210 (1998).

Patrinos GP, Kollia P, Loutradi-Anagnostou A, Loukopoulos D and Papadakis MN. The Cretan type of non-deletional hereditary persistence of fetal hemoglobin [A gamma$158 \mathrm{C}-->\mathrm{T}]$ results from two independent gene conversion events. Hum.Genet, 102, 629-634 (1998).

Perkal M, Marks C, Lorber M I. and Marks WH. A three-year experience with serum anodal trypsinogen as a biochemical marker for rejection in pancreatic allografts. False positives, tissue biopsy, comparison with other markers, and diagnostic strategies.
Transplantation 53, 415-419 (1992).

Perlmutter DH, Joslin G, Nelson P, Schasteen C, Adams SP and Fallon RJ. Endocytosis and degradation of alpha 1-antitrypsinprotease complexes is mediated by the serpin-enzyme complex (SEC) receptor. J Biol Chem 265, 16713-16716 (1990).

Perona JJ and Craik CS. Evolutionary divergence of substrate specificity within the chymotrypsin-like serine protease fold. J Biol Chem 272, 29987-29990 (1997).

Perona JJ, Hedstrom L, Rutter WJ and Fletterick RJ. Structural origins of substrate discrimination in trypsin and chymotrypsin. Biochemistry 34, 1489-1499 (1995).

Perona JJ, Tsu CA, Craik CS and Fletterick RJ. Crystal structures of rat anionic trypsin complexed with the protein inhibitors APPI and BPTI. J Mol Biol 230, 919-933 (1993).

Petersen $\mathrm{OH}$. Local and global $\mathrm{Ca}^{2+}$ signals: physiology and pathophysiology. Biol Res 37, 661-664 (2004).

Petersson U and BorgströmA. Characterization of immunoreactive trypsinogen activation peptide in urine in acute pancreatitis. JOP 7, 274-282 (2006).

Petersson U Appelros S and Borgström A. Different patterns in immunoreactive anionic and cationic trypsinogen in urine and serum in human acute pancreatitis. Int J Pancreatol 25, 165-170 (1999).

Pfützer R, Myers E, Applebaum-Shapiro S, Finch R, Ellis I, Neoptolemos J, Kant JA and Whitcomb DC. Novel cationic trypsinogen (PRSS1) N29T and R122C mutations cause autosomal dominant hereditary pancreatitis. Gut 50, 271-272 (2002).

Pfützer RH, Barmada MM, Brunskill AP, Finch R, Hart PS, Neoptolemos J, Furey WF and Whitcomb DC. SPINK1/PSTI polymorphisms act as disease modifiers in familial and idiopathic chronic pancreatitis. Gastroenterology 119, 615-623 (2000).

Piantino P and Arosaio E. Tumor-associated trypsin inhibitor, TATI, in gastrointestinal cancer and related benign diseases. Scand J Clin Lab Invest Suppl 207, 67-69 (1991). Pittman DD, Tomkinson KN, Michnick 
D, Selighsohn U and Kaufman RJ. Posttranslational sulfation of factor $\mathrm{V}$ is required for efficient thrombin cleavage and activation and for full procoagulant activity. Biochemistry 33, 6952-6959 (1994).

Pizzo SV. Serpin receptor 1: a hepatic receptor that mediates the clearance of antithrombin III-proteinase complexes. Am J Med 87, 10S-14S (1989).

Pratt CW, Church FC and Pizzo SV. In vivo catabolism of heparin cofactor II and its complex with thrombin: evidence for a common receptor-mediated clearance pathway for three serine proteinase inhibitors. Arch Biochem Biophys 262, 111-117 (1988).

Pratt CW, Roche PA and Pizzo SV. The role of inter-alpha-trypsin inhibitor and other proteinase inhibitors in the plasma clearance of neutrophil elastase and plasmin. Arch Biochem Biophys 258, 591-599 (1987).

Pratt CW and Pizzo SV. In vivo metabolism of inter-alpha-trypsin inhibitor and its proteinase complexes: evidence for proteinase transfer to alpha 2-macroglobulin and alpha 1-proteinase inhibitor. Arch Biochem Biophys 248, 587-596 (1986).

Prikk K, Maisi P, Sepper R, Stenman UH, Salo $\mathrm{T}$ and Sorsa T. Association of trypsin-2 with activation of gelatinase $B$ and collagenase-2 in human bronchoalveolar lavage fluid in vivo. Ann Med 33, 437-444 (2001).

Pubols MH, Bartelt DC and Greene LJ. Trypsin inhibitor from human pancreas and pancreatic juice. J Biol Chem 249, 2235-2242 (1974).

Rappsilber J, Steen H and Mann M. Labile sulfogroup allows differentiation of sulfotyrosine and phosphotyrosine in peptides. J Mass Spectrom 36, 832-833 (2001).

Raraty M, Ward J, Erdemli G, Vaillant C, Neoptolemos JP, Sutton R and Petersen OH. Calcium-dependent enzyme activation and vacuole formation in the apical granular region of pancreatic acinar cells. Proc Natl
Acad Sci USA 97, 13126-13131 (2000).

Richarz R, Tschesche $\mathrm{H}$ and Wuthrich $\mathrm{K}$. Carbon-13 nuclear magnetic resonance studies of the selectively isotopelabeled reactive site peptide bond of the basic pancreatic trypsin inhibitor in the complexes with trypsin, trypsinogen, and anhydrotrypsin. Biochemistry 19, 5711-5715 (1980).

Rinderknecht H. An elevated ratio of serum anionic to cationic trypsinogen may signal pancreatic disease. Int.J Pancreatol 20, 141 (1996).

RinderknechtH. Pancreatic secretory enzymes. In The Pancreas: biology, pathobiology and disease (V. L. W. Go, E. P. DiMagno JD. Gardner E. Lebenthal H. A Reger and G. A Scheele. eds.), 219-251, Raven Press, New York (1993).

Rinderknecht H, Adham NF, Renner IG and Carmack C. A possible zymogen selfdestruct mechanism preventing pancreatic autodigestion. Int J Pancreatol 3, 33-44 (1988).

Rinderknecht H. Activation of pancreatic zymogens. Normal activation, premature intrapancreatic activation, protective mechanisms against inappropriate activation. Dig Dis Sci 31, 314-321 (1986).

Rinderknecht H, Stace NH and Renner IG. Effects of chronic alcohol abuse on exocrine pancreatic secretion in man. Dig Dis Sci 30, 65-71 (1985).

Rinderknecht H, Renner IG, Abramson SB and Carmack C. Mesotrypsin: a new inhibitorresistant protease from a zymogen in human pancreatic tissue and fluid. Gastroenterology 86, 681-692 (1984).

Rinderknecht H, Renner IG and Carmack C. Trypsinogen variants in pancreatic juice of healthy volunteers, chronic alcoholics, and patients with pancreatitis and cancer of the pancreas. Gut, 20, 886-891 (1979).

Rinderknecht $\mathrm{H}$ and Geokas MC. Anionic and cationic trypsinogens (trypsins) in mammalian pancreas. Enzyme 14, 116-130 (1972).

Riordan JR, Rommens JM, Kerem B, Alon N, Rozmahel R, Grzelczak Z, Zielenski J, Lok 
$\mathrm{S}$, Plavsic $\mathrm{N}$ and Chou JL. Identification of the cystic fibrosis gene: cloning and characterization of complementary DNA. Science 245, 1066-1073 (1989).

Roach JC, Wang K, Gan L and Hood L. The molecular evolution of the vertebrate trypsinogens. J Mol Evol 45, 640-652 (1997).

Roberts RM, Mathialagan N, Duffy JY and Smith GW. Regulation and regulatory role of proteinase inhibitors. Crit Rev Eukaryot Gene Expr 5, 385-436 (1995).

Robinson NC, Neurath H and Walsh KA. The relation of the $\alpha$-amino group of trypsin to enzyme function and zymogen activation. Biochemistry 12, 420-426 (1973).

Rock MJ, Hoffman G, Laessig RH, Kopish GJ, Litsheim TJ and Farrell PM. Newborn screening for cystic fibrosis in Wisconsin: nine-year experience with routine trypsinogen/DNA testing. J Pediatr 147, S73-7 (2005).

Rose SD, Swift GH, Peyton MJ, Hammer RE and MacDonald RJ. The role of PTF1-P48 in pancreatic acinar gene expression. $\mathrm{J}$ Biol Chem 276, 44018-44026 (2001).

Rosewicz S, Lewis LD, Wang XY, Liddle R. $A$ and Logsdon CD. Pancreatic digestive enzyme gene expression: effects of CCK and soybean trypsin inhibitor. Am J Physiol 256, G733-8 (1989).

Roux E, Strubin M, Hagenbuchle $O$ and WellauerPK. The cell-specific transcription factor PTF1 contains two different subunits that interact with the DNA. Genes Dev, 3, 1613-1624 (1989).

Rowen L, Williams E, Glusman G, Linardopoulou E, Friedman C, Ahearn ME, Seto J, Boysen C, Qin S, Wang K, Kaur A, Bloom S, Hood L and Trask BJ. Interchromosomal segmental duplications explain the unusual structure of PRSS3, the gene for an inhibitor-resistant trypsinogen. Mol Biol Evol 22, 1712-1720 (2005).

Rowen L, Koop BF and Hood L. The complete 685-kilobase DNA sequence of the human beta $\mathrm{T}$ cell receptor locus. Science 272, 1755-1762 (1996).

Rühlmann A, Kukla D, Schwager P, Bartels K and Huber R. Structure of the complex formed by bovine trypsin and bovine pancreatic trypsin inhibitor. Crystal structure determination and stereochemistry of the contact region. J Mol Biol 77, 417-436 (1973).

Rydel TJ, Tulinsky A, Bode W and Huber R. Refined structure of the hirudin-thrombin complex. J Mol Biol 221, 583-601 (1991).

Rydel TJ, Ravichandran KG, Tulinsky A, Bode W, Huber R, Roitsch C and Fenton JW 2nd. The structure of a complex of recombinant hirudin and human alphathrombin. Science 249, 277-280 (1990).

Rypniewski WR, Perrakis A, Vorgias CE and Wilson KS. Evolutionary divergence and conservation of trypsin. Protein Eng 7, 57-64 (1994).

Saharinen P, Kerkelä K, Ekman N, Marron M, Brindle N, Lee GM, Augustin H, Koh, GY and Alitalo K. Multiple angiopoietin recombinant proteins activate the Tie1 receptor tyrosine kinase and promote its interaction with Tie2. J Cell Biol 169, 239-243 (2005).

Sahin-Tóth M. Human mesotrypsin defies natural trypsin inhibitors: from passive resistance to active destruction. Protein Pept Lett, 12, 457-464 (2005).

Sahin-Tóth M. The pathobiochemistry of hereditary pancreatitis: studies on recombinant human cationic trypsinogen. Pancreatology, 1, 461-465 (2001).

Sahin-Tóth M. Human cationic trypsinogen. Role of Asn-21 in zymogen activation and implications in hereditary pancreatitis. J Biol Chem 275, 22750-22755 (2000).

Sahin-Tóth M and Tóth M. Gain-of-function mutations associated with hereditary pancreatitis enhance autoactivation of human cationic trypsinogen. Biochem Biophys Res Commun 278, 286-289 (2000).

Sahin-Tóth M, Kukor Z and Nemoda Z. Human cationic trypsinogen is sulfated on Tyr154. FEBS Journal 273, 5044-5050 (2006).

Sainio V, Puolakkainen P, Kemppainen E, Hedström, J, Haapiainen R, Kivisaari L, Stenman UH, Schröder T and Kivilaakso 
E. Serum trypsinogen-2 in the prediction of outcome in acute necrotizing pancreatitis. Scand J Gastroenterol 31, 818-824 (1996).

Salier JP, Rouet P, Raguenez G and Daveau M. The inter-alpha-inhibitor family: from structure to regulation. Biochem J 315, 1-9 (1996).

Salier JP. Inter-alpha-trypsin inhibitor: emergence of a family within the Kunitztype protease inhibitor superfamily. Trends Biochem Sci 15, 435-439 (1990).

Saluja AK, Donovan EA, Yamanaka K, Yamaguchi Y, Hofbauer B and Steer ML. Cerulein-induced in vitro activation of trypsinogen in rat pancreatic acini is mediated by cathepsin B. Gastroenterology 113, 304-310 (1997).

Sankaralingam S, Wesen C, Barawi M, Galera R and Lloyd L. Use of the urinary trypsinogen-2 dip stick test in early diagnosis of pancreatitis after endoscopic retrograde cholangiopancreatography. Surg Endosc 21, 1312-1315 (2007).

Sans MD, Tashiro M, Vogel NL, Kimball SR, D'Alecy LG and Williams JA. Leucine activates pancreatic translational machinery in rats and mice through mTOR independently of CCK and insulin. J Nutr, 136, 1792-1799 (2006).

Sardiello, M, Annunziata I, Roma G and Ballabio A. Sulfatases and sulfatase modifying factors: an exclusive and promiscuous relationship. Hum.Mol Genet, 14, 3203-3217 (2005).

Sasaki T, Larsson H, Kreuger J, Salmivirta M, Claesson-Welsh, L, Lindahl U, Hohenester E and Timpl R. Structural basis and potential role of heparin/heparan sulfate binding to the angiogenesis inhibitor endostatin. EMBO J 18, 6240-6248 (1999).

Schechter I and Berger A. On the size of the active site in proteases. I. Papain. Biochem Biophys Res Commun 27, 157-162 (1967).

Scheele G, Bartelt D and Bieger W. Characterization of human exocrine pancreatic proteins by two-dimensional isoelectric focusing/sodium dodecyl sulfate gel electrophoresis. Gastroenterology 80, 461-473 (1981).

Schellenberger V, Turck, CW and Rutter WJ. Role of the S' subsites in serine protease catalysis. Active-site mapping of rat chymotrypsin, rat trypsin, alpha-lytic protease, and cercarial protease from Schistosoma mansoni. Biochemistry 33, 4251-4257 (1994).

Schneider A, Suman A, Rossi L, Barmada MM, Beglinger C, Parvin S, Sattar S, Ali L, Khan A K, Gyr N and Whitcomb, DC. SPINK1/PSTI mutations are associated with tropical pancreatitis and type II diabetes mellitus in Bangladesh. Gastroenterology 123, 1026-1030 (2002).

Schneider SL and Laskowski M Sr. Occurrence of two cleavages preceding inactivation of bovine temporary trypsin isoinhibitor A. J Biol Chem 249, 2009-2015 (1974).

Schneider SL, Stasiuk L and Laskowski M Sr. Sequence of tryptic cleavages in porcine pancreatic secretory inhibitor II. J Biol Chem 248, 7207-7214 (1973).

Schwiebert EM, Benos DJ and Fuller CM. Cystic fibrosis: a multiple exocrinopathy caused by dysfunctions in a multifunctional transport protein. Am J Med 104, 576-590 (1998).

Scott G, Leopardi S, Printup S, Malhi N, Seiberg, M and Lapoint, R. Proteinaseactivated receptor-2 stimulates prostaglandin production in keratinocytes: analysis of prostaglandin receptors on human melanocytes and effects of PGE2 and PGF2alpha on melanocyte dendricity. J Invest Dermatol 122, 1214-1224 (2004). Sharer N, Schwarz, M, Malone G, Howarth, A, Painter J, Super M and Braganza J. Mutations of the cystic fibrosis gene in patients with chronic pancreatitis. N Engl J Med 339, 645-652 (1998).

Shibata T, Ogawa M, Matsuda K, Miyauchi K, Yamamoto T and Mori T. Purification and characterization of pancreatic secretory trypsin inhibitor in human gastric mucosa. Clin Chim Acta 159, 27-36 (1986).

Shumaker H, Amlal H, Frizzell R, Ulrich CD 2nd and Soleimani M. CFTR drives $\mathrm{Na}^{+}-$ $n \mathrm{HCO}_{3}^{-}$cotransport in pancreatic duct 
cells: a basis for defective $\mathrm{HCO}_{3}^{-}$secretion in CF. Am J Physiol 276, 16-25 (1999).

Sichler K, Hopfner KP, Kopetzki E, Huber R, Bode $\mathrm{W}$ and Brandstetter $\mathrm{H}$. The influence of residue 190 in the $\mathrm{S} 1$ site of trypsin-like serine proteases on substrate selectivity is universally conserved. FEBS Lett 530, 220-224 (2002).

Silverman GA, Bird PI, Carrell RW, Church FC, Coughlin PB, Gettins PG, Irving JA, Lomas DA, Luke CJ, Moyer RW, Pemberton PA, Remold-O'Donnell E, Salvesen GS, Travis J and Whisstock JC. The serpins are an expanding superfamily of structurally similar but functionally diverse proteins. Evolution, mechanism of inhibition, novel functions, and a revised nomenclature. J Biol Chem 276, 33293-33296 (2001).

Simon P, Weiss FU, Sahin-Tóth M, Parry M, Nayler O, Lenfers B, Schnekenburger J, Mayerle J, Domschke W and Lerch MM. Hereditary pancreatitis caused by a novel PRSS1 mutation (Arg-122 --> Cys) that alters autoactivation and autodegradation of cationic trypsinogen. J Biol Chem 277, 5404-5410 (2002).

Singh $J$ and Thornton JM. SIRIUS. An automated method for the analysis of the preferred packing arrangements between protein groups. J Mol Biol 211, 595-615 (1990).

Soini E, Hemmilä I and Dahlen P. Timeresolved fluorescence in biospecific assays. Ann.Biol Clin.(Paris), 48, 567-571 (1990).

Soini E and Kojola H. Time-resolved fluorometer for lanthanide chelates--a new generation of nonisotopic immunoassays. Clin Chem 29, 65-68 (1983).

Sommer L, Hagenbuchle O, Wellauer PK and Strubin M. Nuclear targeting of the transcription factor PTF1 is mediated by a protein subunit that does not bind to the PTF1 cognate sequence. Cell 67, 987-994 (1991).

Sorsa T, Salo, T, Koivunen E, Tyynelä J, Konttinen YT, Bergmann U, Tuuttila A, Niemi E, Teronen O, Heikkilä P, Tschesche H, Leinonen J, Osman S. and Stenman UH.
Activation of type IV procollagenases by human tumor-associated trypsin-2. J Biol Chem 272, 21067-21074 (1997).

Sottrup-Jensen L. Alpha-macroglobulins: structure, shape, and mechanism of proteinase complex formation. J Biol Chem 264, 11539-11542 (1989).

Spence WC, Paulus-Thomas J, Orenstein DM and Naylor EW. Neonatal screening for cystic fibrosis: addition of molecular diagnostics to increase specificity. Biochem Med Metab Biol 49, 200-211 (1993).

Sprang S, Standing T, Fletterick, R.J, Stroud RM, Finer-Moore J, Xuong NH, Hamlin R, Rutter WJ and Craik CS. The threedimensional structure of Asn 102 mutant of trypsin: role of Asp102 in serine protease catalysis. Science 237, 905-909 (1987).

Stavridi ES, O’Malley K, Lukacs CM, Moore WT, Lambris JD, Christianson DW, Rubin $\mathrm{H}$ and Cooperman BS. Structural change in alpha-chymotrypsin induced by complexation with alpha 1-antichymotrypsin as seen by enhanced sensitivity to proteolysis. Biochemistry 35, 10608-10615 (1996).

Steinbuch $\mathrm{M}$ and Loeb, J. Isolation of an alpha2-globulin from human plasma. Nature 192, 1196 (1961).

Steinhoff M, Buddenkotte J, Shpacovitch V, Rattenholl A, Moormann C, Vergnolle $\mathrm{N}$, Luger TA and Hollenberg MD. Proteinase-activated receptors: transducers of proteinase-mediated signaling in inflammation and immune response. Endocr.Rev, 26, 1-43 (2005).

Stenman M, Ainola M, Valmu L, Bjartell A, Ma G, Stenman UH, Sorsa T, Luukkainen $\mathrm{R}$ and Konttinen YT. Trypsin-2 degrades human type II collagen and is expressed and activated in mesenchymally transformed rheumatoid arthritis synovitis tissue. Am J Pathol 167, 1119-1124 (2005).

Stenman M, Paju A, Hanemaaijer R, Tervahartiala T, Leminen A, Stenman UH, Konttinen YT and Sorsa T. Collagenases (MMP-1, -8 and -13) and trypsinogen-2 in fluid from benign and malignant ovarian cysts. Tumour Biol 24, 9-12 (2003).

Stenman UH, Koivunen E and Itkonen O. 
Biology and function of tumor-associated trypsin inhibitor, TATI. Scand J Clin Lab Invest Suppl 207, 5-9 (1991).

Stenman UH, Huhtala ML, Koistinen $\mathrm{R}$ and Seppälä M. Immunochemical demonstration of an ovarian cancerassociated urinary peptide. Int $\mathrm{J}$ Cancer 30, 53-57 (1982).

Stone SR. and Hofsteenge J. Kinetics of the inhibition of thrombin by hirudin. Biochemistry 25, 4622-4628 (1986).

Stroud RM, Kay L. M and Dickerson RE. The structure of bovine trypsin: electron density maps of the inhibited enzyme at 5 Ångstrom and at 2-7 Ångstrom resolution. J Mol Biol 83, 185-208 (1974).

Sweet RM, Wright HT, Janin J, Chothia CH and Blow DM. Crystal structure of the complex of porcine trypsin with soybean trypsin inhibitor (Kunitz) at 2.6- $\AA$ resolution. Biochemistry 13, 4212-4228 (1974).

Szepessy E. and Sahin-Tóth M. Human mesotrypsin exhibits restricted S1' subsite specificity with a strong preference for small polar side chains. FEBS J 273, 2942-2954 (2006).

Szilagyi L, Kenesi E, Katona G, Kaslik, G, Juhasz $\mathrm{G}$ and Graf L. Comparative in vitro studies on native and recombinant human cationic trypsins. Cathepsin B is a possible pathological activator of trypsinogen in pancreatitis. J Biol Chem 276, 24574-24580 (2001).

Szmola R. and Sahin-Tóth M. Chymotrypsin $\mathrm{C}$ (caldecrin) promotes degradation of human cationic trypsin: identity with Rinderknecht's enzyme Y. Proc Natl Acad Sci USA 104, 11227-11232 (2007).

Szmola R, Kukor Z and Sahin-Tóth M. Human mesotrypsin is a unique digestive protease specialized for the degradation of trypsin inhibitors. J Biol Chem 278, 48580-48589 (2003).

Talamini G, Bassi C, Falconi M Frulloni L, Di Francesco, V, Vaona B, Bovo, P, Rigo, L, Castagnini A, Angelini G, Vantini I, Pederzoli $\mathrm{P}$ and Cavallini G. Cigarette smoking: an independent risk factor in alcoholic pancreatitis. Pancreas 12,
131-137 (1996).

Tallan HH, Bella ST, Stein WH. and Moore $\mathrm{S}$. Tyrosine- $O$-sulfate as a constituent of normal human urine. J Biol Chem 217, 703-708 (1955).

Tani T, Kawashima I Mita K and Takiguchi Y. Nucleotide sequence of the human pancreatic trypsinogen III cDNA. Nucleic Acids Res 18, 1631 (1990).

Teich N, Le Maréchal C, Kukor, Z, Caca K, Witzigmann H, Chen JM Tóth, M Mossner J, Keim V, Feréc C. and Sahin-Tóth M. Interaction between trypsinogen isoforms in genetically determined pancreatitis: mutation E79K in cationic trypsin (PRSS1) causes increased transactivation of anionic trypsinogen (PRSS2). Hum Mutat 23, 22-31 (2004).

Teich N, Bodeker H and Keim V. Cathepsin $\mathrm{B}$ cleavage of the trypsinogen activation peptide. BMC Gastroenterol 2, 16 (2002).

Teich N, Ockenga J, Hoffmeister A, Manns M Mossner $\mathrm{J}$ and Keim V. Chronic pancreatitis associated with an activation peptide mutation that facilitates trypsin activation. Gastroenterology 119, 461-465 (2000).

Tenner S, Fernandez-del Castillo, C, Warshaw, A, Steinberg W, Hermon-Taylor J, Valenzuela JE, Hariri M, Hughes M and Banks PA. Urinary trypsinogen activation peptide (TAP) predicts severity in patients with acute pancreatitis. Int $\mathrm{J}$ Pancreatol 21, 105-110 (1997).

Terada T, Kitamura Y, Ashida K, Matsunaga Y, Kato, M, Harada K, Morita T, Ohta $\mathrm{T}$ and Nakanuma $\mathrm{Y}$. Expression of pancreatic digestive enzymes in normal and pathologic epithelial cells of the human gastrointestinal system. Virchows Arch 431, 195-203 (1997).

Terada T, Ohta T, Minato H and Nakanuma Y. Expression of pancreatic trypsinogen/ trypsin and cathepsin B in human cholangiocarcinomas and hepatocellular carcinomas. Hum Pathol 26, 746-752 (1995).

Tomita N, Horii A, Yamamoto T, Ogawa M, Mori T. and Matsubara K. Expression of pancreatic secretory trypsin inhibitor gene 
in neoplastic tissues. FEBS Lett, 225, 113-119 (1987).

Tooze J, Hollinshead M, Hensel G, Kern HF and Hoflack B. Regulated secretion of mature cathepsin B from rat exocrine pancreatic cells. Eur J Cell Biol 56, 187-200 (1991).

Tramonti G, Ferdeghini M, Annichiarico, C, Donadio C, Norpoth M, Mantuano $\mathrm{E}$ and Bianchi C. Assessment of tumorassociated trypsin inhibitor (TATI) as a marker of renal function. J nephrol 16, 663-672 (2003).

Travis $\mathrm{J}$ and Salvesen GS. Human plasma proteinase inhibitors. Annu Rev Biochem, 52, 655-709 (1983).

Travis $J$ and Roberts RC. Human trypsin. Isolation and physical-chemical characterization. Biochemistry 8, 2884-2889 (1969).

Turvey MR, Fogarty KE and Thorn P. Inositol $(1,4,5)$-trisphosphate receptor links to filamentous actin are important for generating local $\mathrm{Ca}^{2+}$ signals in pancreatic acinar cells. J Cell Sci 118, 971-980 (2005).

Umenishi F, Yasumitsu H, Ashida Y, Yamauti J, Umeda M and Miyazaki K. Purification and properties of extracellular matrix-degrading metallo-proteinase overproduced by Rous sarcoma virustransformed rat liver cell line, and its identification as transin. $\mathrm{J}$ Biochem. (Tokyo), 108, 537-543 (1990).

Vader G, Medema RH and Lens SM. The chromosomal passenger complex: guiding Aurora-B through mitosis. J Cell Biol 173, 833-837 (2006).

Van Acker GJ, Saluja AK, Bhagat L, Singh VP, Song AM and Steer ML. Cathepsin B inhibition prevents trypsinogen activation and reduces pancreatitis severity. Am J Physiol Gastrointest Liver Physiol 283, G794-800 (2002).

van der Horst A and Burgering BM. Stressing the role of FoxO proteins in lifespan and disease. Nat Rev Mol Cell Biol 8, 440-450 (2007).

Varallyay E, Lengyel Z, Graf L and Szilagyi L. The role of disulfide bond C191-C220 in trypsin and chymotrypsin. Biochem Biophys Res Commun 230, 592-596 (1997).

Vargas F, Frerot, O, Dan Tung Tuong M and Schwartz JC. Characterization of a tyrosine sulfotransferase in rat brain using cholecystokinin derivatives as acceptors. Biochemistry 24, 5938-5943 (1985).

Venesmaa P, Stenman UH, Forss M, Leminen A, Lehtovirta P, Vartiainen J and Paavonen J. Pre-operative serum level of tumourassociated trypsin inhibitor and residual tumour size as prognostic indicators in Stage III epithelial ovarian cancer. Br J Obstet Gynaecol 105, 508-511 (1998).

Venesmaa P, Lehtovirta P, Stenman UH, Leminen A, Forss $\mathrm{M}$ and Ylikorkala O. Tumour-associated trypsin inhibitor (TATI): comparison with CA125 as a preoperative prognostic indicator in advanced ovarian cancer. Br J Cancer 70, 1188-1190 (1994).

Vercaigne-Marko, D, Carrere J, Guy-Crotte $\mathrm{O}$, Figarella $\mathrm{C}$ and Hayem A. Human cationic and anionic trypsins: differences of interaction with alpha 1-proteinase inhibitor. Biol Chem Hoppe Seyler 370, 1163-1171 (1989).

Vergnolle N, Ferazzini M, D’Andrea MR, Buddenkotte $\mathrm{J}$ and Steinhoff $\mathrm{M}$. Proteinase-activated receptors: novel signals for peripheral nerves. Trends Neurosci 26, 496-500 (2003).

Vergote IB, Kaern J, Abeler VM, Pettersen EO, De Vos LN and Trope CG. Analysis of prognostic factors in stage I epithelial ovarian carcinoma: importance of degree of differentiation and deoxyribonucleic acid ploidy in predicting relapse. Am J Obstet Gynecol 169, 40-52 (1993).

Vervoorts J, Luscher-Firzlaff $\mathrm{J}$ and Luscher B. The ins and outs of MYC regulation by posttranslational mechanisms. J Biol Chem 281, 34725-34729 (2006).

Vukicevic, M, Weder G, Boillat, A, Boesch A and Kellenberger S. Trypsin cleaves acidsensing ion channel 1a in a domain that is critical for channel gating. J Biol Chem 281, 714-722 (2006).

Wachter E and Hochstrasser K. Kunitz-type 
proteinase inhibitors derived by limited proteolysis of the inter-alpha-trypsin inhibitor, IV. The amino acid sequence of the human urinary trypsin inhibitor isolated by affinity chromatography. Hoppe Seylers Z.Physiol.Chem, 362, 1351-1355 (1981).

Walker MD, Edlund T, Boulet, AM and Rutter WJ. Cell-specific expression controlled by the 5'-flanking region of insulin and chymotrypsin genes. Nature 306, 557-561 (1983).

Wang WQ, Sun JP and Zhang ZY. An overview of the protein tyrosine phosphatase superfamily. Curr Top Med Chem, 3, 739-748 (2003).

Wang Y, Luo, W, Wartmann T, Halangk W, Sahin-Tóth M and Reiser G. Mesotrypsin, a brain trypsin, activates selectively proteinase-activated receptor-1, but not proteinase-activated receptor-2, in rat astrocytes. J Neurochem, 99, 759-769 (2006).

Wasle B and Edwardson JM. The regulation of exocytosis in the pancreatic acinar cell. Cell Signal 14, 191-197 (2002).

Weber PC, Lee SL Lewandowski FA, Schadt $\mathrm{MC}$, Chang $\mathrm{CH}$ and Kettner CA. Kinetic and crystallographic studies of thrombin with Ac-(D)Phe-Pro-boroArg-OH and its lysine, amidine, homolysine, and ornithine analogs. Biochemistry 34, 3750-3757 (1995).

Weiner SJ, Seibel GL and Kollman PA. The nature of enzyme catalysis in trypsin. Proc Natl Acad Sci USA 83, 649-653 (1986).

Whitcomb D. C. Early trypsinogen activation in acute pancreatitis. Gastroenterology 116, 770-772 (1999).

Whitcomb DC, Gorry MC, Preston RA, Furey W, Sossenheimer MJ, Ulrich CD, Martin SP, Gates LK Jr, Amann ST, Toskes PP, Liddle R, McGrath K, Uomo G, Post JC and Ehrlich GD. Hereditary pancreatitis is caused by a mutation in the cationic trypsinogen gene. Nat Genet, 14, 141-145 (1996).

Wiegand U, Corbach S, Minn A, Kang J and Muller-Hill B. Cloning of the cDNA encoding human brain trypsinogen and characterization of its product. Gene 136,
167-175 (1993).

Wiksten JP, Lundin J, Nordling S, Kokkola A, Stenman UH and Haglund C. High tissue expression of tumour-associated trypsin inhibitor (TATI) associates with a more favourable prognosis in gastric cancer. Histopathology, 46, 380-388 (2005).

Wilkins PP, Moore KL, McEver RP and Cummings RD. Tyrosine sulfation of $\mathrm{P}$-selectin glycoprotein ligand-1 is required for high affinity binding to P-selectin. J Biol Chem 270, 22677-22680 (1995).

William S, Ramaprasad P and Kasinathan C. Purification of tyrosylprotein sulfotransferase from rat submandibular salivary glands. Arch Biochem Biophys 338, 90-96 (1997).

Williams JA. Regulation of pancreatic acinar cell function. Curr Opin Gastroenterol 22, 498-504 (2006).

Williams JA. Intracellular signaling mechanisms activated by cholecystokininregulating synthesis and secretion of digestive enzymes in pancreatic acinar cells. Annu Rev Physiol 63, 77-97 (2001).

Williams SJ Gotley DC and Antalis TM. Human trypsinogen in colorectal cancer. Int J Cancer 93, 67-73 (2001).

Witt H, Sahin-Tóth M, Landt $\mathrm{O}$ et al. A degradation-sensitive anionic trypsinogen (PRSS2) variant protects against chronic pancreatitis. Nat Genet 38, 668-673 (2006).

Witt H, Luck W, Hennies HC, Classen M, Kage A, Lass U, Landt $\mathrm{O}$ and Becker M. Mutations in the gene encoding the serine protease inhibitor, Kazal type 1 are associated with chronic pancreatitis. Nat Genet, 25, 213-216 (2000).

Woods A S, Wang HY and Jackson SN. Sulfation, the up-and-coming posttranslational modification: its role and mechanism in protein-protein interaction. J Proteome Res 6, 1176-1182 (2007).

Wu J, Liu F, Nilsson A and Duan RD. Pancreatic trypsin cleaves intestinal alkaline sphingomyelinase from mucosa and enhances the sphingomyelinase activity. Am J Physiol Gastrointest Liver Physiol 287, G967-73 (2004). 
Yamada H, Le QT, Kousaka A, Higashi Y, Tsukane $M$ and Kido $H$. Sendai virus infection up-regulates trypsin I and matrix metalloproteinase-9, triggering viral multiplication and matrix degradation in rat lungs and lung L2 cells. Arch Virol 151, 2529-2537 (2006).

Yamamoto H, Iku S, Adachi Y, Imsumran A, Taniguchi H, Nosho, K, Min Y, Horiuchi S, Yoshida M, Itoh, F and Imai K. Association of trypsin expression with tumour progression and matrilysin expression in human colorectal cancer. $\mathrm{J}$ Pathol 199, 176-184 (2003).

Yamamoto H, Iku S, Itoh F, Tang X, Hosokawa $\mathrm{M}$ and Imai $\mathrm{K}$. Association of trypsin expression with recurrence and poor prognosis in human esophageal squamous cell carcinoma. Cancer 91, 1324-1331 (2001).

Yamashita K, Mimori K, Inoue H, Mori M and Sidransky D. A tumor-suppressive role for trypsin in human cancer progression. Cancer Res 63, 6575-6578 (2003).

Yang K, Ding YX and Chin WC. $\mathrm{K}^{+}$-induced ion-exchanges trigger trypsin activation in pancreas acinar zymogen granules. Arch. Biochem Biophys 459, 256-263 (2007).
Yasuda T, Yasuda T, Ohmachi Y, Katsuki M, Yokoyama M, Murata A, Monden M and Matsubara K. Identification of novel pancreas-specific regulatory sequences in the promoter region of human pancreatic secretory trypsin inhibitor gene. J Biol Chem 273, 34413-34421 (1998).

Yasuda T, Ogawa M, Murata A, Ohmachi Y, Yasuda T, Mori T and Matsubara K. Identification of the IL-6-responsive element in an acute-phase-responsive human pancreatic secretory trypsin inhibitor-encoding gene. Gene 131, 275-280 (1993).

Yasuda T, Ogawa M, Murata A, Oka Y, Uda K and Mori T. Response to IL-6 stimulation of human hepatoblastoma cells: production of pancreatic secretory trypsin inhibitor. Biol Chem Hoppe Seyler 371, 95-100 (1990).

Zamolodchikova TS, Sokolova EA, Lu D and Sadler JE. Activation of recombinant proenteropeptidase by duodenase. FEBS Lett 466, 295-299 (2000).

Zhou H, Watts JD and Aebersold R. A systematic approach to the analysis of protein phosphorylation. Nat Biotechnol 19, 375-378 (2001). 